\title{
DIFFUSIVE LIMIT OF LATTICE GAS WITH MIXING CONDITIONS*
}

\author{
S.R.S. VARADHAN ${ }^{\dagger}$ AND HORNG-TZER YAU ${ }^{\ddagger}$
}

\begin{abstract}
We prove, under certain mixing conditions, that the hydrodynamical limit of a stochastic lattice gas on the cubic lattice $\mathbb{Z}^{d}$ is governed by a nonlinear diffusion equation. Following [V1], we characterize the diffusion coefficient by a variational formula, which is equivalent to the Green-Kubo formula. The fluctuation-dissipation equation is established rigorously as an important step of the proof. Our mixing conditions are implied by the Dobrushin-Shlosman mixing conditions which are always valid at high temperatures.
\end{abstract}

1. Introduction. Classical or Newtonian mechanics is described by a system of ordinary differential equations involving usually a two-body interaction potential. When the number of particles involved is large, rather than describe the motion of every particle, one usually tries to describe the collective behavior of these particles. The typical formulation involves the consideration of local averages of conserved quantities like density, average velocity, and energy. Since these local averages of conserved quantities tend to vary slowly in time, under some suitable space-time rescaling, referred to as hydrodynamical scaling, we expect to obtain a closed system of equations for these conserved quantities, as functions of macroscopic space and time. While such a transition is reasonably well understood from a physical point of view, these problems appear to be far beyond the current available methods for a rigorous mathematical treatment.

One way to make the problem tractable is to introduce some noise or randomness into the dynamics and in this way we obtain stochastic interacting particle systems. There are many interesting models that have been studied successfully, and we wish to mention two of them. The first one is a Hamiltonian system perturbed by a weak noise considered in [OVY], that leads to the Euler equation under hydrodynamical scaling. The scaling there is the hyperbolic scaling $x \rightarrow \varepsilon x$ and $t \rightarrow \varepsilon^{-1} t$. The second model is one of interacting Brownian motions. Here the dynamics is controlled by the infinitesimal generator for the Markovian motion of these interacting Brownian particles. The infinitesimal generator involves a second order term which is the Laplace operator defining the noise, perturbed by a drift term or a first order term that comes from two particle interactions like in the Hamiltonian case. The similarity extends further in that the invariant measures are Gibbs measures very much like in the Hamiltonian case.

The natural scaling here is however the diffusive scaling $x \rightarrow \varepsilon x, t \rightarrow \varepsilon^{-2} t$ and the limiting equation is expected to be a nonlinear diffusion equation. This is rigorously

*Received September 26, 1997; accepted for publication November 19, 1997.

†Courant Institute of Mathematical Sciences, New York University, 251 Mercer Street, New York, NY 10012, USA (varadhan@cims.nyu.edu). Research partially supported by U. S. National Science Foundation grant NSF-DMS-9503419 and Army Research Office grant ARO-DAAH04-95-10666 .

$\ddagger$ Courant Institute of Mathematical Sciences, New York University, 251 Mercer Street, New York, NY 10012, USA (yau@math.nyu.edu). Research partially supported by U. S. National Science Foundation grants DMS-9403462 and DMS-9703752, as well as by a David and Lucile Packard Foundation Fellowship. 
established in [V2] for dimension $d=1$. For dimension $d \geq 2$ and away from phase transition, one can prove it by applying the relative entropy argument of [Y1].

If we replace the Brownian motions by random walks, we obtain lattice gas models. The invariant measures are thus Gibbs states on $\mathbb{Z}^{d}$. One would expect that the hydrodynamical limit should be much easier to establish for lattice gases than for interacting Brownian motions. This turns out to be wrong. The main reason is that while the interacting Brownian motions model satisfies the 'gradient condition', lattice gas models in general do not. We will now explain briefly what the gradient condition means and why it is important in establishing hydrodynamical limits under diffusive scaling. To keep the notation simple let us take $d=1$.

Denote by $\eta=\left(\eta_{x}\right)_{x \in \mathbb{Z}}$ with $\eta_{x} \in\{0,1\}$ a typical configuration of of particles in $\mathbb{Z}$ with $\eta_{x}$ denoting the number of particles at $x$. From the dynamics of our lattice gas model, we can write down the microscopic conservation law

$$
d \eta_{x}=\left[w_{x-1, x}-w_{x, x+1}\right] d t+d M_{x}(t)
$$

where $w_{x, x+1}$ is the current along the bond $(x, x+1)$ and $d M_{x}$ is a martingale term due to the noise. Because our goal is to derive a diffusive limit, it is most convenient if the current is itself a gradient. In other words, $w_{x, x+1}(\eta)=h\left(\tau_{x} \eta\right)-h\left(\tau_{x+1} \eta\right)$ for some local function $h$; here $\tau_{x}$ is the shift by $x$. This is the condition that is called the 'gradient condition'[KLS]. An analogous condition is satisfied in the interacting Brownian motions model but the condition is in general not satisfied for lattice gas models. Let us take a quick look at the role played by the 'gradient condition'. If we want to study the change of density as a function of space and time under diffusive scaling we need to study

$$
d \frac{1}{N} \sum_{x} J\left(\frac{x}{N}\right) \eta_{x}\left(N^{2} t\right)=\frac{N}{N} \sum_{x} J^{\prime}\left(\frac{x}{N}\right) w_{x, x+1}(\eta) d t+d M_{N}(t)
$$

where $J$ is a test function. If the 'gradient condition' holds we can simplify (1.1) to get

$$
d \frac{1}{N} \sum_{x} J\left(\frac{x}{N}\right) \eta_{x}\left(N^{2} t\right) \simeq \frac{1}{N} \sum_{x} J^{\prime \prime}\left(\frac{x}{N}\right) h\left(\tau_{x} \eta\right) d t+d M_{N}(t)
$$

If we believe in the validity of a local averaging principle, then the term $h\left(\tau_{x} \eta\right)$ can be replaced by $\hat{h}(\rho(t, x))$, where $\hat{h}(\rho)$ is the expected value of $h(\cdot)$ in the Gibbs state with density $\rho$. The martingale term becomes negligible for large $N$ and we end up with the nonlinear diffusion equation

$$
\frac{\partial \rho}{\partial t}=[h(\rho(t, x))]_{x x}=\left[h^{\prime}(\rho(t, x)) \rho_{x}(t, x)\right]_{x}
$$

It is not clear how we would ever arrive at an equation like (1.3) without the gradient condition.

One way to bypass the gradient condition is to obtain a fluctuation-dissipation equation. A difficult step for us will be to establish the relation

$$
w_{0,1}(\eta)=D(\rho)\left(\eta_{0}-\eta_{1}\right)+\mathcal{L} g
$$

where $\mathcal{L}$ is the generator of the dynamics and $\mathcal{L} g$ represents fluctuation in a certain sense. One then obtains a diffusive limit

$$
\frac{\partial \rho}{\partial t}=\left[D(\rho(t, x)) \rho_{x}(t, x)\right]_{x}
$$


This step, while unnecessary for gradient models, is the key step for nongradient models.

A curious fact concerning lattice gases is the difficulty of constructing gradient models [S]. Except when the dimension $d$ is 1 or when the Gibbs measures degenerate into product measures, we are not aware of any gradient lattice gas models. If, instead of the lattice gas models, we consider Ginzburg-Laudau models, there are plenty of examples satisfying the gradient condition. The hydrodynamical limit in this case is established in [R], following the approach of [GPV], and is valid even in the phase transition region. On the other hand, one can easily construct nongradient versions of Ginzburg-Laudau models or interacting Brownian motions. Indeed, the hydrodynamical limit for a nongradient Ginzburg-Landau model at infinite temperature is established by [V1] where a basic outline for the analysis of nongradient models is first given. It is then extended to lattice gases at infinite temperature in [Q1].

In this approach, one requires an accurate estimate on the spectral gap of the generator in a finite volume. This estimate is obtained in [LY] under certain mixing assumptions, summarized as assumptions A.1-A.3 in [LY]. These assumptions are imposed on the canonical Gibbs states and are hard to check. They are replaced in [Y2] by some mixing conditions on the grand canonical Gibbs states, to be stated as assumption (A) in Section 2. Indeed, in that paper a much stronger inequality, the logarithmic Sobolev inequality, is obtained. The assumption A is the usual DobrushinShlosman mixing condition and holds, e.g., for ferromagnetic Ising models up to the critical point [MOS, N] in dimension $d=2$ and at sufficiently high temperatures for general lattice gas models.

Even with an accurate estimate on the spectral gap, the hydrodynamical limit for lattice gases at a finite temperature is by no means a straightforward extension of the earlier work. This is because one has to solve the equation (1.4) and the generator $\mathcal{L}$ exhibits the effect of interaction between particles, an effect that is not present in any of the earlier work on lattice gas models that deal exclusively with the infinite temperature case where the Gibbs measures are product measures. Besides the approach of [V1] and [Q], we also find that the formulation of in [EMY, LOY1], designed mainly for nonreversible nongradient systems, provides a convenient framework for this paper.

Two problems remain outstanding. One is establishing hydrodynamical limits of lattice gases without assuming mixing conditions; the other is hydrodynamical limit for nongradient models in continuum, namely, nongradient interacting Brownian motions. For the second problem, one has to derive accurate estimates of spectral gap for interacting Brownian motions in a finite volume. It seems that the approach of [LY, Y2] might be useful here. A more ambitious goal is to establish hydrodynamical limit without using any estimates on the spectral gap. We are not aware of any such approach that works for nongradient models.

This paper is organized as follows: In Section 2 we state the main result. An outline of proof is given in Section 3. The tightness is proved in Section 4, and an energy estimate is obtained in Section 5. The two-block estimate is proved in Section 6, and an eigenvalue estimate is in Section 7. In Section 8 and 9 we solve the fluctuationdissipation equation (1.4) in a precise sense. Finally, we prove some mixing conditions needed in this paper in Section 10.

2. Statement of the main result. Let $\Lambda=\Lambda_{L}$ be a cube of width $2 L+1$ in $Z^{d}$ and let $\eta=\left(\eta_{x}\right)_{x \in \Lambda_{L}}$ denote the configuration of a lattice gas where for each $x$, $\eta_{x} \in\{0,1\} . \eta_{x}=1$ if there is a particle at $x$ and $\eta_{x}=0$ means that the site $x$ is 
empty. For any two points in $Z^{d}$ we define two notions of distance:

$$
|x-y|_{1}:=\sum_{i=1}^{d}\left|x_{i}-y_{i}\right| ; \quad|x-y|:=\max _{i=1, \cdots d}\left|x_{i}-y_{i}\right|
$$

By the boundary $\partial \Lambda$ of $\Lambda$ we shall mean the complement $\Lambda^{c}$ of $\Lambda$ although in practice we need only be concerned with those sites in $\Lambda^{c}$ that are within a certain fixed distance (the range of the interaction) of $\Lambda$. A boundary condition $\omega$ is a configuration on $\partial \Lambda$ and $\eta \cup \omega$ is a configuration on $\Lambda \cup \partial \Lambda$. Let $F(\eta)$ be a local function depending on the configuration in some finite box of width $2 R+1$. Formally the Hamiltonian $H$ is given by

$$
H(\eta)=\sum_{x} F_{x}(\eta)
$$

Let us recall that $F_{x}(\eta)=F\left(\tau_{x} \eta\right)$ where $\tau_{x}$ is the translation $\left(\tau_{x} \eta\right)_{y}=\eta_{x+y}$. The energy $H_{\omega}$ of a configuration $\eta$ on $\Lambda$ with boundary condition $\omega$ is given by

$$
H_{\omega}(\eta)=\sum_{x \in \Lambda} F_{x}(\eta \cup \omega)
$$

We note that $H_{\omega}($.$) depends on \omega$ only through the configuration at sites in $\partial \Lambda$ that are with in a distance $2 R$ of $\Lambda$. We denote by $\mu_{\Lambda, \omega, \lambda}$ the finite volume Gibbs measure on $\Lambda$ with Hamiltonian $\mathrm{H}$, boundary condition $\omega$ and chemical potential $\lambda$, namely

$$
\mu_{\Lambda, \omega, \lambda}(\eta)=Z_{\Lambda, \omega, \lambda}^{-1} \exp \left[-H_{\omega}(\eta)+\lambda \sum_{x \in \Lambda} \eta_{x}\right]
$$

Here $Z_{\Lambda, \omega, \lambda}$ is the normalization constant (the partition function)

$$
Z_{\Lambda, \omega, \lambda}=\sum_{\eta} \exp \left[-H_{\omega}(\eta)+\lambda \sum_{x \in \Lambda} \eta_{x}\right]
$$

so that $\mu_{\Lambda, \omega, \lambda}$ is a probability measure on the space of configurations on $\Lambda$. For simplicity, we assume that the inverse temperature $\beta$ has been absorbed into $F$. When $\lambda=0$ we will drop the suffix $\lambda$.

Recall that the pressure $p(\lambda)$ of the lattice gas is defined by

$$
p(\lambda)=\lim _{\Lambda \rightarrow \infty}|\Lambda|^{-1} \log Z_{\Lambda, \omega, \lambda}
$$

Elementary theory of thermodynamics assures us that $p(\lambda)$ exists and is independent of the choice of boundary condition $\omega$. We also have the usual definition of the free energy $h(m)$, as the Legendre transform of $p$ :

$$
h(m)=\sup _{\lambda}[\lambda m-p(\lambda)]
$$

It is well known that the infinite volume Gibbs measures that are limits of the finite volume ones given by (2.4) exist, are characterized by the DLR equations, and in general may not be unique when there are phase transitions. Our resells are restricted to the single-phase region. Indeed, we need certain mixing conditions. 
Assumption A. let $\mu_{\Lambda, \omega, \lambda}$ denote a Gibbs measure on $\Lambda$ with boundary condition $\omega$ and chemical potential $\lambda$. Denote the corresponding density by $\rho=\rho(L, \lambda, \omega)$. Then there are constants $\gamma_{1}, \gamma_{2}$ and $\gamma_{3} \geq R+1$ such that for any two functions $f$ and $g$ with supports $S_{f}$ and $S_{g}$ we have

$$
\left|E_{\Lambda, \omega, \lambda}[f ; g]\right| \leq \gamma_{1} \rho(1-\rho) \exp \left[-\gamma_{2} \operatorname{dist}\left(S_{f}, S_{g}\right)\right]\|f\|_{\infty}\|g\|_{\infty}
$$

provided that the diameters of $S_{f}$ and $S_{g}$ are bounded by $\gamma_{3}$. Note that the constants are independent of the size of the cube $\Lambda$, the chemical potential and the boundary condition.

This exponential mixing condition is stronger than what we need in this paper and the exponential decay can be replaced by a weaker decay property. Since for most Gibbs measures, one usually obtains mixing conditions via the Dobrushin uniqueness condition or the cluster expansions that automatically yield exponential estimates, we will not attempt to find the optimal mixing condition.

We also need the concept of canonical Gibbs states. Let $\rho$ be a fixed positive number. Then the canonical Gibbs state with total density $\rho$ and boundary condition $\omega$ is the conditional probability of $\mu_{\Lambda_{L}, \omega}$ given that the total number of particles is equal to $L^{d} \rho$, namely, $\bar{\eta}:=L^{-d} \sum_{x \in \Lambda} \eta_{x}=\rho$. Certainly, $\rho$ has to be a rational number of some special form for the total number of particles to be an integer. One can avoid trouble by requiring that the total number of particles equal $\left[L^{d} \rho\right]$ rather than $L^{d} \rho$. Symbolically, we write the canonical measure as

$$
d \mu_{\Lambda, \omega, \rho}=\left.d \mu_{\Lambda, \omega}\right|_{\bar{\eta}=\rho}
$$

Note that since $\bar{\eta}$ is fixed we can replace $\mu_{\Lambda, \omega}$ by $\mu_{\Lambda, \omega, \lambda}$ with any $\lambda$. Define the canonical partition function

$$
Z_{\Lambda, \omega, \rho}^{c}=\sum_{\eta: \bar{\eta}=\rho} \exp \left[-H_{\Lambda, \omega}(\eta)\right]
$$

where $H_{\Lambda, \omega}=H_{\Lambda, \omega, 0}$. It should be emphasized that the mixing condition $\mathbf{A}$ by itself does not imply any mixing property for the canonical Gibbs measures. It is proved in [Y2] that the mixing condition $\mathbf{A}$ does imply some mixing property, though not as strong, for the canonical Gibbs measure. More importantly, exact estimate on the spectral gap as well as a Logarithmic Sobolev inequality are obtained under assumption A.

Denote by $b$ an unoriented bond $(x, y) \in \Lambda \times \Lambda$ with $|x-y|_{1}=1$. All bonds in this paper are unoriented unless otherwise specified. We have limited ourselves to nearest neighbor bonds mainly to simplify notation. As long as bonds with a fixed finite bound on length are used, the proofs will remain the same. Let $S_{b}$ be defined by

$$
\begin{aligned}
\left(S_{b} \eta\right)_{z}:=\left(\eta^{b}\right)_{z}=\left(\eta^{x, y}\right)_{z} & =\eta_{y} & & \text { if } z=x \\
& =\eta_{x} & & \text { if } z=y \\
& =\eta_{z} & & \text { otherwise }
\end{aligned}
$$

and define $T_{b}$ by

$$
T_{b} f(\eta)=f\left(\eta^{b}\right)-f(\eta)
$$


If $b$ happens to be the bond $(0, e)$ for some $e>0$ we will denote $T_{b}$ by $T_{e}$. Define $\mathcal{L}_{b}$ to be the symmetric generator defined by

$$
-\int f \mathcal{L}_{b} g d \mu=\frac{1}{2} \int c_{b}(\eta)\left[T_{b} f(\eta)\right]\left[T_{b} g(\eta)\right] d \mu
$$

and $\mathcal{L}=\sum_{b \in \Lambda} \mathcal{L}_{b}$. The rate $c_{b}(\eta)$ is assumed to be a local function that is translation invariant i.e. $c_{\tau_{x} b}\left(\tau_{x} \eta\right)=c_{b}(\eta)$ and bounded away from zero and infinity, i.e., $0<\delta<$ $c_{b}(\eta)<\delta^{-1}<\infty$ for some constant $\delta$. Explicitly, $\mathcal{L}_{b}$ is given by

$$
\mathcal{L}_{b} f=A(b, \eta) T_{b} f(\eta)
$$

where

$$
A(b, \eta)=(1 / 2)\left[\exp \left\{\left(T_{b} \log c_{b}\right)(\eta)-\left(T_{b} H\right)(\eta)\right\}+c_{b}(\eta)\right]
$$

When $b=(x, y)$ we sometimes use the notation $A(x, y, \eta)$ instead of $A(b, \eta)$. The operator $\mathcal{L}$ can serve as the infinitesimal generator of a Markov process $\eta(t)$ of configurations changing in time. We could consider either the infinite lattice $\mathbb{Z}^{d}$, a finite box $\Lambda$ with boundary conditions $\omega$ or a periodic lattice of size $L$. In all these cases the Markov Process will be reversible with respect to the corresponding Gibbs measure (in the infinite volume situation, with respect to any measure that satisfies the DLR equations ). We shall for the present be concerned mainly with the periodic case with $L \simeq \varepsilon^{-1}$ and we denote by $\mu_{\varepsilon}$ the corresponding Gibbs measure with $\lambda=0$. We will speed time up by a factor of $\varepsilon^{-2}$ so that the generator is $\varepsilon^{-2} \mathcal{L}$. Let us start the system initially with a distribution having density $f_{0}^{\varepsilon}$ with respect to $\mu_{\varepsilon}$. We denote by $P_{\varepsilon}^{f_{0}}$ the corresponding Markov Process with generator $\varepsilon^{-2} \mathcal{L}$ and initial distribution $f_{0}^{\varepsilon} d \mu_{\varepsilon}$ on the space of trajectories $\eta(\cdot)$ in a fixed macroscopic time interval $[0, T]$.

If $f_{t}^{\varepsilon}$ is the density of the system at time $t$ relative to the measure $\mu_{\varepsilon}$. Then $f_{t}^{\varepsilon}$ satisfies the forward equation

$$
\partial_{t} f_{t}^{\varepsilon}=\epsilon^{-2} \mathcal{L} f_{t}^{\varepsilon}
$$

We have, of course, already rescaled time with a diffusive rescaling $t \rightarrow \epsilon^{-2} t$ and by considering the evolution of empirical measure defined by

$$
\nu_{\epsilon}(\theta, t)=\varepsilon^{d} \sum_{z \in \Lambda} \delta(\theta-\epsilon z) \eta_{z}(t)
$$

where $\theta \in \mathbf{T}^{d}$, we are rescaling space by a factor of $\varepsilon$. Because $\eta \in\{0,1\}$, the relation (2.16) induces (from $P_{\varepsilon}$ ) a distribution, $Q_{\epsilon}^{f_{0}}$, of $\nu_{\epsilon}(\cdot, \cdot)$ on the Skorohod space $X=$ $D\left([0, T] \rightarrow M_{1}\left[\mathbf{T}^{d}\right]\right)$, where $M_{1}\left(\mathbf{T}^{d}\right)$ is the space of nonnegative measures on $\mathbf{T}^{d}$ with total mass bounded by 1 . The space $M_{1}\left(\mathbf{T}^{d}\right)$ is compact under the topology of weak convergence. We now describe the hydrodynamical limit. As $\epsilon \rightarrow 0$, one expects the distribution $Q_{\varepsilon}^{f_{0}}$ of empirical measures to converge to $\delta_{m(\theta, \cdot) d \theta}$, the Dirac measure concentrated on the trajectory $m(\theta, \cdot) d \theta$ where $m(t, \theta)$ satisfies the following nonlinear diffusion equation:

$$
\frac{\partial}{\partial t} m(\theta, t)=\nabla_{\theta}\left(D(m(\theta, t)) \nabla_{\theta} m(\theta, t)\right) ; \quad m(\theta, 0)=m_{0}(\theta)
$$

Here $m_{0}(\theta)$ is the initial density determined by the initial distribution $f_{0}^{\varepsilon} d \mu_{\varepsilon}$ and $D(m)$ is the diffusion (matrix) coefficient given by the Green-Kubo formula to be 
described later. Clearly, in order for this to make sense, we need at least that the initial distribution $f_{0}^{\varepsilon} d \mu_{\varepsilon}$ satisfies

$$
\limsup _{\varepsilon \rightarrow 0} P^{f_{0}^{\varepsilon}}\left[\left|\int \nu_{\varepsilon}(\theta, 0) J(\theta) d \theta-\int m_{0}(\theta) J(\theta) d \theta\right|>\delta\right]=0
$$

for any smooth test function $J(\cdot)$ on $\mathbf{T}^{d}$ and $\delta>0$. We shall make this assumption for the rest of this paper. Since it is the only condition we need on the initial distribution, we may as well have deterministic initial conditions and suppose that at 0 we start from a nonrandom configuration $\xi^{\varepsilon}$. We shall denote by $P_{\varepsilon}^{\xi}$ the distribution of the process with this initial condition and the corresponding distribution on $X$ by $Q_{\varepsilon}^{\xi}$. When no confusion arises, we shall drop the label $\varepsilon$. The condition (2.18) takes the form

$$
\limsup _{\varepsilon \rightarrow 0}\left|\frac{1}{L^{d}} \sum_{z} J\left(\frac{z}{L}\right) \xi_{z}-\int m_{0}(\theta) J(\theta) d \theta\right|=0
$$

The Green-Kubo formula for the diffusion coefficient can be written in several ways $[\mathrm{S}]$. Let $\tau_{x}$ denote the translation by $x$, namely,

$$
\left(\tau_{x} \eta\right)(y)=\eta(y-x), \quad \text { and } \tau_{x} g(\eta)=g\left(\tau_{x} \eta\right)
$$

Denote by $\mu_{m}$ the infinite volume Gibbs state with density $m$ and $\chi(m)$ its compressibility defined by

$$
\chi(m)=\sum_{x \in \mathbb{Z}^{d}} E^{\mu_{m}}\left[\eta_{0} ; \eta_{x}\right]
$$

here the truncated correlation is defined by

$$
E^{\mu_{m}}[f ; g]=E^{\mu_{m}}[f g]-E^{\mu_{m}}[f] E^{\mu_{m}}[g]
$$

The following characterization is due to [V1]. For all vector $\alpha$ we have

$$
<\alpha, D(m) \alpha>=\frac{1}{2 \chi(m)} \inf _{g} E^{\mu_{m}}\left[\sum_{|e|=1, e>0} c_{e}(\eta)\left(\alpha_{e} \nabla_{e} \eta_{0}-T_{e} \sum_{x \in \mathbb{Z}^{d}} \tau_{x} g\right)^{2}\right]
$$

where the infimum is over all local functions $g, T$ is defined in (2.11) and $\nabla_{e}$ is defined by

$$
\left(\nabla_{e}\right) h_{x}=h_{x+e}-h_{x}
$$

for any function on $\mathbb{Z}^{d}$. Since $g$ is local, $T_{e} \sum_{x \in \mathbb{Z}^{d}} \tau_{x} g$ makes sense even though the infinite sum $\sum_{x \in \mathbb{Z}^{d}} \tau_{x} g$ does not. Since the right hand side of (2.22) is a quadratic expression in the $\alpha$ and $g$, and the infimum is taken over $g$ belonging to the linear subspace of all local functions, the left hand side is quadratic in $\alpha$ and defines a symmetric nonnegative definite matrix $D(m)$ for each $m$. Moreover because (2.22) defines the diffusion coefficient as an infimum we get upper bounds for $D(m)$. It is much harder to obtain a lower bound for the diffusion coefficient. Such a bound is obtained in [SY] which states that in matrix sense

$$
D(m) \geq C[\chi(m)]^{-1} I
$$


for some constant depending on the Hamiltonian. We remark that this bound is independent of phase transition and does not require any mixing conditions. However, we do need the mixing conditions to establish rigorously that the hydrodynamic limit can be taken to get a nonlinear diffusion equation with coefficients given by (2.22).

The uniqueness of (2.17) is quite subtle. If the diffusion coefficient is a scalar, then the uniqueness follows easily. Another situation where uniqueness holds is when the diffusion coefficient is Lipshitz continuous in $m$ and satisfies uniform ellipticity bounds. Under this assumption, the general theory guarantees the existence of Lipshitz continuous solutions. Suppose $v$ is such a solution and $u$ is a weak solution satisfying an energy estimate. Then we have

$$
\begin{aligned}
\partial_{t} \int(u-v)^{2}(\theta) d \theta= & -\int \nabla(u-v)(\theta)[D(u) \nabla u-D(v) \nabla v](\theta) d \theta \\
= & -\int \nabla(u-v)(\theta) D(u(\theta))[\nabla u-\nabla v](\theta) d \theta \\
& +\int \nabla(u-v)(\theta)[D(u)-D(v)](\theta) \nabla v(\theta) d \theta
\end{aligned}
$$

From the Schwarz inequality the last term is bounded by

$$
\begin{aligned}
& \int \nabla(u-v)[D(u)-D(v)] \nabla v d \theta \\
& \leq \gamma \int[\nabla(u-v)]^{2} d \theta+\gamma^{-1} \int[D(u)-D(v)]^{2}(\nabla v)^{2} d \theta
\end{aligned}
$$

Since $v$ is Lipshitz continuous, the sup-norm of $\nabla v$ is bounded. Together with the Lipshitz continuity of $D$, the last term is bounded by

$$
C \gamma^{-1} \int[D(u)-D(v)]^{2} d \theta \leq C \gamma^{-1} \int(u-v)^{2} d \theta
$$

By choosing $\gamma$ small enough, we have thus proved that

$$
\partial_{t} \int(u-v)^{2} d \theta \leq C \gamma^{-1} \int(u-v)^{2} d \theta
$$

This proves the uniqueness. Our main result of this paper is the following theorem:

Theorem 2.1 Suppose that the Gibbs measure satisfies the mixing condition $\mathbf{A}$. Suppose the initial data $\xi^{\varepsilon}$ satisfies the condition (2.18) and that (2.17) with the initial condition specified by (2.18) has a unique solution $m(\theta, t)$ in the class of weak solutions satisfying the energy estimate. Then

$$
\limsup _{\varepsilon \rightarrow 0} P_{\varepsilon}^{\xi}\left[\left|\varepsilon^{d} \sum_{x \in \Lambda} J(\varepsilon x) \eta_{x}(t)-\int J(\theta) m(\theta, t) d \theta\right|>\delta\right]=0
$$

Here $P_{\varepsilon}^{\xi}$ refers to the process with initial data $\xi^{\varepsilon}$.

Note that one can start from a deterministic initial data as long as (2.18) holds. The Lipshitz continuity of the diffusion coefficient has not been proved, though it is proved for the self-diffusion coefficient in [V3] and for asymmetric simple exclusion in 
[LOY2]. In the special case $d=1$ or when the Hamiltonian is isotropic (Lemma 8.3), the diffusion coefficient is a scalar and Theorem 2.1 is then always valid.

As a technical remark, all error terms arising in the proof of Theorem 2.1 will be shown to be superexponentially small. Hence our estimates already give an upper bound to the large deviation probability in the hydrodynamical limit. One needs a corresponding lower bound to complete the large deviation theory. Since this has been carried out carefully in [DV, Q2] for Ginzburg-Landau models, in [QRV] for the symmetric simple exclusion processes with several colors and in [QY2] for lattice gas models for the incompressible Navier-Stokes equation, we will not pursue this direction here.

3. Outline of the proof. Our basic approach for proving the hydrodynamical limit consists of three steps: establishing the tightness of the measures $\left\{Q_{\varepsilon}^{\xi}: \varepsilon>0\right\}$, deriving an energy estimate that provides some regularity for functions in the support of any weak limit $Q$ of $Q_{\varepsilon}^{\xi}$ as $\varepsilon \rightarrow 0$ and identification of the support of $Q$ as weak solutions of (2.17). To prove tightness, from Prohorov's theorem, it suffices to prove the following estimate:

Lemma 3.1 (Tightness) For any initial data $\xi^{\varepsilon}$, any smooth test function $J$ and any $\delta>0$ we have

$\limsup _{\alpha \rightarrow 0} \limsup _{\varepsilon \rightarrow 0} P_{\varepsilon}^{\xi}\left[\sup _{|s-t| \leq \alpha, 0 \leq s, t \leq T}\left|\varepsilon^{d} \sum_{x} J(\varepsilon x) \eta_{x}(t)-\varepsilon^{d} \sum_{x} J(\varepsilon x) \eta_{x}(s)\right| \geq \delta\right]=0$

Note that

$$
\limsup _{\varepsilon \rightarrow 0}\left|\varepsilon^{d} \sum_{x} J(\varepsilon x) \eta_{x}\right| \leq\|J\|_{1}
$$

Hence any limit point $Q$ of $Q_{\varepsilon}^{\xi}$ is supported on measure valued functions on [0,T], that are absolutely continuous w.r.t. the Lebegue measure, with densities $m(\theta, t)$ bounded between 0 and 1 . At this point we only know that $m(\theta, t)$ is a measurable function. The next step is to prove that it satisfies a basic energy estimate.

Lemma 3.2 (Energy Estimate) Suppose $Q$ is any limit point for $Q_{\varepsilon}^{\xi}$. There is a constant $C$ such that

$$
E^{Q}\left\{\int_{0}^{T} d t \int_{\mathbf{T}^{d}} d \theta\left(\nabla_{\theta} m(\theta, t)\right)^{2}\right\} \leq C
$$

Finally, we have to prove that $Q$ is supported on densities $m$ that satisfy the equation (2.17) in the weak sense. Denote by $\bar{\eta}_{x, \ell}$ the average density of $\eta$ in a cube of width $2 \ell+1$, namely,

$$
\bar{\eta}_{x, \ell}=(2 \ell+1)^{-d} \sum_{y:|y-x| \leq \ell} \eta_{y}:=A v_{y:|y-x| \leq \ell} \eta_{y}
$$

This operation of averaging over a cube of side $2 \ell+1$ centered at $x$ will be denoted by $A v_{y:|y-x| \leq \ell}$ and can be performed on other random fields defined on the lattice. 
Theorem 3.3 (Identification of the Equation) For any $\delta>0$, let

$$
\begin{aligned}
& B_{a, b, \delta}^{J} r=\left\{\eta(\cdot): \sup _{0 \leq t \leq T} \mid \varepsilon^{d} \sum_{x} J(\varepsilon x) \eta_{x}(t)-\varepsilon^{d} \sum_{x} J(\varepsilon x) \eta_{x}(0)\right. \\
& \left.+\int_{0}^{t}(2 b)^{-1} \varepsilon^{d} \sum_{x, e, e^{\prime}}\left[\eta_{x+b \varepsilon^{-1} e}(s)-\eta_{x-b \varepsilon^{-1} e}(s)\right] D_{e, e^{\prime}}\left(\bar{\eta}_{x, a \varepsilon^{-1}}(s)\right)\left(\nabla_{e^{\prime}} J\right)(\varepsilon x) d s \mid>\delta\right\}
\end{aligned}
$$

denote the event that leads to the violation of the limiting equation for the smooth test function $J$; here $D$ is the diffusion coefficient given by (2.22). Then

$$
\limsup _{a \rightarrow 0} \limsup _{b \rightarrow 0} \limsup _{\epsilon \rightarrow 0} P_{\varepsilon}^{\xi}\left[B_{a, \delta}^{J}\right]=0
$$

Clearly, Theorem 2.1 follows from these three results and the uniqueness of the weak solution for the equation (2.17) satisfying the energy estimate.

The tightness will be proved in next section. The energy estimate will be proved in section 5. We now outline the proof of Theorem 3.3.

Proof of Theorem 3.3.

Step 1: Recall that the current is defined by the equation

$$
\mathcal{L} \eta_{x}=-\sum_{e>0} \nabla_{e}^{-} w_{x, x+e}(\eta)
$$

where $\sum_{e>0}$ denotes the summation over the unit vectors in the positive coordinate directions and for any (possibly random) function $h(x, \cdot)=h_{x}(\cdot)$ on the lattice

$$
\nabla_{e}^{-} h(x, \cdot)=h(x, \cdot)-h(x-e, \cdot) .
$$

We shall follow the convention of using $e$ to denote a unit vector in the positive coordinate direction. Explicitly, the current is given by

$$
w_{x, x+e}(\eta)=A(x, x+e, \eta)\left(\eta_{x}-\eta_{x+e}\right)
$$

where $A$ is the jump rate defined in (2.14). We have the summation by parts identity

$$
\sum_{x} g(x) \nabla_{e}^{-} h(x)=-\sum_{x}\left(\nabla_{e} g(x)\right) h(x)
$$

where $\nabla$ is defined in (2.23) and $\sum_{x}$ denotes the summation over the lattice sites on the periodic lattice with width $\varepsilon^{-1}$. This convention will be followed in the rest of this paper unless otherwise stated. From stochastic calculus,

$$
\begin{array}{r}
\varepsilon^{d} \sum_{x} J(\varepsilon x) \eta_{x}(t)-\varepsilon^{d} \sum_{x} J(\varepsilon x) \eta_{x}(0)=\int_{0}^{t} U(\eta(s)) d s+M_{N}(t), \\
U(\eta)=\varepsilon^{d-1} \sum_{x} \sum_{e>0}\left(\varepsilon^{-1} \nabla_{e} J(\varepsilon x)\right) w_{x, x+e}(\eta) \\
M_{N}(t)=\int_{0}^{t} \varepsilon^{d} \sum_{x} \sum_{e>0} \nabla_{e} J(\varepsilon x) \eta_{x}(s) d M_{x, x+e}(s)
\end{array}
$$


where $M_{x, x+e}(t)$ is a martingale with quadratic variation

$$
d<M_{x, x+e}(t), M_{x, x+e}(t)>=\varepsilon^{-2} A(x, x+e, \eta) d t .
$$

Clearly,

$$
d<M_{N}(t), M_{N}(t)>=\varepsilon^{2 d} \sum_{x}\left(\varepsilon^{-1} \nabla_{e}^{-} J(\varepsilon x)\right)^{2} \eta_{x}(t) A(x, x+e, \eta) d t \leq C(J) \varepsilon^{d} d t
$$

Hence we can neglect the martingale term.

Step 2 (Fluctuation-dissipation equation in equilibrium) It remains to identify the current as $-D\left(\eta_{x, a \varepsilon^{-1}}\right) \nabla \eta_{x}(s)$. This step is very subtle and is the key step in nongradient system. In fact, we can not expect the substitution $w_{x, x+e} \rightarrow-\sum_{e^{\prime}} D_{e, e^{\prime}}\left(\eta_{x, a \varepsilon^{-1}}\right)$ $\nabla_{e^{\prime}} \eta_{x}$ to hold as it stands. The correct statement is that the relation

$$
w_{x, x+e}=-\sum_{e^{\prime}} D_{e, e^{\prime}}\left(\eta_{x, a \varepsilon^{-1}}\right) \nabla_{e^{\prime}} \eta_{x}+\mathcal{L} g_{e}
$$

holds for some suitable choice of local functions $\left\{g_{e}\right\}$. For processes in equilibrium, a precise statement is given by the following Theorem 3.4. We will first need some notation.

Following [LOY1], we define the space

$$
\mathcal{G}=\left\{h: h \text { is a local function and } h \text { satisfies } E^{\mu}\left[h \mid \mathcal{F}_{s}\right]=0 \text { for some } s\right\},
$$

where $\mathcal{F}_{s}=\mathcal{F}_{0, s}$ and

$$
\mathcal{F}_{x, s}=\text { the } \sigma \text {-algebra generated by }\left\{\bar{\eta}_{s}\right\} \cup\left\{\eta_{y}:|y-x|>s\right\} .
$$

For latter usage, we also define the $\sigma$-algebra

$$
\mathcal{K}_{x}^{s}=\sigma \text {-algebra generated by }\left\{\eta_{y}|| x-y \mid \leq s\right\} .
$$

For any local function $h \in \mathcal{G}$ we define the variance

$$
V_{\ell}(h, y, \omega)=\ell^{d}\left\langle A v_{|x| \leq \ell_{1}} h_{x},\left(-\mathcal{L}_{\ell}\right)^{-1} A v_{|x| \leq \ell_{1}} h_{x}\right\rangle_{\mu_{\ell, y, \omega}}
$$

where $h_{x}=\tau_{x} h, \ell_{1}=\ell-\sqrt{\ell}$ and $\mu_{\ell, y, \omega}$ is the canonical Gibbs measure on $\Lambda_{\ell}$ with the boundary condition $\omega$ and total density $y$. From the choice of $\ell_{1}$ it follows that for any fixed $h \in \mathcal{G}$, for sufficiently large $\ell, \tau_{x} h$ depends only on the configuration inside the cube $\Lambda_{\ell}$ provided $x \in \Lambda_{l_{1}}$. Since the boundary condition $\omega$ on $\Lambda_{\ell}^{c}$ and total density $y$ in $\Lambda$ are specified by the $\sigma$-algebra $\mathcal{F}_{\ell}$, the quantity $V_{\ell}(h, y, \omega)$, is an $\mathcal{F}_{\ell}$ measurable function $V_{\ell}(h, \eta)$ of the configuration $\eta$ on $\mathbb{Z}^{d}$. We define

$$
V(h, \beta)=\limsup _{\substack{\beta^{\prime} \rightarrow \beta \\ \ell \rightarrow \infty}} E^{\mu_{\beta^{\prime}}}\left[V_{\ell}(h, \eta)\right] .
$$

Here $\mu_{\beta}$ is the (unique) infinite volume Gibbs state with density $\beta$. For a local function $h \notin \mathcal{G}$, i.e. not satisfying the mean 0 condition $E^{\mu}\left[h \mid \mathcal{F}_{s}\right]=0$, we define $q_{x, k}(\omega)=$ 
$E^{\mu_{\ell, y, \omega}}\left[h_{x} \mid \mathcal{F}_{x, k}\right]$ where $\mathcal{F}_{x, k}$ defined in (3.8) is the $\sigma$-algebra generated by $\bar{\eta}_{x, k}$ and $\left\{\eta_{y}:|y-x|>k\right\}$. Then we define

$$
V(h, \beta)=\limsup _{\substack{k \rightarrow \infty \\ \beta^{\prime} \rightarrow \beta}} V\left(h-q_{k}, \beta^{\prime}\right)
$$

We now state the Theorem.

THEOREM 3.4 Fix a density $\beta$. Suppose $\mathbf{g}=\left\{g_{e}(\eta)\right\}$ is a local function (with $d$ components) of $\eta$. Let

$$
\phi_{e}(\mathbf{g})=w_{0, e}+\mathcal{L} g_{e}(\eta)+\sum_{e^{\prime}} D_{e, e^{\prime}}(\beta) \nabla_{e^{\prime}} \eta_{0}
$$

where the diffusion $D(\beta)$ is given by (2.22). Then for any $\left\{\alpha_{e}\right\}$,

$$
\inf _{\mathbf{g}} V\left(\sum_{e} \alpha_{e} \phi_{e}(\mathbf{g}), \beta\right)=0 .
$$

Assuming this result, we continue to prove Theorem 3.3. The minimizing sequence for (3.14) depends on the density $\beta$. Since, from the definition, the variance $V$ is upper semi-continuous, we have the following Corollary:

Corollary 3.5 For any $\left\{\alpha_{e}\right\}$ and $\delta>0$ there exists $\mathbf{g}=\left\{g_{e}(\beta, \eta)\right\}$, which is smooth as a function of $\beta$ and is local as a function of $\eta$, such that

$$
\sup _{\beta} V\left(\sum_{e} \alpha_{e} \phi_{e}(\beta, \mathbf{g}), \beta\right) \leq \delta ;
$$

where

$$
\phi_{e}(\beta, \mathbf{g})=w_{0, e}+\mathcal{L} g_{e}(\beta, \eta)+\sum_{e^{\prime}} D_{e, e^{\prime}}(\beta) \nabla_{e^{\prime}} \eta_{0}
$$

Step 3 (Insertion of local functions) To use theorem 3.5, we have to extend it to nonequilibrium process and insert the local function $\mathbf{g}$ into the stochastic equation (3.5). We first replace $J$ by $J * \omega_{\ell_{1}}$ where $\omega_{\ell_{1}}$ is the normalized characteristic function for a cube of size $2 \ell_{1}+1$ with $\ell_{1}=\ell-\sqrt{\ell}$, namely,

$$
\omega_{\ell_{1}}(y)= \begin{cases}\left(2 \ell_{1}+1\right)^{-d} & \text { if }|y| \leq \ell_{1} \\ 0 & \text { otherwise }\end{cases}
$$

Let $\boldsymbol{\Phi}_{x}^{\mathbf{g}}$ be defined as the vector with components

$$
\Phi_{x, e}^{\mathbf{g}}=A v_{|y-x| \leq \ell_{1}}\left[w_{y, y+e}+\mathcal{L} g_{e}\left(\bar{\eta}_{x, \ell}, \tau_{y} \eta\right)\right]
$$

Note that $\Phi$ depends additionally on $\ell$ which does not appear explicitly in the indices. Then

$$
\begin{aligned}
\varepsilon^{d} \sum_{x}\left(J(\varepsilon x) * \omega_{\ell_{1}}\right) \eta_{x}(t)-\varepsilon^{d} \sum_{x}\left(J(\varepsilon x) * \omega_{\ell_{1}}\right) \eta_{x}(0) & +\Omega_{\mathbf{g}}(\eta(t))-\Omega_{\mathbf{g}}(\eta(0)) \\
& =\int_{0}^{t} U(\eta(s), \mathbf{g}) d s+M_{\mathbf{g}}(t)
\end{aligned}
$$


where

$$
\begin{aligned}
\Omega_{\mathbf{g}}(\eta) & =\varepsilon^{d+1} \sum_{x, e>0}\left[\left(\varepsilon^{-1} \nabla_{e} J(\varepsilon x)\right) * \omega_{\ell_{1}}\right] g_{e}\left(\bar{\eta}_{x, \ell}, \tau_{y} \eta\right) \\
U(\eta, \mathbf{g}) & =\varepsilon^{d-1} \sum_{x, e>0}\left(\varepsilon^{-1} \nabla_{e} J(\varepsilon x)\right) \Phi_{x, e}^{\mathbf{g}}
\end{aligned}
$$

The explicit form of $M_{\mathbf{g}}(t)$, the martingale term, is not important. One can check easily that

$$
d<M_{\mathbf{g}}(t), M_{\mathbf{g}}(t)>\leq C(J, \mathbf{g}) \varepsilon^{d} d t
$$

and thus it is negligible for proving Theorem 3.3. Also, for any local function $g$

$$
\sup _{\eta}\left|\Omega_{\mathbf{g}}(\eta)\right| \leq C \varepsilon
$$

and is negligible too.

The generator $\mathcal{L}$ in (3.17) acts on the variables $\eta$ as well as $\bar{\eta}_{x, \ell}$ when applied to the function $g_{e}$. We first prove that the contribution of $\mathcal{L}$ acting on $\bar{\eta}_{x, \ell}$ is negligible. For this purpose, we need the next lemma relating the nonequilibrium process to equilibrium one.

LEMMA 3.6 Recall that $P_{\varepsilon}^{\xi}$ denotes the process starting from $\xi$ and $P_{\varepsilon}:=P^{\mu_{\varepsilon}}$ is the equilibrium process starting from $\mu_{\varepsilon}$ defined in section 2 after (2.16). Then there is a constant $\kappa$ depending only on the Hamiltonian such that

$$
\log \left\|\frac{d P_{\varepsilon}^{\xi}}{d P_{\varepsilon}}\right\|_{p} \leq \kappa \varepsilon^{-d}, \quad 1 \leq p \leq \infty .
$$

As a consequence,

$$
P_{\varepsilon}^{\xi}(A) \leq \exp \left[\kappa \varepsilon^{-d}\right] P_{\varepsilon}(A)
$$

In particular, for any events $A_{\varepsilon}$ which are superexponentially small in equilibrium, i.e.,

$$
\limsup _{\varepsilon \rightarrow 0} \varepsilon^{d} \log P_{\varepsilon}(A)=-\infty
$$

we have

$$
\limsup _{\varepsilon \rightarrow 0} \varepsilon^{d} \log P_{\varepsilon}^{\xi}(A)=-\infty
$$

The proof of this Lemma is trivial. One simply notes that, since the dynamics of the two processes are the same, $d P_{\varepsilon}^{\xi} / d P_{\varepsilon}=d \mu_{\delta(\xi)} / d \mu_{\varepsilon}$; here $\mu_{\delta(\xi)}$ is the delta measure of the configuration $\xi$. From the explicit expression of $d \mu_{\varepsilon}$, we can get a lower bound on $\mu_{\varepsilon}(\xi)$ of the form $\log \mu_{\varepsilon}(\xi) \geq-\kappa \varepsilon^{-d}$ and (3.20) follows and we have proved this Lemma.

This lemma shows that the notion of superexponentially small events is the same under equilibrium and nonequilibrium processes. The following lemma is a simple consequence of the Feynman-Kac formula and allows us to transform equilibrium exponential estimates into eigenvalue problems.

LEMMA 3.7 Let $L$ be the generator of a Markov process $x(t)$ with a reversible invariant measure $\nu$. Let

$$
u(x, t)=E_{x}\left[\exp \left\{\int_{0}^{T} V(x(s)) d s\right\}\right]
$$


where $E_{x}[\cdot]$ denotes the expectation with respect to the process starting at $x$. Then

$$
\begin{aligned}
\frac{1}{T} \log \int u(x, T) d \nu(x) & \leq \sup \operatorname{spec}\{V-(-\mathcal{L})\} \\
& =\sup _{\int f d \nu=1, f \geq 0}\left\{\int V f d \nu-\mathcal{D}(\sqrt{f})\right\}
\end{aligned}
$$

Here $\mathcal{D}(f)=-\int f L f d \nu$ is the Dirichlet form.

Proof. By the Feynman-Kac formula, $u$ solves the equation $\frac{\partial u}{\partial t}=L u+V u$ with the initial condition $u(0, x) \equiv 1$. Multiplying the equation by $u$ and integrating by parts we obtain

$$
\frac{\partial}{\partial t} \frac{1}{2} \int u^{2} d \nu=\int V u^{2} d \nu-\mathcal{D}(u) \leq \sup \operatorname{spec}[V-(-\mathcal{L})] \int u^{2} d \nu
$$

Therefore

$$
\frac{1}{T} \log \int u d \nu \leq \frac{1}{2 T} \log \int u^{2} d \nu \leq \sup \operatorname{spec}[V-(-\mathcal{L})]
$$

Applying these two lemmas to our setting, we have

LEMMA 3.8 The event $A=\left\{\left|\int_{0}^{T} V(\eta(s)) d s\right|>\delta\right\}$ is super exponentially small if and only if for all $\gamma>0$

$$
\limsup _{\varepsilon \rightarrow 0} \sup \operatorname{spec}\left\{V-\gamma^{-1} \varepsilon^{-2+d}(-\mathcal{L})\right\} \leq 0
$$

Proof. Suppose the process is in equilibrium. Then the corollary follows from Lemma 3.7 and Chebyshev's inequality. For a general nonequilibrium process, we only have to use Lemma 3.6 to prove the Lemma. Note that we do not have to specify the initial distribution of the process.

We now return to the local function $\mathrm{g}$. Let

$$
\mathcal{L}_{y, s}=\sum_{b \in \Lambda_{y, s}} \mathcal{L}_{b}
$$

where $b \in \Lambda_{y, s}$ means that both ends of the bond $b$ lie in the box $\Lambda_{y, s}$ of width $2 s+1$ centered at $y$. We have

$$
\mathcal{L} g_{e}\left(\bar{\eta}_{x, \ell}, \tau_{y} \eta\right)=\mathcal{L}_{y, \sqrt{\ell}} g_{e}\left(\bar{\eta}_{x, \ell}, \tau_{y} \eta\right)+\sum_{\substack{b \in \partial \Lambda_{x}, \ell \\ b \notin \Lambda_{y, \sqrt{\ell}}}} \mathcal{L}_{b} g_{e}\left(\bar{\eta}_{x, \ell}, \tau_{y} \eta\right)
$$

provided $\sqrt{\ell}$ is bigger than the range of the local function $g_{e}$, which we shall assume. By $b \in \partial \Lambda$, we mean that the bond straddles $\Lambda$ and $\Lambda^{c}$. By the definition of $\mathcal{L}$ and Schwarz's inequality, we have for any function $g$,

$$
\begin{aligned}
\varepsilon^{-1} \int f \mathcal{L}_{b} g d \mu_{\varepsilon} & =\varepsilon^{-1} \int c_{b}(\eta)\left[f\left(\eta^{b}\right)-f(\eta)\right] T_{b} g d \mu_{\varepsilon} \\
& \leq \varepsilon^{-2} \ell^{-d} \int c_{b}(\eta)\left[\sqrt{f}\left(\eta^{b}\right)-\sqrt{f}(\eta)\right]^{2} d \mu_{\varepsilon} \\
& +\ell^{d} \int c_{b}(\eta)\left[\sqrt{f}\left(\eta^{b}\right)+\sqrt{f}(\eta)\right]^{2}\left(T_{b} g\right)^{2} d \mu_{\varepsilon}
\end{aligned}
$$


Since $g_{e}$ is smooth as a function of its first variable, we have $T_{b} g_{e}\left(\bar{\eta}_{x, \ell}, \tau_{y} \eta\right) \leq C \ell^{-d}$ for any boundary bond $b \in \partial \Lambda_{x, \ell}$. Note that $T_{b}$ does not act on $\tau_{y} \eta$ since $g_{e}$ is a local function and $\ell$ is large enough. Since the number of boundary terms is of order $\ell^{d-1}$, we have for any smooth function $J$ and any $\gamma>0$,

$\underset{\ell, \varepsilon}{\limsup \sup s p e c}\left[\epsilon^{-1} \sum_{x}\left(J(\varepsilon x) \sum_{\substack{b \in \Lambda_{x}, \ell \\ b \notin \Lambda_{y, \sqrt{\ell}}}} \mathcal{L}_{b} g\left(\bar{\eta}_{x, \ell}, \tau_{y} \eta\right)\right)-\gamma^{-1} \epsilon^{-2} A v_{b}\left(-\mathcal{L}_{b}\right)\right] \leq 0$

where

$$
\limsup _{\ell, \varepsilon}=\limsup _{\ell \rightarrow \infty} \limsup _{\epsilon \rightarrow 0}
$$

We will use this notation repeatedly when we want to take successive limsups in a specified order. Hence we can replace $\mathcal{L}$ in $(3.17)$ by $\mathcal{L}_{y, \sqrt{\ell_{1}}}$, namely, we can assume that $\mathcal{L}$ in (3.17) does not act on $\bar{\eta}_{x, \ell}$. Throughout the rest of this paper, while computing $\mathcal{L} g_{e}\left(\bar{\eta}_{x, \ell}, \tau_{y} \eta\right)$, we will therefore be justified in keeping only the first term on the right side of (3.26), and effectively ignoring the action of the generator on the first variable of $g_{e}$.

Step 4 (Eigenvalue estimate) Our goal is to prove that (1.4) holds in nonequilibrium. The basic tool is Lemma 3.8. In order to apply this lemma, we will need to estimate some eigenvalues, to be proved in section 7 .

Theorem 3.9 (Eigenvalue Estimate) Suppose $\mathbf{J}=\left\{J_{e}\right\}$ is a smooth vector valued function and $\mathbf{g}=\left\{g_{e}(\beta, \eta)\right\}$ is smooth as a function of $\beta$ and is a local function of $\eta$. Recall the definitions of $\Phi \mathrm{g}$ (3.17) and $\phi_{e}$ (3.13). Let us define the density gradients $\Psi_{\varepsilon, c}(x)$ componentwise by $\Psi_{\varepsilon, c, e}(x)=\varepsilon(2 c)^{-1}\left[\eta_{y+c \varepsilon^{-1} e}-\eta_{y-c \varepsilon^{-1} e}\right]$. Then for any $\gamma>0$, there exists a constant $C(\gamma)$ such that

$$
\begin{gathered}
\limsup _{\ell, a, c, \varepsilon} \sup \operatorname{spec}\left[\epsilon^{-1}\left(\mathbf{J}(\varepsilon x), \Phi_{x}^{\mathbf{g}}+D\left(\bar{\eta}_{x, a \varepsilon^{-1}}\right) A v_{|y-x| \leq \ell_{1}}\left[\Psi_{\varepsilon, c}(y)\right]-\gamma \epsilon^{-2} A v_{b}\left(-\mathcal{L}_{b}\right)\right]\right. \\
\leq C(\gamma)\|J\|_{\infty}^{2} \sup _{\sum_{e} \alpha_{e}^{2}=1} \sup _{\beta} V\left(\sum_{e} \alpha_{e} \phi_{e}(\beta, \mathbf{g}), \beta\right) ;
\end{gathered}
$$

here $(\mathbf{J}, \tilde{\mathbf{J}})=A v_{x} \sum_{e>0,|e|=1} J_{e}(\varepsilon x) \tilde{J}_{e}(\varepsilon x)$ and the meaning of the limit is given in (3.27).

We can now conclude Theorem 3.3. From (3.14) we can choose $g$ so that the variance on the right side of (3.28) is arbitrarily small for any fixed $\gamma$. Hence the infimum over $g$ of the left hand side of (3.28) is bounded above by zero. From Lemma 3.8 we can prove Theorem 3.3.

4. Tightness. In this section, we shall prove the tightness Lemma 3.1. Corresponding results are obtained in [GPV] and [V1] via the Garsia, Rodemich and Rumsey Lemma. We will however provide a more direct martingale argument that establishes tightness.

Recall the definition of current in (3.4). We have the exponential martingale: $e^{Z(\beta, t)}$ where

$$
Z(\beta, t)=\beta \sum_{x} J(\varepsilon x) \eta_{x}(t)-\beta \sum_{x} J(\varepsilon x) \eta_{x}(0)-\int_{0}^{t}(\beta U(\eta(s))+\Omega(\eta(s))) d s
$$




$$
U(\eta)=\varepsilon^{-1} \sum_{x}\left(\varepsilon^{-1} \nabla_{e}^{-} J(\varepsilon x)\right) w_{x, x+e}(\eta)
$$

and

$$
\Omega(\eta)=\exp \left(-\beta \sum_{x} J(\varepsilon x) \eta_{x}\right) \mathcal{L} \exp \left(\beta \sum_{x} J(\varepsilon x) \eta_{x}\right)-\beta U(\eta)
$$

Since $J$ is smooth,

$$
\sup _{\eta}|\Omega(\eta)| \leq C(J) \beta^{2} \varepsilon^{-d}, \quad \sup _{\eta}|U(\eta)| \leq C(J) \beta^{2} \varepsilon^{-1-d} .
$$

LEMMA 4.1 For each smooth $J$, for sufficiently small $\varepsilon$ we have

$$
\begin{aligned}
& P_{\varepsilon}^{\xi}\left[\sup _{0 \leq j \leq T \varepsilon^{-2}} \sup _{j \varepsilon^{2} \leq t \leq(j+1) \varepsilon^{2}}\left|\varepsilon^{d} \sum_{x} J(\varepsilon x) \eta_{x}(t)-\varepsilon^{d} \sum_{x} J(\varepsilon x) \eta_{x}\left(j \varepsilon^{2}\right)\right| \geq \delta\right] \\
& \quad \leq \exp \left(-\delta \varepsilon^{-d / 8)}\right.
\end{aligned}
$$

Proof. Let $\beta=1$. From the martingale inequality we have

$$
P_{\varepsilon}^{\xi}\left[\sup _{0 \leq s \leq t} Z(1, s) \geq \varepsilon^{-d} \delta\right] \leq \exp \left(-\delta \varepsilon^{-d}\right) E_{\varepsilon}^{\xi}\left[e^{Z(1, t)}\right]=\exp \left(-\delta \varepsilon^{-d}\right)
$$

From (4.2), we have, assuming without loss of generality that $\varepsilon \leq 1$,

$$
\begin{aligned}
& P_{\varepsilon}^{\xi}\left[\sup _{0 \leq s \leq t}\left|\varepsilon^{d} \sum_{x} J(\varepsilon x) \eta_{x}(t)-\varepsilon^{d} \sum_{x} J(\varepsilon x) \eta_{x}(0)\right| \geq \delta\right] \\
& \leq 2 \exp \left[2 C(J) \varepsilon^{-1-d} t-\delta \varepsilon^{-d}\right]
\end{aligned}
$$

Let $t=\varepsilon^{2}$. Then, for $\varepsilon$ sufficiently small, the right hand side is bounded by the quantity $\exp \left(-\delta \varepsilon^{-d} / 2\right)$. We divide the interval $[0, T]$ into $\varepsilon^{-2}$ subintervals of size $\varepsilon^{2}$. We can apply previous argument in every subinterval. Taking the intersection of these events we obtain, for sufficiently small $\varepsilon$,

$$
\begin{aligned}
& P_{\varepsilon}^{\xi}\left[\sup _{0 \leq j \leq T \varepsilon^{-2}} \sup _{j \varepsilon^{2} \leq t \leq(j+1) \varepsilon^{2}}\left|\varepsilon^{d} \sum_{x} J(\varepsilon x) \eta_{x}(t)-\varepsilon^{d} \sum_{x} J(\varepsilon x) \eta_{x}\left(j \varepsilon^{2}\right)\right| \geq \delta\right] \\
& \quad \leq T \varepsilon^{-2} \exp \left(-\delta \varepsilon^{-d} / 2\right) \\
& \quad \leq \exp \left(-\delta \varepsilon^{-d} / 8\right)
\end{aligned}
$$

which establishes (4.3).

Equation (4.3) provides some continuity. Hence in order to prove Lemma 3.1, we can assume that the time $t$ and $s$ in (3.1) are of the form $\varepsilon^{2} j$ for some integer $j$. Hence it suffices to prove

LEMMA 4.2 For $\delta$ small enough,

$$
P_{\varepsilon}^{\xi}\left[\sup _{\substack{\varepsilon^{2}|i-j| \leq \delta^{4} \\ 0 \leq i, j \leq T \varepsilon-2}}\left|\varepsilon^{d} \sum_{x} J(\varepsilon x) \eta_{x}\left(\varepsilon^{2} i\right)-\varepsilon^{d} \sum_{x} J(\varepsilon x) \eta_{x}\left(\varepsilon^{2} j\right)\right| \geq \delta\right] \leq \exp \left(-C \varepsilon^{-d}\right)
$$

here $i$ and $j$ are integers and $C$ is some positive constant. 
Proof. From Lemma 3.6, we only have to prove (4.4) for the equilibrium process as long as the constant $C$ can be chosen arbitrary large. From the definition of $Z(\beta, t)$ and Schwarz's inequality, we have for any $t$ fixed and $\beta$ positive,

$$
\begin{aligned}
& E^{\mu_{\varepsilon}} \exp \left[\frac{\beta}{2}\left(\sum_{x} J(\varepsilon x) \eta_{x}(t)-\sum_{x} J(\varepsilon x) \eta_{x}(0)\right)\right] \\
& \leq\left\{E^{\mu_{\varepsilon}}\left[e^{Z(\beta, t)}\right]\right\}^{1 / 2}\left\{E^{\mu_{\varepsilon}}\left[\exp \left[\int_{0}^{t}(\beta U(\eta(s))+\Omega(\eta(s))) d s\right]\right]\right\}^{1 / 2}
\end{aligned}
$$

From the exponential martingale $E^{\mu_{\varepsilon}}\left[e^{Z(\beta, t)}\right]=1$. We can bound $\Omega$ by (4.2). Also, from Lemma 3.7 we have

$$
\varepsilon^{d} \log E^{\mu_{\varepsilon}}\left[\exp \left\{\int_{0}^{t} \beta \varepsilon^{-d} U(\eta(s)) d s\right\}\right] \leq t \sup \operatorname{spec}\left[\beta U(\eta)-\epsilon^{-2} A v_{b}\left(-\mathcal{L}_{b}\right)\right]
$$

The current (3.4) satisfies an identity $(b=(x, x+e))$

$$
\int f w_{x, x+e} d \mu_{\varepsilon}=\int\left[f\left(\eta^{b}\right)-f(\eta)\right] c_{b}(\eta)\left(\eta_{x+e}-\eta_{x}\right) d \mu_{\varepsilon}
$$

Hence by and Schwarz's inequality, we have for any function $f$,

$$
\begin{aligned}
\varepsilon^{-1} \beta \int f w_{x, x+e} d \mu_{\varepsilon} & \leq\left(\varepsilon^{-2} / 4\right) \int c_{b}(\eta)\left[\sqrt{f}\left(\eta^{b}\right)-\sqrt{f}(\eta)\right]^{2} d \mu_{\varepsilon} \\
& +4 \beta^{2} \int\left[\sqrt{f}\left(\eta^{b}\right)+\sqrt{f}(\eta)\right]^{2} c_{b}(\eta)\left(\eta_{x}-\eta_{x+e}\right)^{2} d \mu_{\varepsilon}
\end{aligned}
$$

The last term is bounded by $C \int f d \mu$. Hence, for the eigenvalue problem, we get the bound $C t \beta^{2} \varepsilon^{-d}$. Therefore, together with the bound of $\Omega$ in (4.2) the last expectation in (4.5) is bounded by $C t \beta^{2} \varepsilon^{-d}$. From Chebyshev's inequality,

$$
P^{\mu_{\varepsilon}}\left[\left|\varepsilon^{d} \sum_{x} J(\varepsilon x) \eta_{x}(t)-\varepsilon^{d} \sum_{x} J(\varepsilon x) \eta_{x}(0)\right| \geq \delta\right] \leq \exp \left(-\delta \beta \varepsilon^{-d} / 4+C t \beta^{2} \varepsilon^{-d}\right)
$$

Let $\beta=\delta^{-2}$. For any $t \leq \delta^{4}$ we have

$$
P^{\mu_{\varepsilon}}\left[\left|\varepsilon^{d} \sum_{x} J(\varepsilon x) \eta_{x}(t)-\varepsilon^{d} \sum_{x} J(\varepsilon x) \eta_{x}(0)\right| \geq \delta\right] \leq \exp \left(-\delta^{-1} \varepsilon^{-d} / 8\right)
$$

Clearly, (4.4) follows from this estimate and we conclude Lemma 3.1.

5. The energy estimate. Let us first recall a lemma concerning perturbation theory of eigenvalues applied to our setting. Because we are interested in lattice gases in a finite volume, it is only ordinary perturbation theory for eigenvalues of matrices and the following lemma can be proved by writing down the usual perturbation expansion for eigenvalues and using the assumption on the spectral gap. This lemma actually holds in considerable generality [RS] and an elementary proof is given in [JY].

LEMMA 5.1 Let $\nu$ be a probability measure on a finite set B. Denote by $\langle$, the inner product in $L^{2}(\nu)$ defined by $\langle f, g\rangle=E^{\nu}[f g]$. Let $\mathcal{L}$ be the generator of 
a Markov process on $B$, which is symmetric with respect to $\nu$. Then $A=-\mathcal{L}$ is a nonnegative definite symmetric operator on $L^{2}(\nu)$. Suppose that the lowest eigenvalue 0 of $A$, with the corresponding eigenvector (the ground state) $g_{0} \equiv 1$ has a spectral gap of $\delta>0$. Let $X$ be a bounded function on $B$ considered as a multiplication operator on $L^{2}(\nu)$. Let $\|X\|_{\infty}$ denote the sup norm of $X$. Assume that $E^{\nu}\{X\}=\langle 1, X\rangle=0$. Then there is a universal constant $C$ such that for any constant $\gamma>0$ satisfying

$$
\gamma\|X\|_{\infty} \leq \frac{\delta}{4}
$$

we have

$$
\sup \operatorname{spec} X-A \leq C \gamma^{2}\left\langle X, A^{-1} X\right\rangle+C \gamma^{3} \frac{\|X\|_{\infty}^{3}}{\delta^{2}}
$$

In particular,

$$
\sup \operatorname{spec} X-A \leq C \gamma^{2} \frac{\|X\|_{\infty}^{2}}{\delta}
$$

We can now state the following corollary of Lemma 5.1 in our context. Let $\mu_{\ell, y, \omega}$ denote the (canonical) Gibbs measure with density $y$ and a fixed boundary condition $\omega$ on a cube $\Lambda_{\ell}$ of width $\ell$. Recall the definition of $\mathcal{L}_{b}$ given in (2.12) and (2.13) representing a jump across the bond $b$. The rates are determined in such a way that $\mathcal{L}_{b}$ is symmetric with respect to the measure $\mu_{\ell, y, \omega}$. If the bond $b$ is close to the boundary, the explicit coefficients of $\mathcal{L}_{b}$ depend on the boundary condition. The boundary condition will not be specified because our results are all uniform w.r.t. the boundary conditions. In order to apply Lemma 5.1, we will need an estimate on the spectral gap. A key analytical input is provided by a result from [LY] stating that the gap of the operator $\sum_{b \in \Lambda_{\ell}}\left(-\mathcal{L}_{b}\right)$ is of order $\ell^{-2}$.

LEMMA $5.2 \quad$ Suppose $h$ is a local function in $\mathcal{G}$ (3.7). Let $\ell_{1}=\ell-\sqrt{\ell}$ and $V_{\ell}(h, y)$ be given by (3.10). Recall also that the mixing condition Assumption $(A)$ is satisfied. Then there is a universal constant $C$ such that for any $\gamma>0$ we have the following estimate on the eigenvalue

$$
\sup \operatorname{spec}\left[\epsilon^{-1} A v_{|x| \leq \ell_{1}} h-\gamma \epsilon^{-2} A v_{b \in \Lambda_{\ell}}\left(-\mathcal{L}_{b}\right)-C V_{\ell}(h, y)\right] \leq 0
$$

provided

$$
\varepsilon \ll 1, \quad \ell \gg 1 \text { and } \quad \ell \epsilon^{\frac{1}{d+2}} \ll 0
$$

Furthermore,

$$
V_{\ell}(h, y) \leq C \ell^{d+2} E^{\mu_{\ell, y, \omega}}\left[\left(A v_{|x| \leq \ell_{1}} \tau_{x} h\right)^{2}\right]
$$

Proof. From [LY], the spectral gap of the operator $\epsilon^{-2} A v_{b \in \Lambda_{\ell}}\left(-\mathcal{L}_{b}\right)$ is of order $\epsilon^{-2} \ell^{-2-d}$. It is easy to verify that (5.3) implies the condition (5.1). Hence the supnorm of the first term in (5.2) which is of order $\epsilon^{-1}$ is much smaller than the spectral gap of the operator appearing as the second term in (5.2) provided (5.3) is satisfied. Hence (5.2) is just a simple consequence of Lemma 5.1. The bound (5.4) just restates that the spectral gap is of order $\ell^{-2}$.

The following theorem combines Lemma 5.2 with multi-scale analysis and provides a key estimate needed in estimating eigenvalues in connection with Theorem 3.9 and the energy estimate Lemma 3.2. 
Let us recall that $\Lambda_{\varepsilon^{-1}}$ is the periodic lattice of side $\varepsilon^{-1}$ and $\mu_{\varepsilon}$ is the Gibbs measure on it. For any $k, \mathcal{F}_{k}$ is the $\sigma$-field generated by the configuration in $\Lambda_{k}^{c}$ i.e. $\left\{\eta_{x}: x \in \Lambda_{k}^{c}\right\}$ and the density $\bar{\eta}_{k}=A v_{\Lambda_{k}} \eta_{x}$ in $\Lambda_{k}$. The complement $\Lambda_{k}^{c}$ is relative to the periodic lattice $\Lambda_{\varepsilon^{-1}}$ in this context. Otherwise it could be with respect to $Z^{d}$.

THEOREM 5.3 Let $h$ be a local function satisfying

$$
\begin{aligned}
\sup _{\eta}|h(\eta)| & \leq C_{h} \\
\sup _{\eta}\left|E^{\mu_{\varepsilon}}\left[h \mid \Lambda_{k}^{c}, \bar{\eta}_{k}\right]\right| & \leq C_{h} k^{-d / 2-1-2 \alpha}
\end{aligned}
$$

with constants $C_{h}$ and $\alpha>0$ and for all $k$ in the range $1 \leq k \leq \epsilon^{-\frac{2}{2+d}}$. Then there are positive constants $C, \kappa$ and a function $F$ such that for any $h, \gamma>0$,

$$
\sup \operatorname{spec}\left[\epsilon^{-1} h-\gamma \epsilon^{-2} \sum_{b \in \Lambda_{\varepsilon^{-1}}}\left(-\mathcal{L}_{b}\right)|b-0|^{-\alpha-d}\right] \leq C \gamma^{-1} C_{h}^{2}+\varepsilon^{\kappa} F\left(\gamma, C_{h}\right)
$$

on $L^{2}\left(\mu_{\varepsilon}\right)$ where $\mu_{\varepsilon}$ is the Gibbs measure defined in section 2. Furthermore, (5.5) is satisfied for all $h \in \mathcal{G}$, as well as for $h=w_{x, x+e}, h=\nabla_{e} \eta_{x}$ and $h=\mathcal{L} g$ for some local function $g$.

Let a nonlocal function $h^{\prime}$ be of the special form $h^{\prime}(\eta)=G h$, where, for some $\delta>0, G=G\left(\eta, \bar{\eta}_{\delta \varepsilon^{-1}}\right)$ depends only on the configuration $\eta \mid \Lambda_{\delta \varepsilon^{-1}}^{c}$ and the density $\bar{\eta}_{0, \delta \varepsilon^{-1}}$ and $h$ is a local function satisfying (5.5). Then (5.6) remains valid in a slightly modified form

$$
\sup \operatorname{spec}\left[\epsilon^{-1} h^{\prime}-C_{0} \gamma^{-1} G^{2}-\gamma \epsilon^{-2} \sum_{b \in \Lambda_{\varepsilon^{-1}}}\left(-\mathcal{L}_{b}\right)|b-0|^{-\alpha-d}\right] \leq \varepsilon^{\kappa} F\left(\gamma, C_{G}\right)
$$

where $C_{0}$ is a universal constant and $C_{H}$ is the $L_{\infty}$ bound for $G$.

If we replace $h$ by $h_{s}=E^{\mu}\left[h \mid \mathcal{F}_{0, s}\right]$, then for any $\gamma>0$,

$$
\underset{s \rightarrow \infty}{\limsup } \limsup _{\varepsilon \rightarrow 0} \sup \operatorname{spec}\left[\varepsilon^{-1} h_{s}-\gamma \varepsilon^{-2} \sum_{b}\left(-\mathcal{L}_{b}\right)|b-x|^{-\alpha-d}\right] \leq 0
$$

REMARK. For the rest of this paper, the constant $\alpha$ will be fixed and can be chosen to be any small positive number.

Proof of Part I. Fix an integer $\ell$. Let us denote by $\mathcal{F}_{n}$ the $\sigma$-field $\mathcal{F}_{\ell^{n}}$ and by $\Lambda_{n}$ the cube $\Lambda_{\ell^{n}}$. Define $h_{n}$ by

$$
h_{n}=E^{\mu_{\varepsilon}}\left[h \mid \mathcal{F}_{n}\right]
$$

as the conditional expectation of $h$ w.r.t. $\mathcal{F}_{n}$. By definition, $h_{0}=h$. Rewrite the function $h$ appearing on the left side of (5.7) as

$$
h=\sum_{n=0}^{M-1}\left(h_{n}-h_{n+1}\right)+h_{M}
$$

where $M$ is the largest integer such that $\ell^{M[(d / 2)+1]} \leq(q \varepsilon)^{-1}$, so that

$$
\ell^{M[(d / 2)+1]} \leq(q \varepsilon)^{-1} \leq \ell^{(M+1)[(d / 2)+1]}
$$


with a constant $q$ to be determined later. From the assumption (5.5) we have

$$
\sup _{\eta}\left|h_{M}(\eta)\right| \leq C_{h} \ell^{-M(d / 2+1+2 \alpha)}
$$

With (5.11),

$$
\varepsilon^{-1} \sup _{\eta}\left|h_{M}(\eta)\right| \leq C_{h} \ell^{(d / 2+1+2 \alpha)} \varepsilon^{-1}(q \varepsilon)^{\kappa}
$$

where $\kappa=1+\frac{4 \alpha}{2+d}>1$.

We compare the two sides of (5.13) in operator sense. For some $C<\infty$,

$$
\sum_{n} k^{-\alpha} A v_{b \in \Lambda_{n+1}}\left(-\mathcal{L}_{b}\right) \leq C \sum_{b}\left(-\mathcal{L}_{b}\right)|b-0|^{-\alpha-d}
$$

where $k=\ell^{n+1}$. Because each $-\mathcal{L}_{b}$ is a symmetric positive semidefinite operator establishing (5.13) amounts to verifying

$$
\sum_{n: \Lambda_{n+1} \ni b} k^{-\alpha-d} \leq C \sum_{b}|b-0|-\alpha-d
$$

for all $b$. This is easily seen to be true with a constant $C<\infty$, that depends only on $\ell$ and $\alpha$. We can clearly estimate the term on the left hand side of (5.6) by decomposing both the function $h$ and the operator according to (5.10) and (5.13) to obtain

$$
\begin{aligned}
& \operatorname{supspec}\left[\epsilon^{-1} h-\gamma \epsilon^{-2} \sum_{b \in \Lambda_{\epsilon^{-1}}}\left(-\mathcal{L}_{b}\right)|b-0|^{-\alpha-d}\right] \\
& \leq \operatorname{supspec}\left[\epsilon^{-1} h-\frac{\gamma}{C} \epsilon^{-2} \sum_{n} k^{-\alpha} A v_{b \in \Lambda_{n+1}}\left(-\mathcal{L}_{b}\right)\right] \\
& \leq \sum_{n=0}^{M-1} \mathcal{E}_{n+1}+\sup _{\eta}\left|h_{M}(\eta)\right|
\end{aligned}
$$

where

$$
\mathcal{E}_{n+1}=\sup \operatorname{spec}\left[\varepsilon^{-1}\left(h_{n}-h_{n+1}\right)-\frac{\gamma}{C} \epsilon^{-2} k^{-\alpha} A v_{b \in \Lambda_{n+1}}\left(-\mathcal{L}_{b}\right)\right]
$$

By limiting the operator to bonds in some $\Lambda_{n+1}$ we have introduced considerable degeneracy. The extremal invariant measures are precisely the canonical Gibbs measures $\mu_{n+1}$ in the box $\Lambda_{n+1}$ and are parameterized by $\omega$, representing the boundary condition on $\Lambda_{n+1}^{c}$ and the average density in $\Lambda_{n+1}$. Since the spectrum is calculated in each $L_{2}\left(\mu_{n+1}\right)$ we obtain an upper bound for $\mathcal{E}_{n+1}$ by estimating it in each $L_{2}\left(\mu_{n+1}\right)$ and then taking supremum over $\omega$. Hence we only need to get bounds on (5.16) in $L_{2}\left(\mu_{n+1}\right)$ that are uniform in $\omega$. Note that once $\mathcal{F}_{n+1}$ is given, from [LY] the spectral gap of $\frac{\gamma}{C} \epsilon^{-2} k^{-\alpha} A v_{b \in \Lambda_{n+1}}\left(-\mathcal{L}_{b}\right)$ is bounded below by $\frac{c \gamma}{C} \epsilon^{-2} k^{-2-d-\alpha}$ for some $c>0$. We note in addition that $\int\left(h_{n}-h_{n+1}\right) d \mu_{n+1}=0$. For the perturbation theory to work, by Lemma 5.1 , we need

$$
\epsilon^{-1}\left\|h_{n}-h_{n+1}\right\|_{\infty} \leq \frac{c \gamma}{4 C} \epsilon^{-2} \ell^{-(n+1)(2+d+\alpha)}
$$


to hold for all $0 \leq n \leq M-1$. From our assumption (5.5) we see that

$$
\varepsilon^{-1}\left\|h_{n}-h_{n+1}\right\|_{\infty} \leq 2 C_{h} \varepsilon^{-1} \ell^{-n(d / 2+1+2 \alpha)}
$$

so that (5.17) holds provided

$$
2 C_{h} \varepsilon^{-1} \ell^{-n(d / 2+1+2 \alpha)} \leq \frac{c \gamma}{4 C} \epsilon^{-2} \ell^{-(n+1)(2+d+\alpha)}
$$

which is implied by

$$
\ell^{M(d / 2+1)} \leq \frac{c \gamma}{8 C C_{h}} \varepsilon^{-1}
$$

so that the choice of $q=\max \left(1, \frac{8 C C_{h}}{c \gamma}\right)$ will work in (5.11) as well as (5.18). By Lemma 5.2 one can now bound $\mathcal{E}_{n+1}$ by

$$
\begin{aligned}
\mathcal{E}_{n+1} & \leq C \gamma^{-1} \epsilon^{-2} \epsilon^{2} k^{d+2+\alpha}\left\|h_{n}-h_{n+1}\right\|_{\infty}^{2} \\
& \leq C \gamma^{-1} k^{-\alpha} C_{h}^{2}=C \gamma^{-1} C_{h}^{2} \ell^{-\alpha(n+1)}
\end{aligned}
$$

We have denoted by $C$, a constant that may change from line to line, but will always remain independent of the parameters $\varepsilon, \gamma$ and $h$.. Summing over $n$, we conclude that

$$
\begin{aligned}
\sum_{n=0}^{M-1} \mathcal{E}_{n+1}+\sup _{\eta}\left|h_{M}(\eta)\right| & \leq C \gamma^{-1} C_{h}^{2}+C C_{h} \varepsilon^{\frac{4 \alpha}{2+d}}\left(1+\frac{8 C C_{h}}{c \gamma}\right)^{\left(1+\frac{4 \alpha}{2+d}\right)} \\
& =C \gamma^{-1} C_{h}^{2}+\varepsilon^{\kappa} F\left(\gamma, C_{h}\right)
\end{aligned}
$$

concluding the proof of part I of Theorem 5.3.

Proof of Part II. Clearly, (5.5) holds for $h \in \mathcal{G}$. From (4.7) $w_{x, x+e} \in \mathcal{G}$ and (5.5) holds. For any local function $g$, there is an $s$ large enough such that $g$ is measurable w.r.t. the $\sigma$-algebra generated by $\left\{\eta_{x},|x| \leq s\right\}$. Let $\mathcal{L}_{s}=\sum_{b \in \Lambda_{s}} \mathcal{L}_{b}$. Then we have $\mathcal{L} g=\mathcal{L}_{s} g$ and also $E^{\mu_{\varepsilon}}\left[\mathcal{L}_{s} g \mid \mathcal{F}_{s}\right]=0$. In other words, $\mathcal{L} g \in \mathcal{G}$. This proves (5.5) when $h=\mathcal{L} g$. We now establish (5.5) for $h=\nabla_{e} \eta_{x}$, namely,

$$
E^{\mu_{\varepsilon}}\left[\nabla_{e} \eta_{0} \mid \mathcal{F}_{k}\right] \leq C k^{-d / 2-1-\alpha} .
$$

This is clearly a type of mixing condition. Unfortunately, the mixing condition we have is for the grand canonical measure (2.5) rather than the canonical one that we are interested in here. We shall however prove in Section 10, that (5.20) holds .

We now prove (5.7) for $h^{\prime}=G h$. Note that in the argument in the part I, $G$ is effectively a constant and replacing $h$ by $G h$ only changes $C_{h}$ into $G C_{h}$. Therefore (5.7) is not all that different from (5.6). Finally to see (5.8) we can assume for simplicity that $l^{n_{0}}=s$ and sum (5.17) for $n \geq n_{0}$. This concludes the proof of Theorem 5.3.

Lemma 5.3 states that, in an average sense, any $h_{x}$ satisfying (5.5) $E^{f}\left\{\epsilon^{-1} h_{x}\right\}$ is of order 1 for any probability density $f$ satisfying

$$
\epsilon^{-2} A v_{b \in \Lambda} D_{b}(\sqrt{f}) \leq C
$$

We now prove the energy estimate.

Proof of Lemma 3.2. As we have already established tightness of the empirical measure valued process $\varepsilon^{d} \sum_{x} \delta_{\varepsilon x} \eta_{x}(\cdot)$, we know that any limit point of $Q^{\varepsilon}$ is a measure $Q$ on the space $C[0, T] ; \mathcal{M}]]$ of weakly continuos maps $t \rightarrow m(t, \theta)$ of densities on $\mathbf{T}^{d}$ 
that are bounded by 1 . The energy estimate is established by proving, for some finite $C$

$$
E^{Q}\left\{\sup _{J}\left[\int_{0}^{T} \int_{\mathbf{T}^{d}}\left(D_{e} J\right)(t, \theta) m(t, \theta) d t d \theta-\frac{C}{2} \int_{0}^{T} \int_{\mathbf{T}^{d}}|J(t, \theta)|^{2} d t d \theta\right]\right\} \leq C
$$

where the supremum is taken over all smooth functions of $t$ and $\theta$. If we ignore the supremum, for the approximating processes, this amounts to proving

$$
\limsup _{\varepsilon \rightarrow 0} E^{Q_{\varepsilon}}\left\{\varepsilon^{d} \int_{0}^{T} \sum_{x}\left(\nabla_{e} J\right)\left(t, \frac{x}{N}\right) \eta_{x}(t) d t-\frac{C}{2} \varepsilon^{d} \int_{0}^{T} \sum_{x}\left|J\left(t, \frac{x}{N}\right)\right|^{2} d t\right\} \leq C
$$

or

$$
\limsup _{\varepsilon \rightarrow 0} E^{Q_{\varepsilon}}\left\{\varepsilon^{d-1} \int_{0}^{T} \sum_{x} J\left(t, \frac{x}{N}\right) \nabla_{e} \eta_{x}(t) d t-\frac{C}{2} \varepsilon^{d} \int_{0}^{T} \sum_{x}\left|J\left(t, \frac{x}{N}\right)\right|^{2} d t\right\} \leq C
$$

In view of Jensen's inequality and the relative entropy bound $H\left(Q^{\varepsilon} ; P^{\varepsilon}\right) \leq C \varepsilon^{-d}$, it is sufficient to establish

$$
\begin{gathered}
\limsup _{\varepsilon \rightarrow 0} \varepsilon^{d} \log E^{P_{\varepsilon}}\left\{\operatorname { e x p } \left[\varepsilon^{-1} \int_{0}^{T} \sum_{x} J\left(t, \frac{x}{N}\right) \nabla_{e} \eta_{x}(t) d t\right.\right. \\
\left.\left.-\frac{C}{2} \int_{0}^{T} \sum_{x}\left|J\left(t, \frac{x}{N}\right)\right|^{2} d t\right]\right\} \leq 0
\end{gathered}
$$

or equivalently

$$
\limsup _{\varepsilon \rightarrow 0} \varepsilon^{d} \log E^{P_{\varepsilon}}\left\{\exp \left[\varepsilon^{-1} \int_{0}^{T} \sum_{x} J\left(t, \frac{x}{N}\right) \nabla_{e} \eta_{x}(t) d t\right]\right\} \leq \frac{C}{2} \int_{0}^{T} \int_{\mathbf{T}^{d}}|J(t, \theta)|^{2} d t d \theta
$$

Since $P^{\varepsilon}$ is the equilibrium and the functional inside the expectation is a FeynmanKac functional with a time dependent potential we can estimate (5.26) by estimating the largest eigenvalue. If we denote by

$$
\lambda_{\varepsilon}(t)=\sup \operatorname{spec}\left[\varepsilon^{-1} \sum_{x} J\left(t, \frac{x}{N}\right) \nabla_{e} \eta_{x}-\varepsilon^{-2} \sum_{b}\left(-\mathcal{L}_{b}\right)\right]
$$

it is sufficient to prove

$$
\limsup _{\varepsilon \rightarrow 0} \varepsilon^{d} \int_{0}^{T} \lambda_{\varepsilon}(t) d t \leq \frac{C}{2} \int_{0}^{T} \int_{\mathbf{T}^{d}}|J(t, \theta)|^{2} d t d \theta
$$

For estimating the eigenvalue $\lambda_{\varepsilon}$ we decompose

$$
\varepsilon^{-1} \sum_{x} J\left(t, \frac{x}{N}\right) \nabla_{e} \eta_{x}-\varepsilon^{-2} \sum_{b}\left(-\mathcal{L}_{b}\right)
$$

and, for some $\gamma>0$, estimate $\varepsilon^{d} \lambda_{\varepsilon}(t)$ from above by the sum

$$
\varepsilon^{d} \lambda_{\varepsilon}(t) \leq \varepsilon^{d} \sum_{x} \sup \operatorname{spec}\left[\varepsilon^{-1} J\left(t, \frac{x}{N}\right) \nabla_{e} \eta_{x}-\gamma \sum_{b}|b-x|^{-\alpha-d}\left(-\mathcal{L}_{b}\right)\right]
$$


We have in addition the bound

$$
\begin{aligned}
\sup _{0 \leq t \leq T} \varepsilon^{d} \lambda_{\varepsilon}(t) & \leq \sup _{0 \leq t \leq T} \varepsilon^{d} \sum_{x} \sup \operatorname{spec}\left[\varepsilon^{-1} J\left(t, \frac{x}{N}\right) \nabla_{e} \eta_{x}-\gamma \sum_{b}|b-x|^{-\alpha-d}\left(-\mathcal{L}_{b}\right)\right] \\
& \leq \sup _{|\beta| \leq C} \sup _{x} \sup \operatorname{spec}\left[\varepsilon^{-1} \beta \nabla_{e} \eta_{x}-\gamma \sum_{b}|b-x|^{-\alpha-d}\left(-\mathcal{L}_{b}\right)\right]
\end{aligned}
$$

where $C$ is an upper bound on $|J(\cdot, \cdot)|$. Now (5.6) provides a uniform bound

$$
\sup _{0 \leq t \leq T} \varepsilon^{d} \lambda_{\varepsilon}(t) \leq C
$$

as well as a bound on the limit

$$
\lim _{\varepsilon \rightarrow 0} \varepsilon^{d} \int_{0}^{T} \lambda_{\varepsilon}(t) d t \leq \frac{C}{2} \int_{0}^{T}|J(t, \theta)|^{2} d t d \theta
$$

concluding our estimate. In order to complete the proof of Lemma 3.2 we only have to remark that whenever we use large deviation bounds and Jensen's inequality to pass from

$$
\limsup _{\varepsilon \rightarrow 0} \varepsilon^{d} \log E^{P^{\varepsilon}}\left\{\exp \left[\varepsilon^{-d} F_{\alpha}\right]\right\} \leq 0
$$

and

$$
H\left(Q^{\varepsilon} ; P^{\varepsilon}\right) \leq C \varepsilon^{-d}
$$

to

$$
E^{Q}\left[F_{\alpha}\right] \leq C
$$

we actually can obtain

$$
E^{Q}\left[\sup _{\alpha} F_{\alpha}\right] \leq C
$$

because, while one cannot sneak the supremum inside the expectation to go from (5.29) to (5.30), to do it in (5.28) is trivial and for any finite set of $\alpha$ 's yields (5.30) with the same constant $C$, independently of the finite set. The rest is a routine application of the monotone convergence theorem.

6. Two blocks estimate. In this section, we prove the two block estimate Theorem 6.2. It will be used in the next section to prove Theorem 3.9. The first step toward proving a two block estimate is to get a bound on the Dirichlet form involving "long jumps" in terms of the usual Dirichlet form with only the "nearest-neighbor jumps". This was one of the main difficulties in establishing the hydrodynamical limit of lattice gas models in dimension greater than 1 . If $\mu$ is a Gibbs state on the space of configurations on a lattice $\Lambda$ and $z, y$ are two lattice sites that are a considerable distance away from each other, the problem is one of estimating $E^{\mu}\left\{\left|\left(T_{z, y} u\right)(\eta)\right|^{2}\right\}=$ $E^{\mu}\left\{\left|u\left(\eta^{z, y}\right)-u(\eta)\right|^{2}\right\}$ in terms of the usual Dirichlet form involving $E^{\mu}\left\{\mid u\left(\eta^{x, x+e}\right)-\right.$ $\left.\left.u(\eta)\right|^{2}\right\}$. Let us introduce the notation $S_{x, y} \eta=\eta^{x, y}$. The natural way to make this estimation is to write

$$
\begin{aligned}
S_{z, y} & =S_{z, x_{1}} S_{x_{1}, x_{2}} \cdots S_{x_{n-1}, x_{n}} S_{x_{n}, y} S_{x_{n-1}, x_{n}} \cdots S_{x_{1}, x_{2}} S_{z, x_{1}} \\
& =S_{b_{2 n-1}} \cdots S_{b_{1}}
\end{aligned}
$$


as a product of exchanges over shorter bonds and estimate $T_{x, y} u$ by a telescoping sum

$$
\left|u\left(S_{x, y} \eta\right)-u(\eta)\right| \leq \sum_{j=1}^{2 n-1}\left|u\left(S_{b_{j}} \cdots S_{b_{1}} \eta\right)-u\left(S_{b_{j-1}} \cdots S_{b_{1}} \eta\right)\right|
$$

and conclude that

$$
\begin{aligned}
E^{\mu}\left\{\left|u\left(S_{x, y} \eta\right)-u(\eta)\right|^{2}\right\} & \leq(2 n-1) \sum_{j=1}^{2 n-1} E^{\mu}\left\{\left|u\left(S_{b_{j}} \cdots S_{b_{1}} \eta\right)-u\left(S_{b_{j-1}} \cdots S_{b_{1}} \eta\right)\right|^{2}\right\} \\
& =(2 n-1) \sum_{j=1}^{2 n-1} E^{\mu}\left\{\left|u\left(S_{b_{j}} S^{j} \eta\right)-u\left(S^{j} \eta\right)\right|^{2}\right\}
\end{aligned}
$$

where $S^{j}=S_{b_{j}} \cdots S_{b_{1}}$ is some more complex permutation along the way. If $\mu$ were a Bernoulli measure or some other permutation invariant measure like a Bernoulli measure conditioned on the value of the sum $\sum_{x} \eta_{x}$ then

$$
(2 n-1) \sum_{j=1}^{2 n-1} E^{\mu}\left\{\left|u\left(S_{b_{j}} S^{j} \eta\right)-u\left(S^{j} \eta\right)\right|^{2}\right\}=(2 n-1) \sum_{j=1}^{2 n-1} E^{\mu}\left\{\left|u\left(S_{b_{j}} \eta\right)-u(\eta)\right|^{2}\right\}
$$

and, if we choose our intermediary chain of sites to be nearest neighbors, we are done. Difficulties arise because, in general, $\mu$ is not invariant under permutation. We will pickup a constant factor which is an upper bound on the Radon-Nikodym derivatives when $\mu$ is transformed by the permutation $S^{j}$. We note that if we are able to bound the Radon-Nikodym derivatives in both directions then conditioning to a set of the form $\sum_{x} \eta_{x}=m$ causes no problem. While the factor $2 n-1$ which is comparable to the distance from $x$ to $y$ is acceptable, if not taken care of, the bound for the Radon Nikodym derivative will grow exponentially in $n$ and is unacceptable for our purposes. The following theorem therefore requires a careful proof. Without loss of generality we will assume that the jump rate $c_{b}=1$ in (2.12).

Theorem 6.1 Denote by $\mu$ a Gibbs state on a cube $\Lambda$ and let $D_{b}$ denote the Dirichlet form w.r.t $\mu$ for a nearest neighbor bond $b \in \Lambda$. For any two sites $x, y \in \Lambda$ let $\gamma_{x y}$ denote the nearest neighbor path that goes from $x$ to $y$, moving successively as far as it has to in each of the coordinate directions, following the natural order for the different coordinate directions. For every function $u$ on $\{0,1\}^{|\Lambda|}$ define the Dirichlet form along the path by

$$
D^{\gamma_{z y}}(u)=\sum_{b \in \gamma_{x y}} D_{b}(u)
$$

Then we have

$$
E^{\mu}\left[\left(T_{z y} u\right)^{2}\right] \leq c_{0}\left|\gamma_{z y}\right| D^{\gamma_{z y}}(u),
$$

where $c_{0}$ is a constant depending on the dimension $d$ and the Hamiltonian. Here $\left|\gamma_{z y}\right|$ is the length of the path. Note that no mixing condition is needed.

Proof. Step 1. With out loss of generality we can assume that $y=z+\ell e_{1}$. The general path is just a finite sequence of such straight lines $\left(z, x_{1}\right),\left(x_{1}, x_{2}\right) \cdots\left(x_{d-1}, y\right)$ along different coordinate directions going through at most $d-1$ intermediary corners $x_{1}, \cdots, x_{d-1}$. We can estimate the left side of (6.1) by a telescoping sum. Although 
the Radon-Nikodym derivatives could cause trouble here, because the only transformations that are involved in the telescoping sum are permutations among the $d+1$ sites $z, x_{1}, \cdots, x_{d-1}, y$ the extra factor is just a constant depending on the Hamiltonian and $d$.

Step 2. We can assume with out loss of generality that $y=z+\ell k e_{1}$ for some $\ell$ with $k$ fixed at some positive integer so that two sites that are at a distance at least $k$, do not both interact with any third site in the Hamiltonian. This involves again the same idea. If $z-y=\ell e_{1}$ where $\ell$ is not a multiple of $k$, we can go through an intermediate point that is a multiple and the difference is at most a fixed finite distance away.

Step 3. The choice of $k$ in the previous step guarantees that the conditional distribution of $\left\{\eta_{z+j k e_{1}}: 0 \leq j \leq \ell\right\}$ under the canonical Gibbs measure given the configuration on the complement of the sites $\left\{z+j k e_{1}\right\}$ is a product measure conditioned on the sum $\sum_{j=1}^{\ell} \eta_{z+j k e_{1}}$. Of course the conditional probability $p_{\omega}(j)=\mu_{\omega}\left[\eta_{z+j k e_{1}}=1\right]$ is given by the DLR formula, and although different for each $j$ takes only a fixed finite number of values depending on the total number of different configurations possible in the neighborhood of any one site. If we can establish (6.1) for the conditional distributions $\mu_{\omega}$ with a uniform constant independent of $\omega$, we can integrate with respect to $\mu$ at the end.

Step 4. The problem is now reduced to the following. We just have sites $0,1 \cdots, \ell$. We have a measure $\mu$ which is the product measure with $\operatorname{Pr}\left[\eta_{j}=1\right]=p_{j}$ conditioned on the set $\sum_{j=0}^{\ell} \eta_{j}=m$. If we assume that $\left\{p_{j}\right\}$ takes only a finite number $r$ of distinct values and they all satisfy $\delta \leq p_{j} \leq 1-\delta$, then with a constant $c_{0}$ depending only on $r$ and $\delta$, the analog of (6.1) is satisfied. This problem came up in the context of one dimensional lattice gases with random magnetization and the proof given in [QY1] is reproduced below. The proof is by induction on $r$ where $r$ is redefined as the number of distinct values among $\left\{p_{j}\right\}$ with $1 \leq j \leq \ell-1$. If $r=1$, except for the end points all probabilities are the same. In this case the factor produced by the Radon-Nikodym derivative is easily seen to be bounded by a constant independent of $\ell$. Any permutation can only change the probabilities at most four sites. Now let us prove the induction step. Assume that if there are $r$ distinct values we have a bound with a constant $c_{r}$ independent of $\ell$. Suppose now that there are $r+1$ distinct values. Let $1 \leq j_{1}<j_{2}<\cdots<j_{s} \leq \ell-1$ be an enumeration of all the sites where $p_{j}$ is equal to one of these distinct values. If we write

$$
S_{0, \ell}=S_{0, j_{1}} S_{j_{1}, j_{2}} \cdots S_{j_{s-1}, j_{s}} S_{j_{s}, \ell} S_{j_{s-1}, j_{s}} \cdots S_{j_{1}, j_{2}} S_{0, j_{1}}
$$

all but the two endsites have the same probability and therefore, for all the intermediate permutations, the Radon-Nikodym factor is controlled uniformly in $s$ by a constant $C$. By Schwarz's inequality

$$
\begin{aligned}
& \frac{1}{\ell} E^{\mu}\left\{\left|T_{0, \ell} u\right|^{2}\right\} \\
& \quad \leq C\left[\frac{1}{j_{1}} E^{\mu}\left\{\left|T_{0, j_{1}} u\right|^{2}\right\}+\sum_{r=2}^{s} \frac{1}{j_{r}-j_{r-1}} E^{\mu}\left\{\left|T_{j_{r-1}, j_{r}} u\right|^{2}\right\}+\frac{1}{\ell-j_{s}} E^{\mu}\left\{\left|T_{j_{s}, \ell} u\right|^{2}\right\}\right]
\end{aligned}
$$

By the induction hypothesis each summand satisfies the analog of (6.1). We obtain $c_{r+1} \leq C c_{r}$ and the theorem is established.

Using this Theorem, it is not hard to prove the two block estimate. The following proof is similar to the one given in [GPV, R]. 
Theorem 6.2 (Two Blocks Estimate) For any bounded continuous function $h$ and any $\gamma>0$,

$$
\underset{k, a, \varepsilon}{\limsup \sup \operatorname{spec}}\left\{A v_{x} A v_{y:|y-x| \leq a \varepsilon^{-1}}\left[h\left(\bar{\eta}_{x, k}\right)-h\left(\bar{\eta}_{y, k}\right)\right]^{2}-\gamma \epsilon^{-2} A v_{b}\left(-\mathcal{L}_{b}\right)\right\}=0
$$

where $\lim \sup _{k, a, \varepsilon}=\lim \sup _{k \rightarrow \infty} \lim \sup _{a \rightarrow 0} \lim \sup _{\epsilon \rightarrow 0}$. In particular,

$$
\underset{k, a, \varepsilon}{\limsup \sup \operatorname{spec}}\left\{A v_{x}\left[h\left(\bar{\eta}_{x, a \epsilon^{-1}}\right)-h\left(\bar{\eta}_{x, k}\right)\right]^{2}-\gamma \epsilon^{-2} A v_{b}\left(-\mathcal{L}_{b}\right)\right\}=0
$$

Proof. Let $L_{x, y}$ be the generator associated with the Dirichlet form $E^{\mu}\left[\left(T_{x y} u\right)^{2}\right]$, namely they are related via (2.12). For every pair $x, y$ of sites in $\Lambda$ let us choose a canonical path $\gamma_{x, y}$, consisting of $d$ straightlines that lie along the $d$ coordinate directions following their natural order. $\left|\gamma_{x, y}\right|$ is of order $|x-y|$. Recall that $R$ is the range of the Hamiltonian defined in section 2. Hence we can rewrite (6.1) as an operator inequality

$$
-L_{w, z} \leq C|w-z| \sum_{b \in \gamma_{w z}}\left(-\mathcal{L}_{b}\right)
$$

Taking the average of $w$ with $|w-x| \leq k$ and $z$ with $|z-y| \leq k$ and then averaging over $x \in \Lambda$ and $y$ with $2 R+k<|y-x| \leq a \epsilon^{-1}$, we have

$$
A v_{x} A v_{y: 2 R+k<|y-x| \leq a \epsilon^{-1}} A v_{w:|w-x| \leq k} A v_{z:|z-y| \leq k}\left(-\mathcal{L}_{w, z}\right) \leq C \epsilon^{-2} a^{2} A v_{b}\left(-\mathcal{L}_{b}\right)
$$

A similar inequality holds if we replace $|z-y| \leq k$ by $|z-x| \leq k$ or replace $|w-x| \leq k$ by $|w-y| \leq k$. Let $\Lambda_{x}^{k}$ denote a cube of width $k$ and centered at $x$. Hence we have

$$
A v_{x} A v_{y: 2 R+k<|y-x| \leq a \epsilon^{-1}} A v_{w, z \in \Lambda_{x}^{k} \cup \Lambda_{y}^{k}}\left(-\mathcal{L}_{w, z}\right) \leq C \epsilon^{-2} a^{2} A v_{b}\left(-\mathcal{L}_{b}\right)
$$

We can replace the average of $y$ in (6.2) by the average over $y$ with $2 R+k<$ $|y-x| \leq a \epsilon^{-1}$ since the error term is negligible. Together with (6.5), we can bound the eigenvalue of $(6.2)$ by

$$
\begin{aligned}
& A v_{x} A v_{y: 2 R+k<|y-x| \leq a \epsilon^{-1}} \sup \operatorname{spec}\left\{\left[h\left(\bar{\eta}_{x, k}\right)-h\left(\bar{\eta}_{y, k}\right)\right]^{2}\right. \\
& \left.-A v_{w, z \in \Lambda_{x}^{k} \cup \Lambda_{y}^{k}} C a^{-2}\left(-\mathcal{L}_{w, z}\right)\right\}
\end{aligned}
$$

Clearly we obtain an upper bound by replacing the average over $x$ and $y$ with sup over $x$ and $y$ and over the boundary conditions of $\Lambda_{x}^{k} \cup \Lambda_{y}^{k}$. Denote the configuration on $U=\Lambda^{k} \cup \Lambda^{k}$ by $(\xi, \zeta)$. Then (6.6) is bounded above by the supremum of

$$
\sup \operatorname{spec}\left\{\left[h\left(\bar{\xi}_{k}\right)-h\left(\bar{\zeta}_{k}\right)\right]^{2}-C a^{-2} A v_{w, z \in U}\left(-\mathcal{L}_{w, z}\right)\right\}
$$

as an eigenvalue problem on $L^{2}\left(\mu_{U, \omega, \rho}\right)$ with boundary condition $\omega$ and density $\rho$.

We now fix a boundary condition $\omega$ and a density $\rho$. The operator

$$
C a^{-2} A v_{w, z \in U}\left(-\mathcal{L}_{w, z}\right)
$$


on $L^{2}\left(\mu_{U, \omega, \rho}\right)$ clearly has a gap $C(k) a^{-2}$ and the ground state is the constant function. For any $k$ fixed, and $a \rightarrow 0$, the gap diverges to $\infty$. Since $h$ is bounded, for any $k$ fixed from Lemma 5.1 we can bound the limsup of (6.7) as $a \rightarrow 0$ by

$$
\sup _{\omega, \rho} E^{\mu_{U, \omega, \rho}}\left[h\left(\bar{\xi}_{k}\right)-h\left(\bar{\zeta}_{k}\right)\right]^{2}
$$

Taking the limit $k \rightarrow \infty$, we have $\mu_{U, \omega, \rho} \rightarrow \mu_{\rho} \times \mu_{\rho}$, where $\mu_{\rho}$ denotes the infinite volume Gibbs measure on $\mathbb{Z}^{d}$ with density $\rho$. Clearly, (6.2) and (6.3) follow from the law of large number of Gibbs states. This proves Theorem 6.2.

7. Eigenvalue estimate. We are now ready to prove Theorem 3.9.

Proof of Theorem 3.9. Step 1. Let us recall that $\Phi_{x, e}^{\mathbf{g}}$, defined in (3.17), and $\Psi_{\varepsilon, c, e}$ defined in Theorem 3.9 are given by

$$
\begin{aligned}
\Phi_{x, e}^{\mathbf{g}} & =A v_{|y-x| \leq \ell_{1}}\left[w_{y, y+e}+\mathcal{L} g_{e}\left(\bar{\eta}_{x, \ell}, \tau_{y} \eta\right)\right] \\
\Psi_{\varepsilon, c, e}(x) & =\varepsilon(2 c)^{-1} A v_{|y-x| \leq \ell_{1}}\left[\eta_{x+c \varepsilon^{-1} e}-\eta_{x-c \varepsilon^{-1} e}\right] .
\end{aligned}
$$

Here we continue to follow the convention set up after (3.26) in step 3 of the proof of Theorem 3.3 that the generator $\mathcal{L}$ does not apply to the variable $\bar{\eta}_{x, \ell}$ because the error terms were proved to be negligible. We can rewrite

$$
\Phi_{x, e}^{\mathrm{g}}+\sum_{e^{\prime}} D_{e, e^{\prime}}\left(\bar{\eta}_{x, a \varepsilon^{-1}}\right) \Psi_{\varepsilon, c, e^{\prime}}=\Phi_{x, e}^{\mathbf{g}, s}+\Psi_{x, e}^{1, s}+\Psi_{x, e}^{2, s}+\Psi_{x, e}^{3}
$$

where

$$
\begin{aligned}
\Phi_{x, e}^{\mathbf{g}, s} & =A v_{|y-x| \leq \ell_{1}} \phi_{x, \varepsilon}^{g, y, s} \\
\phi_{x, e}^{g, y, s} & =\left[w_{y, y+e}+\mathcal{L} g_{e}\left(\bar{\eta}_{x, \ell}, \tau_{y} \eta\right)+\sum_{e^{\prime}} D_{e, e^{\prime}}\left(\bar{\eta}_{x, \ell}\right)\left(\nabla_{e^{\prime}} \eta_{y}-E^{\mu}\left[\nabla_{e^{\prime}} \eta_{y} \mid \mathcal{F}_{y, s}\right]\right)\right] \\
\Psi_{x, e}^{1, s} & =\sum_{e^{\prime}} D_{e, e^{\prime}}\left(\bar{\eta}_{x, a \varepsilon^{-1}}\right) A v_{|y-x| \leq \ell_{1}} E^{\mu}\left[\nabla_{e^{\prime}} \eta_{y} \mid \mathcal{F}_{y, s}\right] \\
\Psi_{x, e}^{2, s} & =\sum_{e^{\prime}}\left[D_{e, e^{\prime}}\left(\bar{\eta}_{x, a \epsilon^{-1}}\right)-D_{e, e^{\prime}}\left(\bar{\eta}_{x, \ell}\right)\right]\left[A v_{|y-x| \leq \ell_{1}}\left(\nabla_{e^{\prime}} \eta_{y}-E^{\mu}\left[\nabla_{e^{\prime}} \eta_{y} \mid \mathcal{F}_{y, s}\right]\right)\right] \\
\Psi_{x, e}^{3} & =\sum_{e^{\prime}} D_{e, e^{\prime}}\left(\bar{\eta}_{x, a \varepsilon^{-1}}\right)\left[A v_{|y-x| \leq \ell_{1}}\left[\Psi_{\varepsilon, c, e^{\prime}}(y)-\nabla_{e^{\prime}} \eta_{y}\right]\right]
\end{aligned}
$$

Hence we can establish the eigenvalue estimate of (3.28) by showing

$$
\limsup _{s, a, c, \ell, \varepsilon} \Omega_{0} \leq C(\gamma)\|J\|_{\infty}^{2} \sup _{\sum_{e} \alpha_{e}^{2}=1} \sup _{\beta} V\left(\sum_{e} \alpha_{e} \phi_{e}(\beta, \mathbf{g}), \beta\right)
$$

and for $i=1,2,3$,

$$
\limsup _{s, a, c, \ell, \varepsilon} \Omega_{i}=0
$$

where

$$
\begin{aligned}
& \Omega_{0}=\sup \operatorname{spec}\left[\epsilon^{-1}(\mathbf{J}, \Phi \mathbf{g}, s)-(\gamma / 4) \epsilon^{-2} A v_{b}\left(-\mathcal{L}_{b}\right)\right] \\
& \Omega_{i}=\sup \operatorname{spec}\left[\epsilon^{-1}\left(\mathbf{J}, \Psi^{i, s}\right)-(\gamma / 4) \epsilon^{-2} A v_{b}\left(-\mathcal{L}_{b}\right)\right], i=1,2 \\
& \Omega_{3}=\sup \operatorname{spec}\left[\epsilon^{-1}\left(\mathbf{J}, \Psi^{3}\right)-(\gamma / 4) \epsilon^{-2} A v_{b}\left(-\mathcal{L}_{b}\right)\right]
\end{aligned}
$$


and the inner product $($,$) is as defined in Theorem 3.9.$

We first consider $\Omega_{1}$. From (5.8), we have (7.3) holds for $i=1$. For $i=2$, we apply (5.7) to reduce the problem to showing

$\limsup _{s, \ell, a, \varepsilon} \sup \operatorname{spec}\left[C(s, \gamma, J) A v_{x}\left(D\left(\bar{\eta}_{x, a \epsilon^{-1}}\right)-D\left(\bar{\eta}_{x, \ell}\right)\right)^{2}-(\gamma / 8) \epsilon^{-2} A v_{b}\left(-\mathcal{L}_{b}\right)\right]=0$

which is the familiar form of two block estimate. From (6.3) we conclude (7.5). Note that we take $\ell \rightarrow \infty$ before taking the limit $s \rightarrow \infty$.

To deal with $\Omega_{3}$ we rewrite

$$
\left(J, \Psi^{3}\right)=\sum_{e^{\prime}} A v_{x}\left[G_{x, \varepsilon^{\prime}}(J, \eta) \nabla_{e^{\prime}} \eta_{x}\right]
$$

and use (7.6) to control $\lim \sup _{a, c, \ell, \varepsilon} \Omega_{3} . G$ is easily seen to satisfy the hypotheses needed to apply (7.7) . The problem reduces to one of showing

$$
\limsup _{a, c, \ell, \varepsilon} \sup \operatorname{spec}\left[A v_{x} G_{x, \varepsilon}^{2}-(\gamma / 8) \varepsilon^{-2} A v_{b}\left(-\mathcal{L}_{b}\right] \leq 0\right.
$$

which is easily seen to follow from the two block estimate.

Finally we have to bound $\Omega_{0}$. Note that $E^{\mu}\left[\phi_{x, e}^{\mathbf{g}, y, s} \mid \mathcal{F}_{y, s}\right]=0$ if $|y-x| \leq \ell_{1}$ and hence from Lemma 5.2 we have

$$
\Omega_{0} \leq C \sup s p e c\left[A v_{x} V_{\ell}\left(\sum_{e>0} J_{e}(\varepsilon x) \phi_{x, e}^{\mathbf{g}, x, s}, \bar{\eta}_{x, \ell}\right)-(\gamma / 8) \epsilon^{-2} A v_{b}\left(-\mathcal{L}_{b}\right)\right]+o(1)
$$

with $\lim _{s, \ell, a, \varepsilon} o(1)=0$. Note we have kept some Dirichlet form. For $k \geq \ell$ let

$$
U_{x, k}=E^{\mu_{\varepsilon}}\left[V_{\ell}\left(\sum_{e>0} J_{e}(\varepsilon x) \phi_{x, e}^{\mathbf{g}, x, s}, \bar{\eta}_{x, \ell}\right) \mid \mathcal{F}_{x, k}\right]
$$

and we can bound $\Omega_{0} \leq \Omega_{4}+\Omega_{5}+o(1)$ with

$$
\begin{aligned}
& \Omega_{4}=C \sup s p e c\left[A v_{x}\left\{V_{\ell}\left(\sum_{e>0} J_{e}(\varepsilon x) \phi_{x, e}^{\mathbf{g}, x, s}, \bar{\eta}_{x, \ell}\right)-U_{x, k}\right\}-(\gamma / 8) \epsilon^{-2} A v_{b}\left(-\mathcal{L}_{b}\right)\right] \\
& \Omega_{5}=\sup _{x, \mathcal{F}_{x, k}} U_{x, k}
\end{aligned}
$$

Using Lemma 5.2 and noting that the factor $\epsilon^{-1}$ in front of $h$ in (5.2) is not present in this calculation, we have

$$
\lim _{\varepsilon \rightarrow 0} \Omega_{4}=0 .
$$

It remains to bound $\Omega_{4}$. By the translational invariance,

$$
\Omega_{5} \leq \sup _{\omega, \rho} E^{\mu_{\Lambda_{2 k+1}, \omega, \rho}}\left[V_{\ell}\left(\sum_{e>0} J_{e}(\varepsilon x) \phi_{0, e}^{\mathbf{g}, 0, s}, \bar{\eta}_{\ell}\right) \mid \mathcal{F}_{k}\right]
$$


where $\mu_{\Lambda_{2 k+1}, \omega, \rho}$ denote the canonical Gibbs measure on $\Lambda_{2 k+1}$ with boundary condition $\omega$ and density $\rho$. For every $\ell$ fixed, we can take the limit $k \rightarrow \infty$ and any limiting point of the right hand side of (7.8) is given by

$$
E^{\mu_{\beta}}\left[V_{\ell}\left(\sum_{e} J(\varepsilon x) \phi_{0, e}^{\mathbf{g}, 0, s}, \bar{\eta}_{\ell}\right)\right]
$$

where $\mu_{\beta}$ is the unique Gibbs state with density $\beta$ given by the limit of $\bar{\eta}_{\ell}$. Note that from our mixing assumption, there is only one Gibbs state for a given density. From the law of large number of Gibbs state, we can replace the density $\bar{\eta}_{0, \ell}$ in the definition of $\phi_{0, e}^{\mathbf{g}, 0, s}$ in (7.9) by the total density $\beta$. Recall $\phi_{e}^{\mathbf{g}}(\beta)$ is given by (3.13) and by definition of $\phi_{0, e}^{\mathbf{g}, 0, s}$ we have

$$
\phi_{0, e}^{\mathbf{g}, 0, s}=\phi_{e}^{\mathbf{g}}(\beta)-E^{\mu_{\varepsilon}}\left[\phi_{e}^{\mathbf{g}}(\beta) \mid \mathcal{F}_{s}\right]
$$

for $s$ large enough; where the density $\bar{\eta}_{0, \ell}$ in $\phi_{0, e}^{\mathbf{g}, 0, s}$ is replaced by $\beta$. We can now take the limit $\ell \rightarrow \infty$ and then $s \rightarrow \infty$ to have

$$
\limsup _{\ell \rightarrow \infty} E^{\mu_{\beta}}\left[V_{\ell}\left(\sum_{e>0} J_{e}(\varepsilon x) \phi_{0, e}^{\mathbf{g}, 0, s}, \bar{\eta}_{\ell}\right)\right] \leq V\left(\sum_{e>0} J_{e}(\varepsilon x) \phi_{e}^{\mathbf{g}}(\beta), \beta\right)
$$

where we have used the definition (3.12) of $V$ This proves Theorem 3.9.

8. Computation of variance. Let us recall the definition of $\mathcal{G}$

$$
\mathcal{G}=\left\{h: E^{\mu}\left[h \mid \mathcal{F}_{s}\right]=0 \text { for some } s\right\}
$$

We would like to define the variance

$$
V(h, h ; \beta)=\lim _{\ell \rightarrow \infty} E^{\mu_{\beta}}\left[<h^{(\ell)}, \mathcal{L}_{(\ell)}^{-1} h^{(\ell)}>_{\ell, \omega, y}\right]
$$

as a uniform limit in $\beta$. Since we cannot show the existence of this limit at this time we define the upper and lower limits

$$
V^{*}(h, \beta)=\limsup _{\substack{\ell \rightarrow \infty \\ \beta^{\prime} \rightarrow \beta}} E^{\mu_{\beta^{\prime}}}\left[<h^{(\ell)}, \mathcal{L}_{(\ell)}^{-1} h^{(\ell)}>_{\ell, \omega, y}\right]
$$

and

$$
V_{*}(h, \beta)=\liminf _{\substack{\ell \rightarrow \infty \\ \beta^{\prime} \rightarrow \beta}} E^{\mu_{\beta^{\prime}}}\left[<h^{(\ell)}, \mathcal{L}_{(\ell)}^{-1} h^{(\ell)}>_{\ell, \omega, y}\right]
$$

Here

$$
h^{(\ell)}=\frac{1}{\left(2 \ell_{1}+1\right)^{d}} \sum_{x \in \Lambda_{l_{1}}} \tau_{x} h
$$

with $\ell_{1}=\ell-\sqrt{\ell}$ and

$$
\mathcal{L}_{(\ell)}=\frac{1}{(2 \ell+1)^{d}} \sum_{b \in \Lambda_{\ell}} \mathcal{L}_{b}
$$


For any $h \in \mathcal{G}$, for sufficiently large $\ell, E^{\mu_{\ell, \omega, y}}\left[h^{(\ell)}\right]=0$. The following calculation is a typical way to estimate (8.2). From $E^{\mu_{s, \omega, y}}[h]=0$, we conclude that

$$
h=\mathcal{L}_{(s)} u_{s}
$$

for some $u_{s}$. If we denote by $C_{s}^{-1}$ a lower bound on the spectral gap for a box of size $s$, for any function $v$ of the configurations in the box $\Lambda_{\ell}$

$$
\begin{aligned}
\left|E\left[h_{(\ell)} v\right]\right| & \leq \frac{1}{\left(2 \ell_{1}+1\right)^{d}} \sum_{x \in \Lambda_{\ell_{1}}}\left|E\left[\tau_{x} h v\right]\right| \\
& \leq \frac{\sqrt{C_{s}}}{\left(2 \ell_{1}+1\right)^{d}} \sum_{x \in \Lambda_{\ell_{1}}} \sqrt{E\left[\left[\tau_{x} h\right]^{2}\right]} \sqrt{\sum_{b \in \Lambda_{x}, s} D_{b}(v)} \\
& \leq \sqrt{C_{s}}(2 s+1)^{\frac{d}{2}} \frac{(2 \ell+1)^{\frac{d}{2}}}{\left(2 \ell_{1}+1\right)^{\frac{d}{2}}}\|h\|_{2, \ell}<v, \mathcal{L}_{(\ell)} v>^{\frac{1}{2}}
\end{aligned}
$$

providing us the bound

$$
<h^{(\ell)}, \mathcal{L}_{(\ell)}^{-1} h^{(\ell)}>_{\omega, y} \leq C_{s}\left(\frac{\ell}{\ell_{1}}\right)^{d}(2 s+1)^{d}\|h\|_{2, \ell}^{2}
$$

where $E$ denotes expectation with respect to any canonical Gibbs measure $\mu_{\ell, \omega, y}$ and

$$
\|h\|_{2, \ell}^{2}=\frac{1}{\left(2 \ell_{1}+1\right)^{2}} E\left[\sum_{x \in \Lambda_{\ell_{1}}}\left|\tau_{x} h\right|^{2}\right]
$$

Taking expectations with respect to $\mu^{\beta^{\prime}}$, letting $\ell \rightarrow \infty$ and $\beta^{\prime} \rightarrow \beta$ we have

$$
V^{*}(h, \beta) \leq C_{s}(2 s+1)^{d}\|h\|_{2, \beta}^{2}
$$

Because we defined $V^{*}(h, \beta)$ as the limsup, and did not show the existence of the limit, it is not quite clear that $V^{*}(h, \beta)$ is a quadratic form in $h$, and consequently it is not possible to generate the inner product from it by the standard polarization formula:

$$
V^{*}(h ; g, \beta)=\frac{1}{4}\left[V^{*}(g+h, \beta)-V^{*}(g-h, \beta)\right]
$$

Because $V_{\ell}(h ; \omega, y)$ is actually a quadratic form, it is possible to polarize it and try to calculate directly the quantity

$$
V(g, h ; \beta)=\lim _{\substack{\ell \rightarrow \infty \\ \beta^{\prime} \rightarrow \beta}} E^{\mu_{\beta^{\prime}}}\left\{V_{\ell}(g, h ; \omega, y)\right\}
$$

We will show that the limit exists and calculate it explicitly for a large class of local functions $g$ and $h$. Let us define the shifts $\tau_{x}$ for $x \in \mathbb{Z}^{d}$ by

$$
\left(\tau_{x} \eta\right)_{y}=\eta_{y-x}, \quad \tau_{x} b=b-x, \quad\left(\tau_{x} g\right)(\eta)=g\left(\tau_{x} \eta\right)
$$

on configurations $\eta$, bonds $b$ and functions $g$. We have the basic exchange operator $S_{b}$ defined by $S_{b} \eta=\eta^{b}=\eta^{x, y}$ for a bond $b=(x, y)$ in (2.10). The operator $T_{b}$ was 
already defined as $\left(T_{b} g\right)(\eta)=g\left(S_{b} \eta\right)-g(\eta)$. The shift operators $\tau_{x}$ and the exchange operators $S_{b}$ satisfy the commutation relations

$$
\tau_{x} S_{b} \eta=S_{b+x} \tau_{x} \eta, \quad\left(S_{b} \tau_{x} g\right)(\eta)=\left(\tau_{x} S_{b+x} g\right)(\eta)
$$

leading to the identities

$$
\left(T_{b} \tau_{x} g\right)(\eta)=\left(\tau_{x} T_{b+x} g\right)(\eta)=\left(\tau_{x} T_{\tau_{-x} b} g\right)(\eta)
$$

Suppose $g \in \mathcal{G}$, i.e it is a local functions such that $E^{\mu}\left\{g \mid \mathcal{F}_{s}\right\}=0$ for some $s$, then for any other local function $h, E^{\mu}\left[g \tau_{x} h\right]=0$ provided $\tau_{x} h$ does not depend on any of the variables $\left\{\eta_{z}: z \in \Lambda_{s}\right\}$. In particular for any $h$ which is a local function and $g \in \mathcal{G}$ the series

$$
\sum_{x} E^{\mu}\left[g \tau_{x} h\right]=<g, h>_{0}
$$

is well defined as a finite sum. If it happens that $h \in \mathcal{G}$ as well, then

$$
<g, h>_{0}(\beta)=\sum_{x} E^{\mu_{\beta}}\left[g \tau_{x} h\right]=\sum_{x} E^{\mu_{\beta}}\left[g \tau_{x} h\right]=<h, g>_{0}(\beta)
$$

is a continuous function of $\beta$ and

$$
<g, g>_{0}(\beta)=\lim _{\ell \rightarrow \infty} \frac{1}{(2 \ell+1)^{d}} E^{\mu_{\beta}}\left[\left(\sum_{x \in \Lambda_{\ell}} \tau_{x} g\right)^{2}\right] \geq 0
$$

so that for each $\beta,<,>_{0}$ is a positive semidefinite inner product. We will also need the quantities

$$
t_{e}(g, \beta)=\sum_{x}(e, x) E^{\mu_{\beta}}\left[\eta_{x} g\right]
$$

which are again only finite sums for $g \in \mathcal{G}$. Let $u$ be a local function with $\mathcal{L} u=g$. Clearly $g \in \mathcal{G}$ and $\mathcal{L} \tau_{x} u=\tau_{x} g$ for all $x \in \mathbb{Z}^{d}$. In particular

$$
\mathcal{L}_{\ell}\left(\sum_{x \in \Lambda_{\ell_{1}}} \tau_{x} u\right)=\mathcal{L}\left(\sum_{x \in \Lambda_{\ell_{1}}} \tau_{x} u\right)=\sum_{x \in \Lambda_{\ell_{1}}} \tau_{x} g
$$

If $v$ is now another local function with $\mathcal{L} v=h$, a direct calculation yields

$$
V_{\ell}(g, h ; \omega, y)=-\frac{1}{\left(2 \ell_{1}+1\right)^{d}} E^{\mu_{\ell, \omega, y}}\left\{\sum_{x, y \in \Lambda_{\ell_{1}}} \tau_{y} g \tau_{x} v\right\}
$$

and one can easily show that the limit

$$
V(g, h ; \beta)=\lim _{\substack{\ell \rightarrow \infty \\ \beta^{\prime} \rightarrow \beta}} E^{\mu_{\beta^{\prime}}}\left\{V_{\ell}(g, h ; \omega, y)\right\}=-\sum_{x \in \mathbb{Z}^{d}} E^{\mu_{\beta}}\left\{g \tau_{x} v\right\}=-<g, v>_{0}(\beta)
$$

exists.

The following calculation is easy to carry out. For each local function $u$ let us define the formal sum

$$
\mathbf{u}=\sum_{x} \tau_{x} u
$$


Although $\mathbf{u}$ is not well defined,

$$
\xi_{b}=T_{b} \mathbf{u}
$$

is well defined for every $b$ and

$$
\tau_{x} \xi_{b}=\xi_{b-x}
$$

for every $x$ and $b$. If we denote $T_{0, e}$ by $T_{e}$, and $\xi_{0, e}$ by $\xi_{e}$, we have

$$
<g, g>_{0}(\beta)=-<\mathcal{L} u, u>_{0}(\beta)=\frac{1}{2} E^{\mu_{\beta}}\left[\sum_{e} c_{e}(\eta)\left[\xi_{e}\right]^{2}\right]
$$

and by polarization

$$
<g, h>_{0}(\beta)=-<\mathcal{L} u, v>_{0}(\beta)=\frac{1}{2} E^{\mu_{\beta}}\left[\sum_{e} c_{e}(\eta) \xi_{e} \zeta_{e}\right]
$$

where

$$
\zeta_{e}=T_{e} \mathbf{v}=T_{e} \sum_{x} \tau_{x} v
$$

We next take $g=w_{e}=w_{0, e}$. From the definition of the current $w_{b}$ in (3.4) it is easy to establish the following identity, which is essentially an integration by parts formula, for any $\mu$ which is either $\mu_{\beta}$ for some $\beta$ or any canonical Gibbs measure $\mu_{\Lambda}^{\omega, y}$ on a box $\Lambda$ that contains the bond $(x, x+e)$.

$$
E^{\mu}\left[w_{x, x+e} u\right]=-E^{\mu}\left[\left(\eta_{x+e}-\eta_{x}\right) c_{x, x+e}(\eta)\left(T_{x, x+e} u\right)(\eta)\right]
$$

If we take any set $B \subset \Lambda$, it follows that

$$
\left|E^{\mu_{\Lambda}^{\omega, y}}\left[\left(\sum_{b \in B} w_{b}\right) u\right]\right|^{2} \leq E^{\mu_{\Lambda}^{\omega, y}}\left[\sum_{b \in B} c_{b}(\eta)\left(\nabla_{b} \eta\right)^{2}\right] E^{\mu_{\Lambda}^{\omega, y}}\left[\sum_{b \in B} c_{b}\left[\left(T_{b} u\right)(\eta)\right]^{2}\right]
$$

In particular

$$
<\sum_{b \in B} w_{b} ; \mathcal{L}_{\ell}^{-1} \sum_{b \in B} w_{b}>_{\omega, y} \leq C|B|
$$

Therefore in the definition of $V\left(w_{e}, w_{e^{\prime}} ; \beta\right)$ or can take

$$
V_{\ell}\left(w_{e}, w_{e^{\prime}} ; \omega, y\right)=<A v_{\Lambda_{\ell}} w_{e},\left[A v_{\Lambda_{\ell}} \mathcal{L}_{b}\right]^{-1} A v_{\Lambda_{\ell}} w_{e}>_{\omega, y}
$$

and because

$$
\mathcal{L}_{\Lambda_{\ell}} \sum_{x \in \Lambda_{\ell}}<x, e>\eta_{x}=\sum_{x \in \Lambda_{\ell}} w_{x, x+e}
$$

it is easy to calculate that

$$
V\left(w_{e}, w_{e^{\prime}} ; \beta\right)=\frac{\delta_{e, e^{\prime}}}{2} E^{\mu_{\beta}}\left[c_{e}(\eta)\left(\nabla_{e} \eta\right)^{2}\right]
$$

and for any local $u$

$$
V\left(w_{e}, \mathcal{L} u ; \beta\right)=\frac{1}{2} E^{\mu_{\beta}}\left[c_{e}(\eta)\left(T_{e} \sum_{x} \tau_{x} u\right) \nabla_{e} \eta\right],
$$


and in fact for any $g \in \mathcal{G}$

$$
V\left(w_{e}, g ; \beta\right)=t_{e}(g, \beta) .
$$

We collect these results as a lemma.

Lemma 8.1 If we define the class $\mathcal{G}_{0} \subset \mathcal{G}$ of functions as those which are of the form $g=\mathcal{L} u+\sum_{e} \alpha_{e} w_{e}$ for some local $u$, then $V(g, h ; \beta)$, given by (8.6) -(8.9), is a well defined, possibly degenerate inner product on $\mathcal{G}_{0}$. In addition $V(g, h ; \beta)$ exists as a limit in the sense of (8.5) so long as $g, h \in \mathcal{G}$ and either $g$ or $h$ is in $\mathcal{G}_{0}$.

Our next goal is to show that $\mathcal{G}$ is not all that much larger than $\mathcal{G}_{0}$. We want to show that, for any $\beta, \mathcal{G}$ is contained in the completion $\overline{\mathcal{G}}_{0}$ of $\mathcal{G}_{0}$ relative to the inner product $V(\cdot, \cdot ; \beta)$. More precisely with $V(h, h ; \beta)$ defined as the limsup for $h \in \mathcal{G}$ we want to show that

$$
\inf _{g \in \mathcal{G}_{0}} V^{*}(h-g, h-g ; \beta)=0
$$

Notice that although $V^{*}$, defined by(8.2), may not be a quadratic form it is still true that for $g \in \mathcal{G}_{0}$ and $h \in \mathcal{G}$

$$
V^{*}(h-g, h-g ; \beta)=V^{*}(h, h ; \beta)-2 V(h, g ; \beta)+V(g, g ; \beta)
$$

so that (8.10) reduces to proving the variational formula

$$
V^{*}(h, h ; \beta)=\sup _{g \in \mathcal{G}_{0}}\{2 V(h, g ; \beta)-V(g, g ; \beta)\}
$$

The variational formula (8.11) also proves that for $V_{*}$ defined by (8.3)

$$
\begin{aligned}
V_{*}(h, h ; \beta) & =V_{*}(h-g, h-g ; \beta)+2 V(h-g, g ; \beta)+V(g, g ; \beta) \\
& \geq \sup _{g \in \mathcal{G}_{0}}[2 V(h, g ; \beta)-V(g, g ; \beta)] \\
& =V^{*}(h, h ; \beta)
\end{aligned}
$$

establishing the equality $V^{*}=V_{*}$ and therefore $V_{*}=V^{*}=V(h, h ; \beta)$ exists as a limit and defines a quadratic form for all $h \in \mathcal{G}$. Let us remark that from the definition $V^{*}$ is upper semicontinuous in $\beta$, while $V_{*}$ is lower semicontinuous. In particular the equality implies the continuity in $\beta$. We now state these results as a theorem. Since $\beta$ will be fixed from now on, we will drop all references to it.

THEOREM 8.2 For $h \in \mathcal{G}$ the variance $V(h, h)$ satisfies the variational formula

$$
\begin{aligned}
\frac{1}{2} V(h, h)= & \sup _{\alpha_{e} \in R, u \in \mathcal{G}}\left[V\left(h, \sum_{e>0} \alpha_{e} w_{e}-\mathcal{L} u\right)-\frac{1}{2} V\left(\sum_{e>0} \alpha_{e} w_{e}-\mathcal{L} u\right)\right] \\
= & \sup _{\alpha_{e} \in R, u \in \mathcal{G}}\left\{\sum_{e>0} \alpha_{e} t_{e}(h)+\langle h, u\rangle_{0}\right. \\
& \left.\quad-1 / 4 \sum_{e>0} E^{\mu}\left[c_{e}(\eta)\left(\alpha_{e} \nabla_{e} \eta+T_{e} \sum_{x} \tau_{x} u\right)^{2}\right]\right\}
\end{aligned}
$$

The equivalence of the two formulae in (8.12) follows from Lemma 8.1. Hence we only have to prove the first identity in (8.12). This will be the main thrust of the rest of this paper. 
We now assume Theorem 8.2 and conclude the proof of Theorem 3.4. In general $\nabla_{e} \eta \notin \mathcal{G}$. However for any $e$ and $s, h_{e, s}=\nabla_{e} \eta-E^{\mu}\left[\nabla_{e} \eta \mid \mathcal{F}_{s}\right] \in \mathcal{G}$ and for $s_{1}<s_{2}$, by (8.4) and the estimate on the spectral gap

$$
V\left(h_{e, s_{1}}-h_{e, s_{2}}, h_{e, s_{1}}-h_{e, s_{2}}\right) \leq C s_{2}^{d+2} s_{1}^{-(d+2+\alpha)} .
$$

This is enough to prove that $h_{e, s}$ is a Cauchy sequence (uniformly with respect to $\beta$ ) in $\mathcal{G}$ and letting $s \rightarrow \infty, h=\lim _{s \rightarrow \infty} h_{e, s}$ gets represented in $\overline{\mathcal{G}}$. Using (8.6) and (8.9) we can explicitly calculate

$$
V\left(w_{e}, \nabla_{e^{\prime}} \eta\right)=t_{e}\left(\nabla_{e^{\prime}} \eta\right)=\delta_{e, e^{\prime}} \sum_{x}<\eta_{0} ; \eta_{x}>_{\mu}=\delta_{e, e^{\prime}} \chi
$$

and

$$
V\left(\mathcal{L} u, \nabla_{e} \eta\right)=0
$$

Proof of Theorem 3.4. Let

$$
\mathcal{G}^{(0)}=\left\{\sum_{e>0} \alpha_{e} \nabla_{e} \eta_{0}\right\}
$$

We claim that $\mathcal{G}^{(0)}+\mathcal{L} \mathcal{G}$ is also dense in $\overline{\mathcal{G}}$ where $\mathcal{L} \mathcal{G}$ denotes the linear space $\{\mathcal{L} u\}$ as $u$ varies over all local functions. From $(8.14), \mathcal{G}^{(0)} \perp \mathcal{L G}$. From (8.13) we see that the projection of the space $\mathcal{G}^{(w)}$ onto $\mathcal{G}^{(0)}$ has rank equal to the dimension of $\mathcal{G}^{(w)}$. Therefore the dimensions of $\mathcal{G}^{(0)}$ and $\mathcal{G}^{(w)}$ are both $d$. Since $\mathcal{G}^{(w)}+\mathcal{L} \mathcal{G}$ is dense in $\overline{\mathcal{G}}$, it follows that $\overline{\mathcal{G}}=\mathcal{G}^{(0)} \oplus \overline{\mathcal{L G}}$.

Let $\xi_{e}$ denote the orthogonal projection of $w_{e}$ onto the space $\overline{\mathcal{L G}}$. Hence there is a diffusion matrix $D$ such that

$$
w_{e}=\frac{1}{2} \sum_{e *>0} D_{e, e *} \nabla_{e *} \eta_{0}+\xi_{e}
$$

as an identity in $\overline{\mathcal{G}}$. Clearly, the projection of $\sum_{e} \gamma_{e} w_{e}$ is $\sum_{e} \gamma_{e} \xi_{e}$. By definition, $\xi_{e}$ can be approximated by elements of the form $\mathcal{L} g_{e}$ and thus $\sum_{e} \gamma_{e} \xi_{e}$ can be approximated by $\mathcal{L} \sum_{e} \gamma_{e} g_{e}$. Therefore, (3.14) holds for some diffusion matrix $D$. Our final task is to prove the characterization $(2.22)$.

Using the current notation, we can rewrite the diffusion coefficient (2.22) as

$$
\frac{1}{2} \gamma \cdot D \cdot \gamma=\chi^{-1} \inf _{\zeta \in \mathcal{L} \mathcal{G}} V(\gamma \cdot w-\zeta)
$$

The minimizer is simply the projection of $\gamma \cdot w$ onto the space $\overline{\mathcal{L G}}$, namely, $\sum_{e} \gamma_{e} \xi_{e}$. Taking inner product of $(8.16)$ with $w_{e^{\prime}}$, we have

$$
V\left(w_{e}, w_{e^{\prime}}\right)=\frac{1}{2} \sum_{e *} D_{e, e *} V\left(\nabla_{e *} \eta_{0}, w_{e^{\prime}}\right)+V\left(\xi_{e}, w_{e^{\prime}}\right)
$$

We can compute $V\left(\nabla_{e *} \eta_{0}, w_{e^{\prime}}\right)$ by (8.13) and thus

$$
V\left(w_{e}, w_{e^{\prime}}\right)=\frac{1}{2} D_{e, e^{\prime}} \chi+V\left(\xi_{e}, w_{e^{\prime}}\right)
$$


Hence for any vector $\gamma$ we have

$$
\frac{1}{2} \gamma \cdot D \cdot \gamma=\chi^{-1}[V(\gamma \cdot w)-V(\gamma \cdot \xi, \gamma \cdot w)]
$$

Since $\gamma \cdot \xi$ is the projection of $\gamma \cdot w$ onto the space $\overline{\mathcal{L G}}$, we have

$$
V(\gamma \cdot \xi, \gamma \cdot \xi-\gamma \cdot w)=0
$$

Therefore we can rewrite (8.18) as

$$
\frac{1}{2} \gamma \cdot D \cdot \gamma=\chi^{-1}[V(\gamma \cdot w)-2 V(\gamma \cdot \xi, \gamma \cdot w)+V(\gamma \cdot \xi)]=V(\gamma \cdot w-\gamma \cdot \xi)
$$

establishing the equivalence of (8.19) and (8.17). This proves Theorem 3.4 assuming Theorem 8.2.

We now prove that the diffusion matrix is diagonal if the model is isotropic, i.e., the Hamiltonian and the jump rate $A$ in (2.14) are isotropic.

LEMMA 8.3 Under the previous assumption, the diffusion coefficient is diagonal

Proof. Fix two coordinate direction $e$ and $e^{\prime}$. Taking inner product of (8.16) with $\nabla_{e^{\prime}} \eta_{0}$, we have

$$
V\left(w_{e}, \nabla_{e^{\prime}} \eta_{0}\right)=\frac{1}{2} \sum_{e *} D_{e, e *} V\left(\nabla_{e *} \eta_{0}, \nabla_{e^{\prime}} \eta_{0}\right)
$$

We can compute $V\left(\nabla_{e *} \eta_{0}, w_{e^{\prime}}\right)$ by (8.13). Hence we only have to prove that $V\left(\nabla_{e *} \eta_{0}\right.$, $\left.\nabla_{e^{\prime}} \eta_{0}\right)$ is diagonal. Denote by $\theta: \mathbb{Z} \rightarrow \mathbb{Z}$ the reflection with respect to the origin along the $e^{\prime}$ direction. We may extend $\theta$ to the space of configurations in the natural way: $(\theta \eta)(x)=\eta(\theta(x))$ and to the space of continuous functions: $(\theta f)(\eta)=f(\theta \eta)$. Clearly, $V(f, g)=V(\theta f, \theta g)$ since our model is isotropic. Notice that

$$
\theta\left(\nabla_{e^{\prime}} \eta(0)\right)=-\tau_{-e^{\prime}} \nabla_{e^{\prime}} \eta(0) ; \quad \theta\left(\nabla_{e} \eta(0)\right)=\nabla_{e} \eta(0), \quad \text { if } e \neq e^{\prime}
$$

Hence $V\left(\nabla_{e} \eta_{0}, \nabla_{e^{\prime}} \eta_{0}\right)=-V\left(\nabla_{e} \eta_{0}, \nabla_{e^{\prime}} \eta_{0}\right)=0$. This proves the Lemma.

Return to the proof of Theorem 8.2. We shall follow the approach of [V1] and [Q1]. We first need the notion of "closed forms". This is some what parallel to the usual notion of closed forms in differential calculus. Our goal is to characterize the 1-form arising from

$$
\omega_{b}=T_{b} g
$$

for some function $g$. Clearly, there are compatibility conditions for $\omega=\left\{\omega_{b}\right\}$. One can check easily that the following conditions hold:

$$
T_{b} \omega_{q}=T_{q} \omega_{b}, \text { if } q \cap b=\phi .
$$

Unlike in differential calculus, (8.21) fails if $q \cap b \neq \phi$ because the operators are not local. Hence (8.21) is not enough to characterize the forms arising in this manner. We therefore use instead the definition: $\omega=\left\{\omega_{b}\right\}$ is "closed" provided for any sequence of nearest neighbor bonds $\left\{b_{1}, b_{2}, \ldots, b_{n}\right\}$ such that $S_{b_{n}} S_{b_{n-1}} \ldots S_{b_{2}} S_{b_{1}}=I$ one has

$$
\sum_{i=1}^{n} \omega_{b_{i}}\left(S_{b_{i-1}} S_{b_{i-2}} \ldots S_{b_{1}} \eta\right)=0
$$


where $S_{b} \eta=\eta^{b}$. Let $P_{i} \eta$ denotes the new configuration obtained by applying the permutation $P_{i}=S_{b_{i}} S_{b_{i-1}} \ldots S_{b_{1}}$ to $\eta$. One can check easily that if $\omega$ is given by (8.20) then (8.22) holds. To prove this, we substitute $\omega_{b}=T_{b} g$ into the equation and we only have to check

$$
\sum_{i=1}^{n} T_{b_{i}} g\left(P_{i-1} \eta\right)=0
$$

By definition,

$$
T_{b_{i}} g\left(P_{i-1} \eta\right)=g\left(S_{b_{i}} P_{i-1} \eta\right)-g\left(P_{i-1} \eta\right)=g\left(P_{i} \eta\right)-g\left(P_{i-1} \eta\right)
$$

We see that (8.23) is a telescoping sum vanishes because $P_{n}=P_{0}=I$. Conversely, if (8.22) holds, we can reconstruct the function $g$ as long as we are in a finite cube $\Lambda$ with a fixed total number of particles. This can be achieved in a standard way.

For any two configuration $\eta$ and $\zeta$ with the same total number of particles, i.e., $|\eta|=|\zeta|$, we can construct a sequence $S_{b_{i}}, i=1, \cdots, n$ so that $\zeta=S_{b_{n}} \cdots S_{b_{1}} \eta$. Starting from a configuration $\eta$, we can define the value of the function $g$ at any configuration $\zeta$ with $|\eta|=|\zeta|$ by

$$
g(\zeta)=\sum_{i=1}^{n} \omega_{b_{i}}\left(S_{b_{i-1}} S_{b_{i-2}} \ldots S_{b_{1}} \eta\right)
$$

The condition (8.22) is exactly the compatibility condition that two different ways to reach $\zeta$ will yield the same value for $g(\zeta)$. Note that configurations with different total number of particles are completely unrelated. Hence in general we can require that

$$
E^{\mu}\left[g \mid \mathcal{F}_{L}\right]=0
$$

if the cube $\Lambda$ is of width $2 L+1$; here $\mathcal{F}_{L}$ is the $\sigma$-algebra defined in (3.8). This procedure can be performed in a finite cube and thus the function $g$ always exists locally, i.e., if we condition on the configuration outside a cube $\Lambda$, the condition (8.22) implies the existence of a function $g$ such that $S_{b} g=\omega_{b}$ for all bonds $b$ inside $\Lambda$. Therefore, (8.22) implies (8.21). In addition to the existence, we have the following bound

$$
E^{\mu}\left[g^{2}\right] \leq C L^{2} \sum_{b \in \Lambda_{L}} E^{\mu}\left[\omega_{b}^{2}\right]
$$

For the whole lattice $\mathbb{Z}^{d}$, it is more subtle and, indeed, the reconstruction is not necessarily valid. We shall discuss this in details in the next section. For now, we define the space of (translationally covariant) closed forms, $\mathcal{G}_{C}$, by

$$
\mathcal{G}_{C}=\left\{\omega=\left\{\omega_{b}\right\}: \omega \text { is translationally covariant and is closed }\right\}
$$

where $\omega$ is said to be translationally covariant if it satisfies

$$
\tau_{x} \omega_{b}(\eta)=\omega_{b}\left(\tau_{x} \eta\right)=\omega_{\tau_{x} b}(\eta)=\omega_{b-x}(\eta)
$$

The existence of translationally covariant closed 1-forms is not entirely obvious. The idea is that, if we start with a local function $u$ and define the formal sum

$$
\mathbf{u}=\sum_{x \in \mathbb{Z}^{d}} \tau_{x} u
$$


as we saw before, although $\mathbf{u}$ does not really exist as a translation invariant function, its formal "gradients"

$$
\omega_{b}=T_{b} \mathbf{u}
$$

are well defined local functions and constitute a translationally invariant closed 1-form. These are to be thought of as exact forms. On the other hand for each $e$ the form

$$
\omega_{x, x+e^{\prime}}^{e}(\eta)=\delta_{e, e^{\prime}}\left[\eta_{x+e^{\prime}}-\eta_{x}\right]
$$

is a "closed" 1-form that is not of the exact type. The following Lemma is the first step in proving Theorem 8.2 and provides a variational formula for the variance in terms of "closed forms". The proof will be completed in the next section where we will prove that $\overline{\mathcal{G}}_{0}=\mathcal{G}_{C}$ in a suitable sense. .

Lemma 8.4 For $h \in \mathcal{G}$ the variance $V^{*}(h, h)$ satisfies the variational principle

$$
\frac{1}{2} V^{*}(h, h) \leq \sup _{\xi \in \mathcal{G}_{C}} E^{\mu}\left[\sum_{e>0} c_{e} \Phi_{e}^{h}(\eta) \xi_{e}\right]-\frac{1}{4} E^{\mu}\left[\sum_{e>0} c_{e}\left(\xi_{e}\right)^{2}\right]
$$

where, for each e, $\Phi_{e}^{h}(\cdot)$ is a local function depending on $h$ to be defined in (8.30) and (8.31). In addition there is also the counterpart

$$
\frac{1}{2} V_{*}(h, h) \geq \sup _{\xi \in \mathcal{G}_{0}} E^{\mu}\left[\sum_{e>0} c_{e} \Phi_{e}^{h}(\eta) \xi_{e}\right]-\frac{1}{4} E^{\mu}\left[\sum_{e>0} c_{e}\left(\xi_{e}\right)^{2}\right]
$$

with the same functions $\Phi_{e}^{h}(\cdot)$.

Proof. First we prove (8.29), which is the easy part. Clearly

$$
\frac{1}{2} V_{\ell}(h, h ; \omega, y)=\sup _{v}\left\{E^{\mu_{\ell, \omega} y}\left[\left[A v_{x \in \Lambda_{\ell_{1}}} \tau_{x} h\right] v\right]-\frac{1}{4} E^{\mu_{\ell, \omega} y}\left[A v_{b \in \Lambda_{\ell}}\left[c_{b}(\eta)\left(T_{b} v\right)^{2}\right]\right]\right\}
$$

By choosing

$$
v=\frac{1}{(2 \ell+1)^{d}}\left[\sum_{x \in \Lambda_{\ell}}<\alpha, x>\eta_{x}-\sum_{x \in \Lambda_{\ell_{1}}} \tau_{x} u\right]
$$

for some local $u$, taking expectations with respect $\mu_{\beta^{\prime}}$, letting $\ell \rightarrow \infty$ while $\beta^{\prime} \rightarrow \beta$, we get

$$
-\frac{1}{4} E^{\mu}\left[\sum_{e} c_{e}(\eta) \xi_{e}^{2}\right]
$$

for the second term, where

$$
\xi_{e}=\alpha_{e} \nabla_{e} \eta_{0}-T_{e} \sum_{x \in \mathbb{Z}^{d}} \tau_{x} u
$$

Since $h \in \mathcal{G}$ we can write

$$
h=\mathcal{L}_{s} H=\sum_{b \in \Lambda_{s}} \mathcal{L}_{b} H
$$

with $H$ measurable with respect to some $\mathcal{K}_{s+R}$ (see (3.9)), and calculate

$$
\begin{aligned}
& E^{\mu} E^{\mu_{\ell, \omega y}}\left[\left[A v_{x \in \Lambda_{\ell_{1}}} \tau_{x} h\right] v\right]=E^{\mu}\left[\left(A v_{x \in \Lambda_{\ell_{1}}} \tau_{x} \sum_{b \in \Lambda_{s}} \mathcal{L}_{b} H\right) v\right] \\
& =-\frac{1}{2} \sum_{b \in \Lambda_{s}} A v_{x \in \Lambda_{\ell_{1}}} E^{\mu}\left[\tau_{x}\left[c_{b}(\eta)\left(T_{b} H\right)\right]\left(T_{b-x} v\right)\right]
\end{aligned}
$$


It is now easily seen that the first term reduces to

$$
-\frac{1}{2} E^{\mu}\left[\sum_{e} c_{e}(\eta)\left(\sum_{y: \tau_{y} e \in \Lambda_{s}} \tau_{y} T_{\tau_{y}} H\right) \xi_{e}=E^{\mu}\left[\sum_{e} c_{e}(\eta) \Phi_{e}^{h}(\eta) \xi_{e}(\eta)\right]\right.
$$

with

$$
\Phi_{e}^{h}(\eta)=-\frac{1}{2} \sum_{y: \tau_{y} e \in \Lambda_{s}} \tau_{y} T_{\tau_{y} e} H
$$

thereby proving (8.32) . We now turn to the proof of (8.28).

Step1. Let

$$
V^{*}=\limsup _{\substack{\ell \rightarrow \infty \\ \beta^{\prime} \rightarrow \beta}} E^{\mu_{\beta^{\prime}}}\left[V_{\ell}(h, h ; \omega, y)\right]
$$

and suppose that $\ell \gg 1$ and $\beta^{\prime} \sim \beta$ have been chosen so that, for some small $\delta>0$, with $\mu^{\prime}=\mu_{\beta^{\prime}}$,

$$
E^{\mu^{\prime}}\left[V_{\ell}(h, h ; \omega, y)\right] \geq V^{*}-\delta .
$$

We have the usual variational principle

$$
\frac{1}{2} V_{\ell}(h, h ; \omega, y)=\sup _{u}\left\{E^{\mu_{\ell \omega y}}\left[u A v_{|x| \leq \ell_{1}} h\right]-\frac{1}{4} E^{\mu_{\ell \omega y}}\left[A v_{|b| \leq \ell} c_{b}(\eta)\left(T_{b} u\right)^{2}\right]\right\}
$$

that is valid for each $\omega$. Integration with respect to the infinite volume Gibbs measure $\mu^{\prime}$ yields

$$
\frac{1}{2} E^{\mu^{\prime}}\left[V_{\ell}(h, h ; \omega, y)\right]=\sup _{u}\left\{A v_{|x| \leq \ell_{1}} E^{\mu^{\prime}}\left[\left(\tau_{x} h\right) u\right]-\frac{1}{4} A v_{|b| \leq \ell} E^{\mu^{\prime}}\left[c_{e}(\eta)\left(T_{b} u\right)^{2}\right]\right\}
$$

Hence, there exists a function $u=u^{(\ell)}$ such that

$$
\frac{1}{2} E^{\mu^{\prime}}\left[V_{\ell}(h, h ; \omega, y)\right] \leq A v_{|x| \leq \ell_{1}} E^{\mu^{\prime}}\left[\left(\tau_{x} h\right) u\right]-\frac{1}{4} A v_{|b| \leq \ell} E^{\mu^{\prime}}\left[c_{e}(\eta)\left(T_{b} u\right)^{2}\right]+\delta,
$$

where $\ell_{1}=\ell-\sqrt{\ell}$. Moreover we can assume without loss of generality that $u$ is a function of the configurations on $\Lambda_{\ell+R}$ and satisfies

$$
A v_{|x| \leq \ell_{1}} E^{\mu^{\prime}}\left[\left(\tau_{x} h\right) u\right]-\frac{1}{4} A v_{|b| \leq \ell} E^{\mu^{\prime}}\left[c_{e}(\eta)\left(T_{b} u\right)^{2}\right] \geq 0
$$

Since $h$ is a local function, it is measurable w.r.t. $\mathcal{K}_{s}$ for some $s$ large enough. Because $h \in \mathcal{G}$ (3.7), as we saw earlier, $h=\mathcal{L}_{s} H$ for some $H$ that is $\mathcal{K}_{s+R}$ measurable. Therefore, there is a constant $C(h)$ such that, for any constant $\gamma>0$, and $x \in \Lambda_{\ell_{1}}$

$$
E^{\mu^{\prime}}\left[\left(\tau_{-x} h\right) u\right] \leq(4 \gamma)^{-1} \sum_{|b-x| \leq s} E^{\mu^{\prime}}\left[c_{b}(\eta)\left(T_{b} u\right)^{2}\right]+\frac{\gamma}{2} C(h)
$$

Let $\gamma=2(2 s+1)^{d}$. Summing (8.36) over $x$ with $|x| \leq \ell_{1}$ and using (8.35), we have the following energy bound that holds uniformly in $\ell$ :

$$
A v_{|b| \leq \ell} E^{\mu}\left[c_{b}(\eta)\left(T_{b} u\right)^{2}\right] \leq C_{s} C(h)
$$


Step2. For $h \in \mathcal{G}(3.7),\left\{\Phi_{e}^{h}(\cdot)\right\}$ were defined by (8.30) and (8.31). From

$$
E^{\mu^{\prime}}\left[u h \mid \mathcal{F}_{s}\right]=E^{\mu^{\prime}}\left[u \mathcal{L}_{s} H \mid \mathcal{F}_{s}\right]
$$

we deduce that

$$
E^{\mu^{\prime}}\left[u h \mid \mathcal{F}_{s}\right]=-\frac{1}{2} \sum_{b \in \Lambda_{s}} E^{\mu^{\prime}}\left[c_{b}(\eta)\left(T_{b} H\right)(\eta)\left(T_{b} u\right)(\eta) \mid \mathcal{F}_{s}\right]
$$

and

$$
\begin{aligned}
& A v_{|x| \leq \ell_{1}} E^{\mu^{\prime}}\left[\left(\tau_{x} h\right) u\right] \\
= & -\frac{1}{2}\left(2 \ell_{1}+1\right)^{-d} E^{\mu^{\prime}}\left[\sum_{|x| \leq \ell_{1}} \sum_{b+x \in \Lambda_{s}} c_{b}(\eta)\left(T_{b} \tau_{x} H\right)(\eta)\left(T_{b} u\right)(\eta)\right] \\
= & \left(2 \ell_{1}+1\right)^{-d} E^{\mu^{\prime}}\left[\sum_{b \in \Lambda_{\ell}} c_{b}(\eta) \tilde{\Phi}_{b}(\eta)\left(T_{b} u\right)(\eta)\right]
\end{aligned}
$$

where for $b=\tau_{y} e \in \Lambda_{\ell}$,

$$
\tilde{\Phi}_{b}(\eta)=\sum_{\substack{x \in \Lambda_{\ell_{1}} \\ b+x \in \Lambda_{s}}}\left(T_{b} \tau_{x} H\right)(\eta)=\tau_{y} \Phi_{e}^{h}(\eta)+\Omega_{b}(\eta)
$$

where $\Omega_{b}$ are boundary terms that satisfy $\Omega_{b}=0$ for $b \in \Lambda_{\ell-2 \sqrt{\ell}}$ and $\left|\Omega_{b}\right| \leq C(h)$ otherwise. Clearly, from Schwarz's inequality, and (8.37)

$$
\frac{1}{\left(2 \ell_{1}+1\right)^{d}} \sum_{\substack{b \in \Lambda_{\ell} \\ b \notin \Lambda_{\ell-2 \sqrt{\ell}}}} E^{\mu^{\prime}}\left[c_{b}(\eta) \Omega_{b}(\eta)\left(\tau_{x} h\right) T_{b} u\right] \leq C \ell^{-\frac{1}{2}}
$$

and we can therefore replace

$$
A v_{|x| \leq \ell_{1}} E^{\mu^{\prime}}\left[\left(\tau_{x} h\right) u\right]
$$

in (8.33) with

$$
A v_{|x| \leq \ell_{1}} E^{\mu^{\prime}}\left[\tau_{x}\left[c_{e}(\eta) \Phi_{e}^{h}(\eta)\right] T_{\tau_{x} e} u\right]
$$

so that

$$
\left.A v_{|x| \leq \ell_{1}} E^{\mu^{\prime}}\left[\tau_{x}\left[c_{e}(\eta) \Phi_{e}^{h}(\eta)\right] T_{\tau_{x} e} u\right]\right]-\frac{1}{4} A v_{|b| \leq \ell} E^{\mu^{\prime}}\left[c_{b}(\eta)\left(T_{b} u\right)^{2}\right] \geq V^{*}-3 \delta
$$

Step 3. Let us pick $k \gg 1$ and fix it. There must be a good box of size $k$, i.e with $\Lambda_{x, k+R} \subset \Lambda_{\ell_{1}}$ such that

$$
\left.A v_{y \in \Lambda_{x, k}} E^{\mu^{\prime}}\left[\tau_{y}\left[c_{e}(\eta) \Phi_{e}^{h}(\eta)\right] T_{\tau_{x} e} u\right]\right]-\frac{1}{4} A v_{|b| \in \Lambda_{x, k}} E^{\mu^{\prime}}\left[c_{b}(\eta)\left(T_{b} u\right)^{2}\right] \geq V^{*}-4 \delta
$$

If we now replace $u=u^{(\ell)}$ by $\bar{u}^{(\ell)}=E^{\mu^{\prime}}\left[u^{(\ell)} \mid \mathcal{K}_{x, s+R}\right]$ we still have

$$
\left.A v_{y \in \Lambda_{x, k}} E^{\mu^{\prime}}\left[\tau_{y}\left[c_{e}(\eta) \Phi_{e}^{h}(\eta)\right] T_{\tau_{x} e} \bar{u}^{(\ell)}\right]\right]-\frac{1}{4} A v_{|b| \in \Lambda_{x, k}} E^{\mu^{\prime}}\left[c_{b}(\eta)\left(T_{b} \bar{u}^{(\ell)}\right)^{2}\right] \geq V^{*}-4 \delta
$$


by taking conditional expectations first. It is easy to pass to the limit as $\ell \rightarrow \infty$ and $\mu^{\prime} \rightarrow \mu$, taking a subsequence if needed. We produce in the limit a function $u_{k}$ such that it is $\mathcal{K}_{k}$ measurable and satisfies

$$
A v_{y \in \Lambda_{k}} E^{\mu}\left[\sum_{e} \tau_{y}\left[c_{e}(\eta) \Phi_{e}^{h}(\eta)\right] T_{\tau_{x} e} u_{k}\right]-\frac{1}{4} A v_{|b| \in \Lambda_{k}} E^{\mu}\left[c_{b}(\eta)\left(T_{b} u_{k}\right)^{2}\right] \geq V^{*}-4 \delta
$$

or

$$
E^{\mu}\left[\sum_{e} c_{e}(\eta) \Phi_{e}^{h}(\eta) T_{e}\left[A v_{y \in \Lambda_{k}} \tau_{y} u_{k}\right]\right]-\frac{1}{4} A v_{|b| \in \Lambda_{k}} E^{\mu}\left[c_{b}(\eta)\left(T_{b} u_{k}\right)^{2}\right] \geq V^{*}-4 \delta
$$

If we define $\xi_{e}^{k}=T_{e} A v_{y \in \Lambda_{k}}\left[\tau_{y} u_{k}\right]$ then

$$
\begin{aligned}
& E^{\mu}\left[\sum_{e} c_{e}(\eta)\left(\xi_{e}^{k}\right)^{2}\right] \\
= & E^{\mu}\left[\sum_{e} c_{e}(\eta)\left[A v_{y \in \Lambda_{k}} T_{\tau_{y} e} u_{k}\right]^{2}\right] \leq E^{\mu}\left[\sum_{e} c_{e}(\eta)\left[A v_{y \in \Lambda_{k}}\left(T_{\tau_{y} e} u_{k}\right)^{2}\right]\right] \\
= & A v_{|b| \in \Lambda_{k}} E^{\mu}\left[c_{b}(\eta)\left(T_{b} u_{k}\right)^{2}\right]
\end{aligned}
$$

We conclude that

$$
E^{\mu}\left[\sum_{e} c_{e}(\eta) \Phi_{e}^{h}(\eta) \xi_{e}^{k}\right]-\frac{1}{4} E^{\mu}\left[\sum_{e} c_{e}(\eta)\left(\xi_{e}^{k}\right)^{2}\right] \geq V^{*}-4 \delta
$$

While $\xi_{e}^{k}$ is not a covariant gradient it is routine to show that any limit point of $\xi_{e}^{k}$ as $k \rightarrow \infty$ is one. This concludes the proof.

It remains to establish that $\mathcal{G}_{0}$ is dense in $\mathcal{G}$ in a suitable sense, and this will be taken up in the next section.

9. Structure of closed forms. Our goal is to prove that any translationally covariant closed form $\xi=\left\{\xi_{e}\right\}$ can be approximated in $L^{2}(\mu)$ by forms of the type $\left\{\alpha_{e} \nabla_{e} \eta+T_{e} \sum_{x} \tau_{x} g\right\}$ with suitable scalars $\left\{\alpha_{e}: e>0\right\}$.

More precisely, let

$$
\mathcal{G}_{E}=\left\{\xi: \xi_{b}=T_{b} \sum_{x \in \mathbb{Z}^{d}} \tau_{x} g, \mathrm{~g} \text { is a local function }\right\}
$$

be the space of translationally covariant exact forms and $\mathcal{G}^{(1)}$ be the $d$-dimensional space of translationally covariant closed forms given by

$$
\mathcal{G}^{(1)}=\left\{\xi^{\alpha}: \xi_{b}^{\alpha}=\sum_{e>0} \alpha_{e} \delta_{b, e} \nabla_{b} \eta \quad \text { for some } \quad \alpha_{e} \in \boldsymbol{R}\right\}
$$

where $\delta_{b, e}=1$ if the bond is in the same direction as $e$, and 0 otherwise. Formally one can think of

$$
\xi_{b}^{\alpha}=-T_{b} \sum_{x \in \mathbb{Z}^{d}}<\alpha, x>\eta_{x}
$$


Clearly, these two spaces are subspaces of $\mathcal{G}_{C}$. Define the $L^{2}$ norm of a closed form by

$$
\|\xi\|_{2}^{2}=E^{\mu}\left[\sum_{e>0} \xi_{e}^{2}\right]
$$

To simplify notation we use $\xi_{e}$ to denote $\xi_{b}$ with $b=(0, e)$. Then our goal is prove the main Theorem of this section.

THEOREM 9.1 The following decomposition holds in $L_{2}(\mu)$ :

$$
\mathcal{G}_{C}=\overline{\mathcal{G}_{E}+\mathcal{G}^{(1)}}
$$

Together with Lemma 8.4 this proves Theorem 8.2. Theorem 9.1 has a parallel version if the conservative (Kawasaki) dynamics is replaced by a nonconservative (Glauber) one. For any configuration $\eta$ and $x \in \mathbb{Z}^{d}$ we define

$$
\eta_{y}^{x}=\left\{\begin{array}{lll}
\eta_{y} & \text { if } & x \neq y \\
1-\eta_{x} & \text { if } & x=y
\end{array}\right.
$$

i.e the spin is flipped at the site $x$. The Glauber gradient $\sigma_{x}$ at site $x$ of a function $u$ is defined as

$$
\left(\sigma_{x} u\right)(\eta)=u\left(\eta^{x}\right)-u(\eta)
$$

For any local function $g$, although the formal sum

$$
u=\sum_{z \in \mathbb{Z}^{d}} \tau_{z} g
$$

does not exist, the Glauber gradients

$$
\omega_{x}=\sigma_{x} u
$$

exist and are in fact local functions. Moreover we have the translational covariance

$$
\omega_{e}=\tau_{x} \omega_{x}
$$

along with the obvious identities

$$
\sigma_{x} \omega_{y}=\sigma_{y} \omega_{x} \quad \text { for all } \quad x \neq y
$$

and

$$
\sigma_{x} \omega_{x}=-2 \omega_{x} \quad \text { for all } \quad x \in \mathbb{Z}^{d}
$$

Let $\mathcal{G}_{C}^{G}$ be space of covariant closed forms (in the Glauber sense), i.e. $\left\{\omega_{x}\right\}$ that satisfy (9.4), (9.5) and (9.6). Equip $\mathcal{G}_{C}^{G}$ with the $L^{2}$ norm defined by

$$
\|\omega\|_{2}^{2}=E^{\mu}\left[\omega_{0}^{2}\right]
$$

Let $\mathcal{G}_{E}^{G}$ be the space of translationally covariant exact forms, namely

$$
\mathcal{G}_{E}^{G}=\left\{\omega: \omega_{x}=\sigma_{x} \sum_{z \in \mathbb{Z}^{d}} \tau_{y} g, g \text { is a local function }\right\} .
$$


We have the following approximation Theorem.

THEOREM 9.2 In $L^{2}(\mu)$ :

$$
\mathcal{G}_{C}^{G}=\overline{\mathcal{G}_{E}^{G}}
$$

Our interest in the Glauber dynamics arises from the observation that the spin flip and the spin exchange are related in the following manner. Given a pair of functions $u$ and $v$ let $\mathrm{Q}$ denote the function

$$
Q_{x, x+e}(u, v)=\left[\eta_{x}\left(1-\eta_{x+e}\right)+\eta_{x+e}\left(1-\eta_{x}\right)\right]\left[\sigma_{x+e} u+u+v\right]
$$

Then for any function $\mathrm{F}$,

$$
T_{x, x+e} F=Q_{x, x+e}\left(\sigma_{x} F, \sigma_{x+e} F\right) .
$$

We now prove Theorems 9.1 and 9.2. The first few steps of the proofs are very similar and the steps leading to Theorem 9.1 are harder. We shall provide the details for this case and just sketch the proofs for the easier version.

Proof. In general, given functions $\xi_{b}$ defined for bonds in a box $\Lambda_{\ell}$ satisfying the compatibility conditions for being a closed (Kawasaki) form, we can "integrate" it, and find a function $g$ such that

$$
\xi_{b}=T_{b} g \quad \text { for all } \quad b \in \Lambda_{\ell}
$$

The function $g$ is not unique, but can be determined uniquely by imposing a mean 0 condition

$$
E^{\mu_{\ell, \omega, y}}[g]=0
$$

that makes the solution depend on the external boundary condition $\omega$ and the particle density $y$ in $\Lambda_{\ell}$. For such a choice we have the estimate

$$
E^{\mu_{\ell, \omega, y}}\left[|g|^{2}\right] \leq C \ell^{2} E^{\mu_{\ell, \omega, y}}\left[\sum_{b \in \Lambda_{\ell}} \xi_{b}^{2}\right]
$$

as a consequence of the estimate on the spectral gap. The constant $C$ is of course independent of $\ell, \omega$ and $y$. We can perform this step even if $g$ depends on the external boundary condition $\omega$, so long as the compatibility is satisfied for each such $\omega$. There is a similar result on integrating Glauber forms $\left\{\xi_{x}: x \in \Lambda_{\ell}\right\}$ with the normalization $E^{\mu_{\ell, \omega, \lambda}}[g]=0$ along with the estimate

$$
E^{\mu_{\ell, \omega, \lambda}}\left[|g|^{2}\right] \leq C E^{\mu_{\ell, \omega, \lambda}}\left[\sum_{x \in \Lambda_{\ell}} \xi_{x}^{2}\right]
$$

again based on the spectral estimate, but now for the Glauber dynamics.

Let us recall that $R$ is the range of interaction in the Gibbs measure. For each box $\Lambda_{M}$ let $\partial \Lambda_{M}$ denote the "boundary" of $\Lambda_{M}$, consisting of sites in $\Lambda_{M}$ that are within a distance $R$ from some site in $\Lambda_{M}^{c}$ ( the complement of $\Lambda_{M}$ ) as well sites in $\Lambda_{M}^{c}$ that are within a distance $R$ of some site in $\Lambda_{M}$. Let $\Lambda_{M}^{0}=\Lambda_{M} \backslash \partial \Lambda_{M}$ denote the "interior" of $\Lambda_{M}$. Given a (Kawasaki) closed form $\left\{\xi_{b}\right\}$ we can try to truncate it over a finite volume $\Lambda_{M}$ by defining

$$
\xi_{b}^{M}=E^{\mu}\left[\xi_{b} \mid \mathcal{K}_{M}\right] \quad \text { for each } \quad b \in \Lambda_{M}
$$


While the conditioning interferes with the gradient near the boundary, if we restrict $\xi_{b}^{M}$ to $b \in \Lambda_{M}^{0}$, it does in fact define a (Kawasaki) closed form and in particular if $\xi_{b}=T_{b} g$ for some $g$, then $\xi_{b}^{M}=T_{b} g^{M}$ where $g^{M}=E^{\mu}\left[g \mid \mathcal{K}_{M}\right]$ so long as $b \in \Lambda_{M}^{0}$. The identical statement is valid for Glauber forms as well.

Suppose we are given a translationally covariant (Kawasaki) form $\left\{\xi_{b}\right\}$. For some $L \gg 1$ we define the truncations $\xi_{b}^{3 L}$ according to (9.13) for a box of size $3 L$. We can use these to construct a function $g_{3 L}$ measurable with respect to $\mathcal{K}_{3 L}$ such that $T_{b} g_{3 L}=\xi_{b}^{3 L}$ for $b \in \Lambda_{3 L}^{0}$. We then define

$$
h_{L}=E^{\mu}\left[g_{3 L} \mid \mathcal{K}_{L}\right]
$$

and finally an approximation that is translationally covariant and exact by

$$
\tilde{\xi}_{b}^{L}=\frac{1}{(2 L+1)^{d}} T_{b} \sum_{z \in \mathbb{Z}^{d}} \tau_{z} h_{L}
$$

In the Glauber context the approximation takes the form

$$
\bar{\omega}_{x}^{L}=\frac{1}{(2 L+1)^{d}} \sigma_{x} \sum_{z \in \mathbb{Z}^{d}} \tau_{z} h_{L}
$$

with a different but similarly constructed $h_{L}$.

LEMMA 9.3 The Kawasaki form $\tilde{\xi}_{e}^{L}$ can be decomposed as the sum $\tilde{\xi}_{e}^{L}=\tilde{\Omega}_{L, e}^{(1)}+\tilde{\Omega}_{L, e}^{(2)}$ coming respectively from the interior terms and the boundary terms. For each $e>0$, they satisfy

$$
\lim _{L \rightarrow \infty} E^{\mu}\left[\left|\xi_{e}-\tilde{\Omega}_{L, e}^{(1)}\right|^{2}\right]=0
$$

and

$$
\sup _{L} E^{\mu}\left[\left|\tilde{\Omega}_{L, e}^{(2)}\right|^{2}\right]<\infty
$$

The Glauber form $\bar{\omega}_{0}^{L}$ admits a similar decomposition as $\bar{\omega}_{0}^{L}=\bar{\Omega}_{L, 0}^{(1)}+\bar{\Omega}_{L, 0}^{(2)}$ coming from the interior terms and the boundary terms and they satisfy

$$
\lim _{L \rightarrow \infty} E^{\mu}\left[\left|\omega_{0}-\bar{\Omega}_{L, 0}^{(1)}\right|^{2}\right]=0
$$

and the stronger estimate

$$
\sup _{L} L E^{\mu}\left[\left|\bar{\Omega}_{L, 0}^{(2)}\right|^{2}\right]<\infty
$$

REMARK. In the Glauber case, from (9.16) and (9.17) it follows that $\bar{\omega}_{0}^{L} \rightarrow \omega_{0}$ as $L \rightarrow \infty$, and this proves Theorem 9.2. In the Kawasaki case we will take a weak limit of $\tilde{\Omega}_{L, 0}^{(2)}$ which will again be a covariant Kawasaki closed form and analyze it further and show that it is made up of density gradients. This will complete the proof of Theorem 9.1.

Proof. Denote by $\Omega^{(1)}$ the "interior part" and $\Omega^{(2)}$ the "boundary part", namely,

$$
\tilde{\Omega}_{L, e}^{(1)}=\frac{1}{(2 L+1)^{d}} T_{e} \sum_{y: y, y+e \in \Lambda_{L}^{0}} \tau_{y} h_{L}
$$




$$
\tilde{\Omega}_{L, e}^{(2)}=\frac{1}{(2 L+1)^{d}} T_{e} \sum_{y:\{y, y+e\} \cap \partial \Lambda_{L} \neq \phi} \tau_{y} h_{L}
$$

It is important to note at this point that, if $y, y+e \in \Lambda_{L}^{0}$ then

$$
T_{e} \tau_{y} h_{L}=\tau_{y} T_{y, y+e} h_{L}=\tau_{y} E^{\mu}\left[\xi_{y, y+e} \mid \mathcal{K}_{L}\right]
$$

Since $\xi_{e} \in L_{2}(\mu)$, for large $k, E^{\mu}\left[\left|\xi_{e}-E^{\mu}\left[\xi_{e} \mid \mathcal{K}_{k}\right]\right|^{2}\right]$ is small. By translational invariance $E^{\mu}\left[\left\{\xi_{\tau_{y} e}-E^{\mu}\left[\xi_{\tau_{y} e} \mid \mathcal{K}_{y, k}\right]\right\}^{2}\right]$ is independent of $y$ and is therefore uniformly small for large $k$. For most $y \in \Lambda_{L}, \Lambda_{y, k} \subset \Lambda_{L}^{0}$ and consequently for most bonds $b$ (with density close to 1 for large $M$ ), $E^{\mu}\left[\left(\xi_{b}-E^{\mu}\left[\xi_{b} \mid \mathcal{K}_{L}\right]\right)^{2}\right]$ is small. This proves (9.14). The proof of (9.16) is identical. The following lemma provides a key estimate.

LEMMA 9.4 Let $h$ be a function in the cube $\Lambda_{3 L}$. Suppose $\Lambda_{L}$ is the centered subcube of width $2 L+1, \bar{h}=E^{\mu}\left[h \mid \mathcal{K}_{L}\right]$ and $z$ is a site in $\Lambda_{L}$. Denote the configuration by $\eta=\left(\xi, \eta_{z}, \zeta\right)$ where $\zeta$ denote the configuration on $\Lambda_{L} \backslash\{z\}$ and $\xi$ denote the configuration on $\Lambda_{3 L} \backslash \Lambda_{L}$. Denote by $A v_{y}=A v_{y \in \Lambda_{3 L-R} \backslash \Lambda_{L+R}}$. Let $\mu$ be a Gibbs measure on $\Lambda_{3 L}$ or an infinite volume Gibbs state such that for any local function $F$ the following mixing property holds:

$$
E^{\mu}\left[A v_{y} F_{y} ; A v_{y} F_{y}\right] \leq C_{F} L^{-d}
$$

Then

$$
E^{\mu}\left[\left(\sigma_{z} \bar{h}\left(\eta_{z}, \zeta\right)\right)^{2}\right] \leq C L^{-d} E^{\mu}\left[h^{2}(\eta)\right]+C A v_{y} E^{\mu}\left[\left(T_{z, y} h\right)^{2}(\eta)\right]
$$

where the constant $C$ may depend on the density of the Gibbs measure.

We will defer the proof of the lemma to the end of the section and proceed with our main goal. We first consider the Glauber case.

By definition, $\bar{\Omega}_{L, 0}^{(2)}$ has contribution from the boundary consisting of

$$
\bar{\Omega}_{L, 0}^{(2)}=\frac{1}{(2 L+1)^{d}} \sigma_{0} \sum_{y \in \partial \Lambda_{L}} \tau_{y} h_{L}=\frac{1}{(2 L+1)^{d}} \sum_{y \in \partial \Lambda_{L}} \tau_{y} \sigma_{y} h_{L}
$$

and (9.17) will follow provided we show that

$$
\sup _{L} \sup _{y \in \Lambda_{L}} E^{\mu}\left[\left|\sigma_{y} h_{L}\right|^{2}\right] \leq C<\infty
$$

Clearly, (9.20) follows readily from (9.19) with the choice of $h=g_{3 L}$. We only have to note that in the Glauber case we have from the spectral gap bounds (9.12)

$$
E^{\mu}\left[g_{3 L}^{2}(\eta)\right] \leq C L^{d}
$$

and

$$
E^{\mu}\left[\left(T_{z, y} g_{3 L}\right)^{2}(\eta)\right] \leq C E^{\mu}\left[\left(\sigma_{z} g_{3 L}\right)^{2}(\eta)+\left(\sigma_{y} g_{3 L}\right)^{2}(\eta)\right] \leq C
$$

so long as $z$ and $y$ remain in $\Lambda_{3 L}^{0}$. This concludes the proof of Theorem 9.2

We now turn to the Kawasaki case. We observe that instead of (9.21) and (9.22) we obtain from (9.11)

$$
E^{\mu}\left[g_{3 L}^{2}(\eta)\right] \leq C L^{d+2}
$$


and

$$
E^{\mu}\left[\left(T_{z, y} g_{3 L}\right)^{2}(\eta)\right] \leq C L^{2}
$$

so long as $z$ and $y$ remain in $\Lambda_{3 L}^{0}$. This provides the estimate

$$
\sup _{y \in \Lambda_{L}} E^{\mu}\left[\left(\sigma_{y} h_{L}\right)^{2}(\eta)\right] \leq C L^{2}
$$

with a constant $C$ independent of $L$. Because the boundary terms are only $O\left(L^{d-1}\right)$ in number, from the definition of $\tilde{\Omega}_{L, \varepsilon}^{(2)}$ the estimate (9.25) is sufficient to establish (9.15). This concludes the proof of Lemma 9.3.

Return to the proof of Theorem 9.1. The proof is broken up into several steps.

Step 1. The boundary in $d$ dimensions comes in $2 d$ faces with some overlap at the corners. The overlap involves only $O\left(L^{d-2}\right)$ sites and hence negligible in view of the bound (9.25). We can therefore analyze the contribution from each face by itself. Since they are all similar we will just consider one of them. Depending on the range of the interaction, for some integer $q \geq 1$, the face consists of points $z=\left(z_{1}, \cdots, z_{d}\right) \in \mathbb{Z}^{d}$ satisfying $-L \leq z_{1} \leq-L+q$ and $-L \leq z_{i} \leq L$ for $2 \leq i \leq d$. The face consists naturally of layers

$$
B_{L}^{j}=\left\{z=\left(z_{1}, \cdots, z_{d}\right): z_{1}=-L+j ;-L \leq z_{i} \leq L \text { for } 2 \leq i \leq d\right\}
$$

indexed by $0 \leq j \leq q$. We need to analyze the terms

$$
\xi_{e}^{B, L}=\frac{1}{(2 L+1)^{d}} \sum_{z \in B_{L}} \tau_{z} T_{z, z+e} h_{L}=\frac{1}{(2 L+1)^{d}} T_{e} \sum_{z \in B_{L}} \tau_{z} h_{L}
$$

where $B_{L}$ is the slightly thickened boundary

$$
B_{L}=\cup_{-1 \leq j \leq q} B_{L}^{j}
$$

We can rewrite

$$
\xi_{e}^{B, L}=T_{e} \sum_{-1 \leq j \leq q} \tau_{j}^{1} \bar{h}_{L}
$$

where

$$
\bar{h}_{L}=\frac{1}{(2 L+1)^{d}} \sum_{z \in B_{L}^{0}} \tau_{z} h_{L}
$$

and $\tau_{j}^{1}=\tau_{j e_{1}}$ is the translation by $j$ steps in the $x_{1}$ direction. For each site in $x=\left(x_{1}, \cdots, x_{d}\right) \in \mathbb{Z}^{d}$ let us consider

$$
\omega_{x}^{B, L}=\sigma_{x} \bar{h}_{L}
$$

Since $\omega_{x}^{B, L}$ is bounded in $L_{2}(\mu)$, we can take a weak limit as $L \rightarrow \infty$, with one subsequence working simultaneously for all $x$, and call the limt $\omega_{x}^{B}$.

Step 2. By construction, as a weak limit of Glauber gradients, $\left\{\omega_{x}^{B}\right\}$ is again a Glauber gradient although it may not be covariant. Because averaging is done in all directions except in the first coordinate direction, it is covariant, in all directions except possibly in that direction. Moreover $\omega_{x}^{B}=0$ if $x_{1}<0$, because none of the functions depend on the configuration at any site $x$ with $x_{1}<0$. Let $b$ be a bond that does not intersect the infinite $\operatorname{strip} B_{\infty}=\left\{y=\left(y_{1}, \cdots, y_{d}\right): 0 \leq y_{1} \leq q\right\}$, as well as 
the site $x$. Then $T_{b} \bar{h}_{L} \rightarrow 0$, and $\sigma_{x}$ commutes with $T_{b}$. Therefore $T_{b} \omega_{x}^{B}=0$. This implies that $\omega_{x}^{B}$ can only depend on the configuration in $B_{\infty} \cup\{x\}$. In particular for $y \notin B_{\infty} \cup\{x\}, \sigma_{y} \omega_{x}^{B}=0$. As a Glauber gradient $\sigma_{y} \omega_{x}^{B}=\sigma_{x} \omega_{y}^{B}$ for all $x, y$ and hence we also have $\sigma_{x} \omega_{y}^{B}=0$ for such pairs $x$ and $y$. It is easy to arrive at the following conclusion: for each $x \in B_{\infty}, \omega_{x}^{B}$ depends only on the configuration in $B_{\infty}$. On the other hand for $x$ with $x_{1}>q, \omega_{x}^{B}$ can only depend on $\eta_{x}$ and as a gradient must equal $c(x)\left(2 \eta_{x}-1\right)$ for some constant $c(x)$. Covariance in all directions except the first coordinate means that $c(x)$ is a function of the first coordinate only i.e $c(x)=c\left(x_{1}\right)$

Step 3. If we denote by $\xi_{e}^{B}$ the weak limit of $\xi_{e}^{B, L}$, then

$$
\begin{array}{r}
\xi_{e}^{B}=\lim _{L \rightarrow \infty}\left[\eta_{0}\left(1-\eta_{e}\right)+\eta_{e}\left(1-\eta_{0}\right)\right]\left[\left(\sigma_{0} \sigma_{e}+\sigma_{0}+\sigma_{e}\right)\left(\sum_{-1 \leq j \leq q} \tau_{j}^{1} \bar{h}_{L}\right)\right] \\
=\lim _{L \rightarrow \infty}\left[\eta_{0}\left(1-\eta_{e}\right)+\eta_{e}\left(1-\eta_{0}\right)\right]\left[\sigma_{0}\left(\sum_{-1 \leq j \leq q} \tau_{j}^{1} \sigma_{e+j e_{1}} \bar{h}_{L}\right)+\left(\sum_{-1 \leq j \leq q} \tau_{j}^{1} \sigma_{j e_{1}} \bar{h}_{L}\right)\right. \\
\left.+\left(\sum_{-1 \leq j \leq q} \tau_{j}^{1} \sigma_{e+j e_{1}} \bar{h}_{L}\right)\right] \\
=\left[\eta_{0}\left(1-\eta_{e}\right)+\eta_{e}\left(1-\eta_{0}\right)\right]\left[\sigma_{0}\left(\sum_{-1 \leq j \leq q} \tau_{j}^{1} \omega_{e+j e_{1}}^{B}\right)+\left(\sum_{-1 \leq j \leq q} \tau_{j}^{1} \omega_{e+j e_{1}}^{B}\right)\right. \\
\left.+\left(\sum_{-1 \leq j \leq q} \tau_{j}^{1} \omega_{j e_{1}}^{B}\right)\right]
\end{array}
$$

If $\omega_{x}$ is a Glauber gradient then

$$
\bar{\omega}_{x}=\sum_{z \in \mathbb{Z}^{d}} \tau_{z} \omega_{x+z}
$$

is a covariant Glauber gradient. If $\omega_{x}$ is covariant in all directions except the first, we can redefine

$$
\bar{\omega}_{x}=\sum_{j} \tau_{j}^{1} \omega_{x+j e_{1}}
$$

and get a covariant Glauber gradient. Let us define a new Glauber gradient

$$
\tilde{\omega}_{x}^{B}=\omega_{x}^{B}-c\left(x_{1}\right)\left(2 \eta_{x}-1\right)
$$

where $c\left(x_{1}\right)$ is defined in Step 2 for $x_{1}>q$ and is taken to be 0 otherwise. Then

$$
\bar{\omega}_{x}^{B}=\sum_{j} \tau_{j}^{1} \tilde{\omega}_{x+j e_{1}}^{B}
$$

is a covariant Glauber gradient and so can be approximated by exact Glauber gradients according to Theorem 9.2. Therefore the Kawasaki gradient

$$
\bar{\xi}_{e}^{B}=\left[\eta_{0}\left(1-\eta_{e}\right)+\eta_{e}\left(1-\eta_{0}\right)\right]\left[\sigma_{e} \bar{\omega}_{0}^{B}+\bar{\omega}_{0}^{B}+\bar{\omega}_{e}^{B}\right]
$$

is covariant and can also be approximated by exact forms. We now calculate the difference

$$
\xi_{e}^{B}-\bar{\xi}_{e}^{B}=\left[\eta_{0}\left(1-\eta_{e}\right)+\eta_{e}\left(1-\eta_{0}\right)\right]\left[\sigma_{0}\left(u^{B}-\bar{\omega}_{0}^{B}\right)+\left(u^{B}-\bar{\omega}_{0}^{B}\right)+\left(v^{B}-\bar{\omega}_{e}^{B}\right)\right]
$$


where

$$
\begin{aligned}
u^{B} & =\sum_{-1 \leq j \leq q} \tau_{j}^{1} \omega_{j e_{1}}^{B} \\
\bar{\omega}_{0}^{B} & =\sum_{j} \tau_{j}^{1}\left[\omega_{j e_{1}}^{B}-c_{j}\left(2 \eta_{j e_{1}}-1\right)\right] \\
v^{B} & =\sum_{-1 \leq j \leq q} \tau_{j}^{1} \omega_{e+j e_{1}}^{B} \\
\bar{\omega}_{e}^{B} & =\sum_{j} \tau_{j}^{1}\left[\omega_{e+j e_{1}}^{B}-c_{j+\delta_{e, e_{1}}}\left(2 \eta_{e+j e_{1}}-1\right)\right]
\end{aligned}
$$

A straight forward calculation reveals that $u^{B}=\bar{\omega}_{0}^{B}$ and if $e \neq e_{1}$ we also have $v^{B}=\bar{\omega}_{e}^{B}$. However if $e=e_{1}, v^{B}-\bar{\omega}_{e_{1}}^{B}=c_{j+1}\left(2 \eta_{e_{1}}-1\right)$ and then $\xi_{e_{1}}^{B}-\bar{\xi}_{e_{1}}^{B}$ is seen to equal $c \nabla_{e_{1}} \eta_{0}$, a multiple of the density gradient. This completes the proof of Theorem 9.2 .

Proof of Lemma 9.4. By definition,

$$
\bar{h}\left(\eta_{z}, \zeta\right)=E^{\mu}\left[h \mid \eta_{z}, \zeta\right]=Z\left(\eta_{z}, \zeta\right)^{-1} \sum_{\xi} h(\eta) \exp [-H(\eta)]
$$

where $Z\left(\eta_{z}, \zeta\right)=\sum_{\xi} \exp \left[-H\left(\xi, \eta_{z}, \zeta\right)\right]$ is the partition function. Let

$$
\bar{\xi}=A v_{y \in \Lambda_{3 L-R} \backslash \Lambda_{L+R}} \xi_{y}
$$

and denote $E^{\mu}\left[\bar{\xi} \mid \eta_{z}, \zeta\right]$ by $\rho\left(\eta_{z}, \zeta\right)$. From the definition of truncated correlation function, we have $\bar{h}\left(\eta_{z}, \zeta\right)=B_{2}\left(\eta_{z}, \zeta\right)-B_{1}\left(\eta_{z}, \zeta\right)$ where

$$
\begin{aligned}
& B_{1}\left(\eta_{z}, \zeta\right)=\rho\left(\eta_{z}, \zeta\right)^{-1} E^{\mu}\left[h ; \bar{\xi} \mid \eta_{z}, \zeta\right] \\
& B_{2}\left(\eta_{z}, \zeta\right)=\rho\left(\eta_{z}, \zeta\right)^{-1} E^{\mu}\left[h \bar{\xi} \mid \eta_{z}, \zeta\right]
\end{aligned}
$$

Explicitly,

$$
B_{2}(0, \zeta)=\rho(0, \zeta)^{-1} Z(0, \zeta) \sum_{\xi} \bar{\xi} h(\xi, 0, \zeta) \exp [-H(\xi, 0, \zeta)]
$$

Replacing $h(\xi, 0, \zeta)$ by $A v_{y} \xi_{y} h\left(\sigma_{y} \xi, 1, \zeta\right)$, we have $B_{2}(0, \zeta)=B_{3}+B_{4}$ where

$$
\begin{aligned}
& B_{3}(\zeta)=\rho(0, \zeta)^{-1} Z(0, \zeta)^{-1} \sum_{\xi} A v_{y} \xi_{y}\left[h(\xi, 0, \zeta)-h\left(\sigma_{y} \xi, 1, \zeta\right)\right] \exp [-H(\xi, 0, \zeta)] \\
& B_{4}(\zeta)=\rho(0, \zeta)^{-1} Z(0, \zeta)^{-1} \sum_{\xi} A v_{y} \xi_{y} h\left(\sigma_{y} \xi, 1, \zeta\right) \exp [-H(\xi, 0, \zeta)]
\end{aligned}
$$

By definition,

$$
\left(\xi_{y}\left[h(\xi, 0, \zeta)-h\left(\sigma_{y} \xi, 1, \zeta\right)\right]\right)^{2} \leq\left(T_{z, y} h\right)(\xi, 0, \zeta)^{2}
$$

Hence we can bound $B_{3}$ by

$$
E^{\mu}\left[\left(B_{3}(\zeta)\right)^{2}\right] \leq C A v_{y} E^{\mu}\left[\left(T_{z, y} h\right)(\eta)^{2}\right]
$$


By changing the variable $\sigma_{y} \xi \rightarrow \xi, B_{4}$ equals to

$$
\begin{aligned}
B_{4}(\zeta) & =\rho(0, \zeta)^{-1} Z(1, \zeta)^{-1} \sum_{\xi}\left[\left(A v_{y} F_{y}\right) h(\xi, 1, \zeta)\right] \exp [-H(\xi, 1, \zeta)] \\
& =\rho(0, \zeta)^{-1} E^{\mu}\left[\left(A v_{y} F_{y}\right) h \mid 1, \zeta\right]
\end{aligned}
$$

where $F_{y}$ is the local function

$$
F_{y}=\exp \left[H(\xi, 1, \zeta)-H\left(\sigma_{y} \xi, 0, \zeta\right)\right]
$$

and Because the sites $y$ and $z$ are sufficiently apart $G$ does not depend on $y$. By definition of the corrected correlation function,

$$
E^{\mu}\left[\left(A v_{y} F_{y}\right) h \mid 1, \zeta\right]=E^{\mu}\left[\left(A v_{y} F_{y}\right) ; h \mid 1, \zeta\right]+E^{\mu}\left[\left(A v_{y} F_{y}\right) \mid 1, \zeta\right] E^{\mu}[h \mid 1, \zeta]
$$

To compute $E^{\mu}\left[\left(A v_{y} F_{y}\right) \mid 1, \zeta\right]$, consider $h$ independent of $\xi$. From $(9.26), B_{4}(\zeta)=$ $h(1, \zeta)$. From $(9.28)$ and $(9.29), B_{4}(\zeta)=\rho(0, \zeta)^{-1} E^{\mu}\left[\left(A v_{y} F_{y}\right) \mid 1, \zeta\right] h(1, \zeta)$. Hence $\rho(0, \zeta)^{-1} E^{\mu}\left[\left(A v_{y} F_{y}\right) \mid 1, \zeta\right]=1$ and thus

$$
B_{4}(\zeta)=\rho(0, \zeta)^{-1} E^{\mu}\left[\left(A v_{y} F_{y}\right) ; h \mid 1, \zeta\right]+\bar{h}(1, \zeta)
$$

We have thus proved that

$$
\bar{h}(0, \zeta)-\bar{h}(1, \zeta)=-B_{1}(0, \zeta)+B_{3}(\zeta)+\rho(0, \zeta)^{-1} E^{\mu}\left[\left(A v_{y} F_{y}\right) ; h \mid 1, \zeta\right]
$$

From the mixing assumption (9.18) the last term on the right hand side satisfies the bound

$$
\begin{aligned}
E^{\mu} & \left\{E^{\mu}\left[\left(A v_{y} F_{y}\right) ; h \mid 1, \zeta\right]^{2}\right\} \\
& \leq C E^{\mu}\left\{E^{\mu}\left[\left(A v_{y} F_{y}\right) ;\left(A v_{y} F_{y}\right) \mid 1, \zeta\right]^{2}\right\} E^{\mu}\left\{E^{\mu}\left[h^{2} \mid(1, \zeta)\right]\right\} \\
& \leq C L^{-d} E^{\mu}\left[h^{2}\right]
\end{aligned}
$$

Also, $B_{1}$ satisfies a similar bound. Together with (9.27), this concludes Lemma 9.4.

10. Mixing properties of grand canonical and canonical gibbs measures. Let us recall our basic mixing condition.

Assumption A. Let $\mu_{\Lambda, \omega, \lambda}$ denote a Gibbs measure on $\Lambda$ with boundary condition $\omega$ and chemical potential $\lambda$. Denote the corresponding density by $\rho=\rho(L, \lambda, \omega)$. Then there are constants $\gamma_{1}, \gamma_{2}$ and $\gamma_{3} \geq R+1$ such that for any two functions $f$ and $g$ with supports $S_{f}$ and $S_{g}$ we have

$$
\left|E_{\Lambda, \omega, \lambda}[f ; g]\right| \leq \gamma_{1} \rho(1-\rho) \exp \left[-\gamma_{2} \operatorname{dist}\left(S_{f}, S_{g}\right)\right]\|f\|_{\infty}\|g\|_{\infty}
$$

provided that the diameters of $S_{f}$ and $S_{g}$ are bounded by $\gamma_{3}$. Note that the constants are independent of the size $L$ of the cube $\Lambda$, the value of $\lambda$ of the chemical potential and the boundary condition $\omega$.

REMARK. The term $\rho(1-\rho)$ is effective only for densities $\rho$ that are close to 0 or 1. The effect of $L$ and $\omega$ is minimal. In fact for any local interaction, by low density cluster expansion, (10.1) is seen to be always satisfied provided $|\lambda|$ is sufficiently large. The factor $\rho(1-\rho)$ in $(10.1)$ is therefore available for free.

From this assumption, one immediately obtains the following lemma. 
Lemma 10.1 There is a positive function $C$ on $\mathbb{Z}$ such that the following holds. Suppose $\omega$ is a boundary conditions on $\partial \Lambda$ and $z \in \partial \Lambda$. For any $g$ in $\Lambda$,

$$
\left|E_{\Lambda, \omega, \lambda}[g]-E_{\Lambda, \sigma_{z} \omega, \lambda}[g]\right| \leq C\left(\left|S_{g}\right|\right) \exp \left[-\gamma_{2} \operatorname{dist}\left(z, S_{g}\right)\right]\|g\|_{\infty} .
$$

where $\sigma_{z} \omega$ denote the boundary condition obtained by changing the configuration at the site $z$ and $\left|S_{g}\right|$ denotes the number of the sites in $S_{g}$.

Furthermore, there is a universal function $C\left(\left|S_{f}\right|,\left|S_{g}\right|\right)$ such that (10.1) holds with no restriction on the diameter of $S_{f}$ or $S_{g}$ provided that the constant $\gamma_{1}$ is replaced by $C\left(\left|S_{f}\right|,\left|S_{g}\right|\right)$.

We now sketch a proof of this lemma. By definition, $\left|E_{\Lambda, \omega, \lambda}[g]-E_{\Lambda, \sigma_{z} \omega, \lambda}[g]\right|$ can be estimated by correlation between $g$ and a local function around the site $z$. Hence (10.2) follows from (10.1) provided that the diameter of the support $g$ is bounded by $\gamma_{3}$. Next, we prove (10.1) if the diameter of $S_{g}$ is bounded by $\gamma_{3}$. Let $\Omega \subset \Lambda \backslash S_{f}$ be a cube of size smaller than $2 \operatorname{dist}\left(S_{f}, S_{g}\right)$ and containing $S_{g}$ such that $\operatorname{dist}\left(\partial \Omega \backslash \partial \Lambda, S_{f}\right) \geq$ $\operatorname{dist}\left(S_{f}, S_{g}\right)-2$. Then

$$
E_{\Lambda, \omega, \lambda}[f ; g]=E_{\Lambda, \omega, \lambda}\left[f ; E_{\Lambda, \omega, \lambda}\left[g \mid \eta_{x}, x \in \Lambda \backslash \Omega\right]\right]
$$

Since the diameter of $S_{g}$ is bounded by $\gamma_{3}$, we can apply the estimate (10.2). Hence $E_{\Lambda, \omega, \lambda}\left[g \mid \eta_{x}, x \in \Lambda \backslash \Omega\right]$ is independent of the boundary condition $\eta_{x}, x \in \Lambda \backslash \Omega$, up to exponentially small error. This proves (10.1). Repeating the argument in the beginning of the sketch, we have (10.2) holds without restriction on the diameter of the support $S_{g}$. It follows that (10.1) holds without restriction on $S_{f}$ or $S_{g}$ by repeating the previous argument.

We now recall some of the notation that will be used in this section. We denote by $\Lambda_{L}$ a cube of width $2 L+1$. The (grand canonical) Gibbs measure with the chemical potential $\lambda$ and boundary condition $\omega$ is denoted by $\mu_{L, \omega, \lambda}$. If, instead of fixing the chemical potential $\lambda$, we fix the number of particles $N$ in $\Lambda_{L}$ or equivalently the density $y=\bar{\eta}=N L^{-d}$ in $\Lambda_{L}$, the resulting canonical measure is denoted by $\mu_{L, \omega, y}$. We denote by $M=(2 L+1)^{d}=\left|\Lambda_{L}\right|$. Using the elementary Fourier analysis the canonical measure $\mu_{L, \omega, y}$ can be represented as

$$
d \mu_{L, \omega, y}=\frac{1}{p_{y}}\left\{\frac{1}{2 \pi} \int_{-\pi}^{\pi} \exp [i \theta M(\bar{\eta}-y)] d \mu_{L, \omega, \lambda} d \theta\right\}
$$

where

$$
p_{y}=\mu_{L, \omega, \lambda}[\bar{\eta}=y]
$$

By definition, $M \bar{\eta}$ is an integer and thus $M y$ has to be an integer for $\mu_{L, \omega, y}$ to be meaningful. We shall always assume this to be the case. The chemical potential $\lambda$ is usually chosen such that

$$
E^{\mu_{L, \omega, \lambda}}[\bar{\eta}]=y+o(1)
$$

where $\lim \sup _{L \rightarrow \infty} o(1)=0$. We shall make the choice of $\lambda$ so that the right hand side is actually equal to $y$.

The boundary conditions will be fixed in this section and we will drop the subscript $\omega$. The main result of this section is the following Lemma.

LemMA 10.1 Suppose $\Lambda_{L}$ is a cube of width $2 L+1$ centered at 0 . Let the mixing condition (10.1) hold.. Then for any $s>0$, there is a constant $C_{s}$ such that the following estimate holds

$$
\sup _{\omega} \sup _{y}\left|E^{\mu_{L, \omega, y}}\left[\eta_{0}-\eta_{e}\right]\right|<C_{s} L^{-s}
$$


for any $e>0$.

A version of Lemmas 10.1 was proved in [Y2] using expansion techniques in local limit theorems, for $s \leq 3 / 2$. Our proof, although partly based on this approach, is somewhat simpler and yields a stronger result in the special case considered here. Since a local limit theorem is proved in [Y2], we have in particular

$$
p_{y} \geq C(y) L^{-d / 2}
$$

under the mixing assumption (10.1) . Here the chemical potential $\lambda$ is chosen such that (10.5) holds without the error term $o(1)$. We will need a lower bound that holds uniformly in the range $1-L^{\varepsilon-d} \geq y \geq L^{\varepsilon-d}$. Since, in principle, the lower bound should get better at the edges we will provide a simple proof of a uniform version of this estimate while proving Lemma 10.1 below.

Proof of Lemma 10.1. Case 1: $1-L^{\varepsilon-d} \geq y \geq L^{\varepsilon-d}$.

Step 1: Cutoff.

By definition, the expectation w.r.t. the canonical measure is given by

$$
E^{\mu_{L, y}}\left[\left(\eta_{0}-\eta_{e}\right)\right]=\frac{1}{p_{y}} \frac{1}{2 \pi} \int_{-\pi}^{\pi} E^{\mu_{L, \lambda}}\left\{\left(\eta_{0}-\eta_{e}\right) \exp [i \theta M(\bar{\eta}-y)]\right\} d \theta
$$

We first prove that the contribution to the integral from the range $\left\{\theta:|\theta| \geq L^{-\delta}\right\}$ is exponentially small, provided $4 \delta<\varepsilon$. Let $\Gamma$ be such that it contains 0 as well as $e$, and for some positive constant $c, \Lambda \backslash \Gamma$ consists of $c M$ widely separated sites that become independent when conditioned on $\Gamma \cup \Lambda_{L}^{c}$. Since

$$
\begin{aligned}
\mid E^{\mu_{L, \lambda}}\left\{\left(\eta_{0}-\eta_{e}\right)\right. & \exp [i \theta M(\bar{\eta}-y)]\}|=| E^{\mu_{L, \lambda}}\left\{\left(\eta_{0}-\eta_{e}\right) \exp \left[i \theta \sum_{x \in \Lambda_{L}} \eta_{x}\right]\right\} \mid \\
& =\mid E^{\mu_{L, \lambda}}\left\{\left(\eta_{0}-\eta_{e}\right) \exp \left[i \theta \sum_{x \in \Gamma} \eta_{x}\right] E^{\mu_{\Gamma, \lambda}}\left\{\exp \left[i \theta \sum_{x \in \Lambda \backslash \Gamma} \eta_{x}\right\}\right\} \mid\right. \\
& \leq \sup _{\omega} \mid E^{\mu_{\Gamma, \lambda}}\left\{\exp \left[i \theta \sum_{x \in \Lambda \backslash \Gamma} \eta_{x}\right\} \mid\right.
\end{aligned}
$$

it is enough to prove that

$$
\mid E^{\mu_{\Gamma, \lambda}}\left\{\exp \left[i \theta \sum_{x \in \Lambda \backslash \Gamma} \eta_{x}\right\} \mid \leq \exp \left(-C L^{\alpha}\right)\right.
$$

for the expectation with respect $\mu_{\Gamma, \lambda}$, the distribution obtained by conditioning on the configuration in the domain $\Gamma$. Let $p_{x}^{\Gamma}=E^{\mu_{\Gamma, \lambda}}\left[\eta_{x}\right]$. Since the interaction between $\eta_{x}$ and its neighbors is uniformly bounded for each fixed $x$, there is a constant $C$ such that

$$
C^{-1} \leq \frac{p_{x}^{\Gamma}}{y} \leq C \quad \text { and } \quad C^{-1} \leq \frac{1-p_{x}^{\Gamma}}{1-y} \leq C
$$

where $y$ is the total density. Note that this bound is independent of the shape of $\Gamma$. Since under $\mu_{\Gamma, \lambda},\left\{\eta_{x}\right\}$ are independent random variables one has

$$
E^{\mu_{\Gamma, \lambda}}\left[\exp \left[\sum_{x \in \Lambda \backslash \Gamma} i \theta \eta_{x}\right]\right]=\prod_{x \Lambda \backslash \Gamma}\left[p_{x}^{\Gamma} e^{i \theta}+\left(1-p_{x}^{\Gamma}\right)\right] .
$$


For any $0 \leq a \leq 1$ and $-\pi \leq \theta \leq \pi$ we have the elementary bound

$$
\left|a e^{i \theta}+1-a\right|^{2}=1-2 a(1-a)(1-\cos \theta) \leq \exp \left(-a(1-a) \theta^{2}\right) .
$$

We have thus proved

$$
\left|E^{\mu_{L, \lambda}}\left\{\left(\eta_{0}-\eta_{e}\right) \exp [i \theta M(\bar{\eta}-y)]\right\}\right| \leq \exp \left[-C^{-1} L^{\varepsilon-d} c M L^{-2 \delta}\right]=\exp \left[-c C^{-1} L^{\varepsilon-2 \delta}\right]
$$

Step 2. We can now assume that

$$
|\theta| \leq L^{-\delta}
$$

Choose a cube $I$ of width $L^{\alpha}$ with $\alpha$ to be chosen depending on $\varepsilon$ and centered at 0 . Hence

$$
\begin{aligned}
& E^{\mu_{L, \lambda}}\left\{\left(\eta_{0}-\eta_{e}\right) \exp \left[i \theta \sum_{x \in \Lambda_{L}}\left(\eta_{x}-y\right)\right]\right\} \\
& =E^{\mu_{L, \lambda}}\left\{\exp \left[i \theta \sum_{x \in I^{c}}\left(\eta_{x}-y\right)\right] U(\eta)\right\}
\end{aligned}
$$

where

$$
U(\eta)=E^{\mu_{L, \lambda}}\left[\left(\eta_{0}-\eta_{e}\right) \exp \left(i \theta \sum_{x \in I}\left(\eta_{x}-y\right)\right) \mid \eta_{x}, x \in I^{c}\right]
$$

From the Taylor expansion and the bound (10.10) on $\theta$, for any integer $\ell$, we have

$$
U(\eta)=\sum_{j=0}^{\ell} U_{j}(\eta)+O\left(L^{-(\ell+1)(\delta-d \alpha)}\right)
$$

where

$$
U_{j}(\eta)=\frac{(i \theta)^{j}}{j !} \sum_{x_{i} \in I, i=1, \cdots, j} E^{\mu_{L, \lambda}}\left[\left(\eta_{x_{1}}-y\right) \ldots\left(\eta_{x_{j}}-y\right)\left(\eta_{0}-\eta_{e}\right) \mid I^{c}\right]
$$

We claim that

$$
\sup _{\eta}\left|U_{i}(\eta)\right| \leq C \exp \left[-L^{-\beta}\right]
$$

for some $\beta>0$.

Step 3. We now prove this bound, e.g., for $j=\ell$. Given a box $\Lambda_{L}$, we pick an integer $\gamma$ such that $L \geq(\ell+1) \gamma$ and consider boxes $\Lambda^{j}=\{x:|x| \leq j \gamma\}$ for $1 \leq j \leq \ell$, and $\Lambda^{\ell+1}=\Lambda_{L}$. We have $(\ell+1)$ annuli of width at least $\gamma$. Given $\ell$ sites $x_{1}, \cdots, x_{\ell}$ at least one annuli $A_{j}=\Lambda^{j} \backslash \Lambda^{j-1}$ is empty. Let us write the set of all possible configurations as a disjoint union of $B_{1}, \cdots, B_{\ell+1}$ where $B_{j}$ is all configurations with $A_{j}$ having no points and each $A_{i}$ for $i>j$ having at least one site from among $x_{1}, \cdots, x_{\ell}$. We fix the boundary condition $\omega$ and denote by

$$
f_{L}^{\omega}\left(x, x_{1}, \cdots, x_{\ell}\right)=E^{L, \omega}\left[\left(\eta_{x}-y\right)\left(\eta_{x_{1}}-y\right) \cdots\left(\eta_{x_{\ell}}-y\right)\right]
$$

for $x=0, e$ and $x_{1}, \cdots, x_{\ell} \in \Lambda_{L}$. Our goal is to estimate the sum

$$
\Delta(\omega, L)=\sum_{x_{1}, \cdots, x_{\ell} \in \Lambda_{L}}\left[f_{L}^{\omega}\left(1, x_{1}, \cdots, x_{\ell}\right)-f_{L}^{\omega}\left(0, x_{1}, \cdots, x_{\ell}\right)\right]
$$


and show that it decays uniformly in $\omega$ faster than a stretched exponential in $L$. It is clearly sufficient to show that every $j \leq \ell+1$

$$
\Delta_{j}(\omega, L)=\sum_{x_{1}, \cdots, x_{\ell} \in B_{j}}\left[f_{L}^{\omega}\left(1, x_{1}, \cdots, x_{\ell}\right)-f_{L}^{\omega}\left(0, x_{1}, \cdots, x_{\ell}\right)\right]
$$

has the same property. The set $B_{j}$ has no sites in the $j$ th annulus and can further be split up in to $\ell+1$ sets depending on how many sites are inside and how many are outside. We denote the infinite volume expectations by

$$
f\left(x, x_{1}, \cdots, x_{\ell}\right)=E\left[\eta_{x} \eta_{x_{1}} \cdots \eta_{x_{\ell}}\right]
$$

If there are $m$ inside sites $y_{1} \cdots, y_{m}$ and $n$ outside variables $z_{1}, \cdots, z_{n}$ with $\ell=m+n$ and $\left(x_{1}, \cdots, x_{\ell}\right)=\left(y_{1}, \cdots, y_{m}, z_{1}, \cdots, z_{n}\right)$, from the mixing conditions it follows that

$$
f_{L}^{\omega}\left(x, x_{1}, \cdots, x_{\ell}\right)-f\left(x, y_{1} \cdots, y_{m}\right) f_{L}^{\omega}\left(z_{1}, \cdots, z_{n}\right)
$$

is exponentially small. Hence up to exponential small error we have

$$
\Delta_{j}(\omega, L) \sim \sum_{y_{1}, \cdots, y_{m} \in \Lambda_{j-1}}\left[f\left(1, y_{1} \cdots, y_{m}\right)-f\left(0, y_{1} \cdots, y_{m}\right)\right] \sum_{z_{1}, \cdots, z_{n} \in \Lambda \backslash \Lambda_{j}} f_{L}^{\omega}\left(z_{1}, \cdots, z_{n}\right)
$$

Since the number of terms is only polynomial in $L$, it follows that our task can be achieved if show that for every $L$ the sum

$$
\Delta(L)=\sum_{x_{1}, \cdots, x_{\ell} \in \Lambda_{L}}\left[f\left(1, x_{1}, \cdots, x_{\ell}\right)-f\left(0, x_{1}, \cdots, x_{\ell}\right)\right]
$$

is exponentially small. We prove this by induction on $\ell$ and we use the completely corrected correlation functions.

Recall that the definition of the completely corrected correlation function can be defined inductively by

$$
g_{k}\left(x_{1}, \cdots, x_{k}\right)=f_{k}\left(x_{1}, \cdots, x_{k}\right)-\sum_{A, B} g_{|A|}(A) g_{|B|}(B)
$$

where $A, B$ is a proper partition of the variables $x_{1}, \cdots, x_{k}$ into two disjoint subsets with $A$ and $B$ nonempty. It is well-known that under the mixing assumption $\mathrm{A}$ the function $g$ decays in all directions, i.e.,

$$
\left|g_{k}\left(x_{1}, \cdots, x_{k}\right)\right| \leq C e^{-c\left|x_{i}-x_{j}\right|}
$$

for all $i, j$. Since $f$ is expressed in terms of $g$, it suffices to prove that $\Delta(L)$ is exponentially small if $f$ is replaced by $g_{k}$ for $k \leq \ell+1$. Since $g_{k}\left(x, x_{1}, \cdots, x_{k}\right)$ is exponentially small unless $\left|x_{j}\right| \leq L^{\epsilon}$ for some $\epsilon>0$, we can drop the restriction that $x_{i} \in \Lambda_{L}$ because the added terms are exponentially small. But from the translational invariance of the Gibbs state, we have

$$
\sum_{x_{1}, \cdots, x_{k}}\left[g_{k}\left(1, x_{1}, \cdots, x_{k}\right)-g_{k}\left(0, x_{1}, \cdots, x_{k}\right)\right]=0
$$


This concludes the proof.

Step 4. Lower bound.

Let $y$ be given in the range $1-L^{\varepsilon-d} \geq y \geq L^{\varepsilon-d}$ and $\lambda=\lambda(L, y, \omega)$ be picked to satisfy

$$
E^{\mu_{\Lambda, \lambda, \omega}}\left\{M^{-1} \sum_{x \in \Lambda_{L}} \eta_{x}\right\}=y .
$$

Let find a set $\Gamma$ such that conditioning on $\Gamma \cup \Lambda^{c}$ makes $\left\{\eta_{x}: x \in \Lambda \backslash \Gamma\right\}$ mutually independent. The cardinality of $\Lambda \backslash \Gamma$ is about $c M$ for some $c>0$. Let us denote by $n_{\Gamma}$ and $n_{\Gamma^{c}}$ the number of particles in $\Gamma$ and $\Gamma^{c}$ respectively and for $x \in \Gamma^{c}$ by $p_{x}^{\Gamma}$ the conditional probability that $\eta_{x}=1$, given the configuration in $\Gamma \cup \Lambda^{c}$. By a conditioning argument

$$
\mu_{\Lambda, \lambda, \omega}\{\bar{\eta}=y\}=E^{\mu_{\Lambda, \lambda, \omega}}\left\{\mu_{\Lambda, \lambda, \omega}\left\{n_{\Gamma^{c}}=M y-n_{\Gamma} \mid \Gamma \cup \Lambda^{c}\right\}\right\}
$$

The quantity $n_{\Gamma c}$ is a sum of independent Bernoulli random variables with expectations $p_{x}^{\Gamma}$ that satisfy $c y \leq p_{x}^{\Gamma} \leq C y$, one can easily derive the standard local limit theorem that provides the lower bound

$$
\mu_{\Lambda, \lambda, \omega}\left\{n_{\Gamma^{c}}=M y-n_{\Gamma} \mid \Gamma \cup \Lambda^{c}\right\} \geq \frac{c}{\sigma_{L}(\omega)} \exp \left[-\frac{\left(M y-n_{\Gamma}-\mu_{L}(\omega)\right)^{2}}{2 \sigma_{L}^{2}(\omega)}\right]
$$

provided

$$
\left(M y-n_{\Gamma}-\mu_{L}(\omega)\right)^{2} \leq C \sigma_{L}^{2}(\omega)
$$

where

$$
\mu_{L}(\omega)=\sum_{x \in \Lambda \backslash \Gamma} p_{x}^{\Gamma}
$$

and

$$
\sigma_{L}^{2}(\omega)=\sum_{x \in \Lambda \backslash \Gamma} p_{x}^{\Gamma}\left(1-p_{x}^{\Gamma}\right)
$$

Since $\sigma_{L}^{2}(\omega) \simeq C M y$ uniformly in $\omega$, it is sufficient to obtain a uniform lower bound

$$
\mu_{\Lambda, \lambda, \omega}\left\{\left(M y-n_{\Gamma}-\mu_{L}(\omega)\right)^{2} \leq C y M\right\} \geq \frac{1}{2}
$$

for some fixed $C$. By our choice of $\lambda$

$$
E^{\mu_{\Lambda, \lambda, \omega}}\left\{n_{\Gamma}+\mu_{L}(\omega)\right\}=M y
$$

and by orthogonality

$$
E^{\mu_{\Lambda, \lambda, \omega}}\left\{\left(n_{\Gamma}+\mu_{L}(\omega)-M y\right)^{2}\right\} \leq E^{\mu_{\Lambda, \lambda, \omega}}\left\{M^{2}(\bar{\eta}-y)^{2}\right\} \leq C M y
$$

An application of Chbechev's inequality completes the proof of Lemma 10.1 for the case $1-L^{\varepsilon-d} \geq y \geq L^{\varepsilon-d}$.

Case 2. $y \leq L^{\varepsilon-d}$. [Case 3. $y \geq 1-L^{\varepsilon-d}$ is similar. ]

We will actually prove the much stronger assertion

$$
E^{\mu_{\Lambda, y, \omega}}\left[\eta_{0}-\eta_{e}\right] \equiv 0
$$


which is of course a purely combinatorial fact. It is convenient to normalize the energy $\mathbf{H}=\sum_{x \in \Lambda_{L}} H\left(\tau_{x} \eta\right)$ by assuming that the local function $H(\eta)$ is 0 for the empty configuration. For any boundary condition $\omega$ and particle density $y=N L^{-d}$, we define the canonical partition function

$$
Z_{x}^{\omega}=\sum_{\eta: \bar{\eta}=y} \eta(x) \exp [\mathbf{H}(\eta)]
$$

and prove that for large enough $L, Z_{0}^{\omega} \equiv Z_{e}^{\omega}$.

We shall construct a graph with vertices in $\Lambda_{L}$, connecting every site with a particle to all the sites in a cube of width $2 R+1$ centered at the site with the particle. Since the total number of particles is small, the cube will split up into several connected clusters. each cluster has a protective layer of empty sites preventing any site outside the union of all the clusters with interacting with any other site. Denoting the interior clusters by $C_{1}, \cdots, C_{q}$ and the clusters that interact with the boundary by $D_{1} \cdots, D_{p}$ the Hamiltonian takes the form

$$
\mathbf{H}(\eta)=\sum_{0 \leq i \leq q} H\left(C_{i}\right)+\sum_{0 \leq i \leq p} H_{\omega}\left(D_{i}\right)
$$

The clusters are actually slightly more complicated animals, because one has to keep in mind that inside each cluster there are some occupied sites and some empty sites. We shall fix the boundary clusters, but translate the interior clusters by arbitrary translations. For fixed $\mathcal{C}=C_{1}, \cdots, C_{q}, D_{1}, \cdots, D_{p}$ we define

$$
Z_{x}^{\mathcal{C}}=\sum_{x_{1}, \cdots, x_{q}: \Delta_{x}} \exp \left[\sum_{0 \leq i \leq q} H\left(\tau_{x_{i}} C_{i}\right)+\sum_{0 \leq i \leq p} H_{\omega}\left(D_{i}\right)\right]
$$

where $\Delta_{x}$ is the set of $\left(x_{1} \cdots, x_{q}\right)$ such that $\left\{\tau_{x_{i}} C_{i}, 1 \leq i \leq q\right\}$ and $\left\{D_{j}, 1 \leq j \leq p\right\}$ are all mutually disjoint and $x \in \cup_{1 \leq i \leq q} C_{i}$. It is clearly sufficient to prove that for large $L$, and for any $\mathcal{C}$ and boundary condition $\omega$,

$$
Z_{0}^{\mathcal{C}}=Z_{e}^{\mathcal{C}}
$$

Since the energy depends only on the class $\mathcal{C}$ it is sufficient to prove that

$$
\left|\Delta_{0}\right|=\left|\Delta_{e}\right|
$$

This will be carried out by careful counting, using the inclusion exclusion formula.

First, we note that the number of particles in any cluster must be at least a fixed fraction of its diameter. Since the total number of particles is of the order $L^{\varepsilon}$ with $\varepsilon<1$, we can never connect any boundary cluster to the sites 0 or $e$ by a chain of overlapping clusters that are translates of $C_{i}$. To simplify the notation let us think of a graph $G$ with $p+q$ vertices corresponding to $C_{i}$ and $D_{j}$. We will have bonds connecting two vertices that correspond to two different $C$ 's or between $C$-vertex and $D$-vertex but not between two $D$-vertices. A bond represents the intersection of the corresponding clusters after arbitrary translations of the $C$ 's. For each $z=\left(x_{1}, \cdots, x_{q}\right)$ we have the corresponding collection of bonds $\Gamma(z)$. If $z=\left(x_{1}, \cdots, x_{q}\right)$ is to be counted in $\Delta_{x}, \Gamma(z)$ must be empty and $x \in \cup_{1 \leq i \leq q} \tau_{x_{i}} C_{i}$. Therefore

$$
\left|\Delta_{x}\right|=\#\left\{z: \Gamma(z)=\emptyset, x \in \cup_{1 \leq i \leq q} \tau_{x_{i}} C_{i}\right\}=\sum_{1 \leq i \leq q} \#\left\{z: \Gamma(z)=\emptyset, x \in \tau_{x_{i}} C_{i}\right\}
$$


because $x$ cannot belong to different $C$ 's without creating an intersection. For each summand

$$
\left|\Delta_{x}^{i}\right|=\#\left\{z: \Gamma(z)=\emptyset, x \in \tau_{x_{i}} C_{i}\right\}
$$

by inclusion exclusion formula

$$
\begin{aligned}
\left|\Delta_{x}^{i}\right| & =\sum_{r}(-1)^{r} \sum_{\Gamma:|\Gamma|=r} \#\left\{z: \Gamma(z) \supset \Gamma, x \in \tau_{x_{i}} C_{i}\right\} \\
& =\sum_{r}(-1)^{r} \sum_{\Gamma:|\Gamma|=r}\left|\Delta_{x}^{i, \Gamma}\right|
\end{aligned}
$$

It is now a simple matter to complete the proof by establishing

$$
\left|\Delta_{0}^{i, \Gamma}\right|=\left|\Delta_{e}^{i, \Gamma}\right|
$$

for all $i$ and $\Gamma$. The bonds $\Gamma$ divide the graph $G$ into connected components and the vertex $i$ is in some component $G_{0}$. $G_{0}$ cannot contain any of the $D$ vertices due to the absence of long chains. If we shift by $e$ all the clusters that correspond to vertices in $G_{0}$, this establishes a one to one map between $\Delta_{0}^{i, \Gamma}$ and $\Delta_{e}^{i, \Gamma}$, and we are done.

\section{REFERENCES}

[DV] Donsker, M.D., AND VARADhAN, S. R. S., Large deviations from a hydrodynamic scaling limit, Comm. Pure Appl. Math. 42 , 243-270, (1989).

[EMY] Esposito, R., MARra, R., AND YAU, H.T., Diffusive limit of asymmetric simple exclusion, Rev. in Math. Physics 6, 1233-1267, (1994).

[GPV] Guo, M.G., PAPAnicolau, G.C., and VARAdhan, S. R. S., Nonlinear diffusion limit for a system with nearest neighbor interactions, Comm. Math. Phys. 118, 31-59, (1988).

[JY] Jensen, L., YAU, H.T., Hydrodynamic scaling limits of simple exclusion models, Park City/IAS Mathematics Series.

[KLS] Kats, S.,Lebowitz, J.L., AND SPOHn, H., Non equilibrium steady state of stochastic lattice gas models of fast ionic conductors. J. Stat Phys. 34, 497-537, (1984).

[LOY1] LANDIM, C., Olla, S., AND YAU, H.T., First order correction for the hydrodynamic limit of asymmetric simple exclusion processes in dimension $d \geq 3$, Commun. Pure. Appl. Math. Vol L, 0149-0203, (1997).

[LOY2] C. LANDim , S. Olla AND H. T. YAU, Some properties of the diffusion coefficient for asymmetric simple exclusion processes, Ann. Prob. 24, 1779-1808, (1996).

[LY] LU, S.L., AND YaU, H.T., Spectral gap and Logarithmic Sobolev Inequality for Kawasaki and Glauber dynamics, Commun. Math. Phys. 156, 399-433 (1993).

[MOS] Martinelli, F., Olivieri, E. And Schomann, R., For 2-D lattice spin systems Weak Mixing Implies Strong Mixing, Commun. Math. Phys. 165, 33-47 (1994).

[N] NeWman, C.: Private communication.

[OVY] Olla, S., Varadhan, S.R.S., AND YAU, H.-T.; Hydrodynamical limit for a Hamiltonian system with weak noise, Comm. Math. Phys., 155, 523-560, (1993).

[Q1] QUASTEL, J., Diffusion of colour in the simple exclusion process, Comm. Pure Appl. Math., 45, 321-379, (1992).

[Q2] QUASTEL, J., Large deviations from a hydrodynamic scaling limit for a nongradient system, Ann. Prob. 23 No. 2, 724-742 (1995).

[QRV] Rezakhanlou, F., Quastel, J., AND VARAdhan, S.R.S., Large Deviations for the symmetric simple exclusion process in dimension $d \geq 3$. (submitted to Probability Theory and Related Fields)

[QY1] QUASTEL, J., AND YAU, H.T., Bulk diffusion in a system with site disorder, in preperation.

[QY2] QUASTEL, J., AND YAU, H.T., Lattice gases, large deviations, and the incompressible Navier-Stokes equations, Preprint. 
[RS] Reed, M., Simon, B., Methods of Modern Mathematical Physics, Vol. I-IV, Academic Press (1972).

[R] Rezakhanlou, F., Hydrodynamic limit for a system with finite range interactions, Comm. Math. Phys. 129, 445-480 (1990).

[S] Spohn, H., Large Scale Dynamics of Interacting Particles, Springer-Verlag New York (1991).

[SY] SPOHN, H., AND YAU, H.T., Bulk diffusivity of lattice gases close to criticality, Jour. Stat. Phys., 79, 231-241, (1995).

[V1] VARAdhan, S.R.S., Nonlinear diffusion limit for a system with nearest neighbor interactions II, in Asymptotic Problems in Probability Theory : Stochastic Models and Diffusion on Fractals, edited by K. Elworthy and N. Ikeda, Pitman Research Notes in Mathematics 283, Wiley, (1994). 75-128

[V2] Varadhan, S. R. S. Scaling limits for interacting diffusions, Comm. Math. Phys. 135 313-353, (1991).

[V3] S. R. S. VARAdHAN, Regularity of self diffusion coefficient, "The Dynkin Festschrift, Markov processes and their applications", edited by M. Freidlin, Birkhäuser, (1994).

[Y1] YAU, H.T., Relative Entropy and Hydrodynamics of a Ginzburg-Landau Model, Lett. Math. Phys. 22, 63-80 (1991).

[Y2] YAU, H.T., Logarithmic Sobolev inequality for lattice gases with mixing conditions, Commun. Math. Phys, 181, 367-408, (1997). 